Supporting Information for

\title{
Switching an Artificial P450 Peroxygenase into Peroxidase via Mechanism-guided Protein Engineering
}

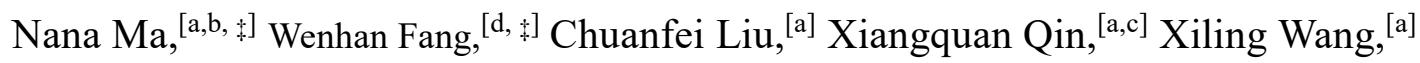 \\ Longyi Jin, ${ }^{[c]}$ Binju Wang, ${ }^{*[\mathrm{~d}]}$ and Zhiqi Cong ${ }^{*[a, b]}$ \\ ${ }^{[a]}$ CAS Key Laboratory of Biofuels and Shandong Provincial Key Laboratory of \\ Synthetic Biology, Qingdao Institute of Bioenergy and Bioprocess Technology, \\ Chinese Academy of Sciences. Qingdao, Shandong, 266101, China \\ ${ }^{[b]}$ University of Chinese Academy of Sciences. Beijing, 100049, China \\ ${ }^{[c]}$ Department of Chemistry, College of Science, Yanbian University. Yanji, Jilin, \\ 133002, China \\ ${ }^{[d]}$ Department of Chemistry, Xiamen University. Xiamen, Fujian, 361005, China
}

"These authors contributed equally.

*Correspondence to Zhiqi Cong, E-mail: congzq@qibebt.ac.cn

Binju Wang, E-mail: wangbinju2018@qibebt.ac.cn 


\section{Experimental Section}

\section{Instruments}

UV-visible spectra were recorded on UV vis absorption spectrophotometer (Shimadzu, UV1800). Gas chromatography (GC) analysis was performed on a Shimadzu GC-2010 plus gas chromatograph with a flame ionization detector (FID) and fitted with an AOC-20i auto sampler system by using a DB- 5 column (length: $30 \mathrm{~m}$, internal diameter: $0.25 \mathrm{~mm}$, film thickness: 1.0 $\mu \mathrm{m}$, Agilent, USA). Gas chromatography-mass spectroscopy (GC-MS) analysis was performed on an Agilent 7890 series gas chromatograph equipped with an Agilent 5975C mass selective detector (Agilent Technologies, Little Falls, DE, USA) and a HP-5MS capillary column $(30 \mathrm{~m} \times 0.25 \mathrm{~mm}$ $\times 0.25 \mu \mathrm{m}$, Agilent, USA).

\section{Materials}

All chemical reagents were purchased from commercial sources (e.g. Aldrich, TCI, Fluka, and Alladin) and used without further purification until otherwise noticed. Styrene was freshly prepared by passing through a short column packed with aluminum oxide. Dual-functional small molecule (DFSM), $N$-( $\omega$-imidazolyl)-hexanoyl-L-phenylalanine (Im-C6-Phe) was synthesized according to our previous report. ${ }^{[1]}$

\section{Expression and Purification of Cytochrome P450BM3 Enzymes}

The heme domain of Cytochrome P450BM3 and its variants were respectively cultured and purified according to previous report. ${ }^{[1]}$ Purified proteins were characterized by SDS pages. The formation of a ferrous $\mathrm{CO}$ complex was confirmed by UV/visible spectral change through the reduction of ferric heme of P450BM3 enzymes by addition of $\mathrm{Na}_{2} \mathrm{~S}_{2} \mathrm{O}_{4}$ in the presence of carbon monoxide $(\mathrm{CO}) \cdot{ }^{[2]}$

The concentrations of P450BM3 and its variants were measured by Hemochrome binding assay. ${ }^{[3]} \mathrm{A}$ general procedure is shown as below. A pyridine solution was made by combining pyridine $(1.75 \mathrm{~mL})$ and $1 \mathrm{M}$ aqueous of $\mathrm{NaOH}(0.75 \mathrm{~mL})$. The solution was mixed at room temperature then centrifuged for $30 \mathrm{~s}$ at $5000 \mathrm{rpm}$ to remove excess aqueous base. To a cuvette containing $0.75 \mathrm{~mL}$ of protein solution in phosphate buffer $(0.1 \mathrm{M}, \mathrm{pH} 8.0), 0.25 \mathrm{~mL}$ of the pyridine solution was added followed by $2 \mathrm{mg}$ of sodium dithionite. A UV/vis spectrum was recorded immediately. Hemoprotein concentration was determined from the absorbance of the hemochrome complex using extinction coefficients of $\varepsilon_{418 \mathrm{~nm}}=196 \mathrm{mM}^{-1} \mathrm{~cm}^{-1}$. Absorbance was assigned as the difference between the peak max at $418 \mathrm{~nm}$ and the baseline at $420 \mathrm{~nm}$ as determined by extrapolating from two points on either side of the hemochrome peak ( $390 \mathrm{~nm}$ and $450 \mathrm{~nm})$.

\section{Mutagenesis and Recombination}

All the mutations were made by PCR based site-directed mutagenesis and verified by DNA 
sequencing. The single mutants at the position of $\mathrm{F} 87$ were prepared according to previous report. ${ }^{[1]}$ The F87A mutants were then used as parent templates to prepare the double mutants containing the positions of F87 and W96 or F405 or M237 or Y160 or one tyrosine, respectively. Beneficial mutations selected from the prepared mutations were recombined. All primers used were as follows.

\begin{tabular}{|c|c|}
\hline primer & sequence \\
\hline F87A-F & 5'- GCGACAAGCTGGACGCATGAAAAAAATTG -3' \\
\hline F87A-R & 5'- GGTACAAGCTGGACGCATGAAAAAAATTGGAA -3' \\
\hline Y51I-F & 5'-ATTCTGTCGAGCCAGCGTCTAATTAAAG -3' \\
\hline Y51I-R & 5'- GCGCGTTACACGACCAGGC -3' \\
\hline Y115I-F & 5'-ATTCATGCGATGATGGTCGATATCG -3' \\
\hline Y115I-R & 5'- GCCTTTCATTGCCTGCTGACTGAAG -3' \\
\hline Y166I-F & 5'-ATTCGAGATCAGCCTCATCCATTTA -3' \\
\hline Y166I-R & 5'- AAAGCTGTTAAAGCGATAGTTAAAGCCGC -3' \\
\hline Y198I-F & 5'-ATTGATGAAAACAAGCGCCAGTTTC -3' \\
\hline Y198I-R & 5'- AGCTGGGTCGTCTGGATTTGC -3' \\
\hline Y256I-F & 5'- ATTTTCCTGGTGAAGAATCCACATG -3' \\
\hline Y256I-R & 5'- CAGCGCAAATGATAAAAGACCAC -3' \\
\hline Y278I-F & 5'- ATTCAGATCATCACGTTCTTAATTGCGGG -3' \\
\hline Y278I-R & 5'- GCGAATGTTCTCGTCATCAAGCG -3' \\
\hline Y305I-F & 5'- ATTAAGCAGGTCAAGCAGCTTAAATATGTCGG -3' \\
\hline Y305I-R & 5'- GCTTGGAACAGGATCTACTAGAACTCGTGC -3' \\
\hline Y313I-F & 5'-ATTGTCGGCATGGTCTTAAACGAAGC -3' \\
\hline Y313I-R & 5'- CTTAAGCTGCTTGACTTGTTTGTAGC -3' \\
\hline Y334I-F & 5'- ATTGCAAAAGAAGATACGGTGCTTGG -3' \\
\hline Y334I-R & 5'- TAGGGAAAACGCAGGAGCAGTTG -3' \\
\hline Y345I-F & 5'- ATTCCTTTAGAAAAAGGCGACGAAC -3' \\
\hline Y345I-R & 5'- TTCTCCTCCAAGCACCGTATCTTC -3' \\
\hline Y429I-F & 5'- ATTGAGCTGGACATCAAGGAAACTTTAACG -3' \\
\hline Y429I-R & 5'- GTTGGTATGATCTTCGAAGTCAAAGTC -3' \\
\hline Y160A-F & 5'- GCGCGCTTTAACAGCTTTTACCGAGATC -3' \\
\hline Y160I-F & 5'- ATTCGCTTTAACAGCTTTTACCGAG -3' \\
\hline Y160L-F & 5'- CTGCGCTTTAACAGCTTTTACCGAG -3' \\
\hline Y160V-F & 5'-GTGCGCTTTAACAGCTTTTACCGAG -3' \\
\hline Y160F-F & 5'- TTTCGCTTTAACAGCTTTTACCGAG -3' \\
\hline Y160G-F & 5'- GGTCGCTTTAACAGCTTTTACCGAG -3' \\
\hline Y160-R & 5'- GTTAAAGCCGCAAAGACCAATTG -3' \\
\hline M237A-F & 5'- GCGCTAAACGGAAAAGATCCAGAAAC -3' \\
\hline M237I-F & 5'- ATTCTAAACGGAAAAGATCCAGAAACGG -3' \\
\hline M237L-F & 5'-GTGCTAAACGGAAAAGATCCAGAAACG -3' \\
\hline M237V-F & 5'- CTGCTAAACGGAAAAGATCCAGAAACG -3' \\
\hline M237-R & 5'- ATGCGTTAATAAATCATCGCTTTGTTCACC -3' \\
\hline
\end{tabular}




\begin{tabular}{|c|l|}
\hline W96A-F & 5'- GCGAAGAAGGCGCATAATATCTTAC -3' \\
\hline W96I-F & 5'-ATTAAGAAGGCGCATAATATCTTACTTCCAAG -3' \\
\hline W96L-F & 5'- CTGAAGAAGGCGCATAATATCTTAC -3' \\
\hline W96V-F & 5'- GTGAAGAAGGCGCATAATATCTTAC -3' \\
\hline W96-R & 5'- GTTCTTCTCGTGCGTCCAGCTTG -3' \\
\hline F405A-F & 5'- GCGGCTCTTCATGAAGCAACG -3' \\
\hline F405I-F & 5'- ATTGCTCTTCATGAAGCAACGCTGG -3' \\
\hline F405L-F & 5'- CTGGCTCTTCATGAAGCAACG -3' \\
\hline F405V-F & 5'- GTGGCTCTTCATGAAGCAACGCTG -3' \\
\hline F405-R & 5'- CTGCTGACCGATACACGCAC -3' \\
\hline
\end{tabular}

\section{General procedure for guaiacol oxidation}

The reaction was carried out in $0.1 \mathrm{M}$ potassium phosphate buffer $(\mathrm{pH} 8.0$ or 7.0$)$ at $25^{\circ} \mathrm{C}$ in the presence of purified P450BM3 variants (20-500 nM), guaiacol (4-10 mM in $10 \mathrm{vol} . \% \mathrm{MeOH}$ in final reaction system), $N$-( $\omega$-imidazolyl)-hexanoyl- $L$-phenylalanine (Im-C6-Phe, 0.5-2 mM). Reactions were initiated by the addition of $20 \mathrm{mM}$ hydrogen peroxide. The total volume of reaction system was controlled within 1 or $2 \mathrm{~mL}$. The formation of tetra-guaiacol was confirmed by monitoring UV-visible absorption spectral change at the maximum absorption of $470 \mathrm{~nm}$, and total turnover numbers were determined related to the tetramer of guaiacol with a molar absorption coefficient of $\varepsilon_{470 \mathrm{~nm}}=2.66 \times 10^{4} \mathrm{M}^{-1} \mathrm{~cm}^{-1} \cdot{ }^{[4]}$

The product was further confirmed by GC-MS, and the product distribution was confirmed by GC. The sample was prepared as follows: when the formation of product almost didn't increase according to monitoring UV-visible absorption spectral change, the reaction was quenched and extracted with $1 \mathrm{~mL}$ of ethyl acetate, and the organic phase was separated and dried with sodium sulphate anhydrous. The sample was then analyzed on GC and GC-MS.

1) GC conditions: Splitting ratio: $1 / 9$, temperature program: injector $280{ }^{\circ} \mathrm{C}$, detector $280^{\circ} \mathrm{C}$, $80{ }^{\circ} \mathrm{C}$ oven for 1 minute, then $10{ }^{\circ} \mathrm{C} /$ min gradient to $140{ }^{\circ} \mathrm{C}, 60{ }^{\circ} \mathrm{C} / \mathrm{min}$ gradient to $280{ }^{\circ} \mathrm{C}$ and then $280^{\circ} \mathrm{C}$ for $15 \mathrm{~min}$ (total $29.0 \mathrm{~min}$ ).

2) GC-MS conditions: Carrier gas: $\mathrm{He}$, flow rate $1.0 \mathrm{~mL} / \mathrm{min}$, splitting ratio: $1 / 50$, inlet temperature: $300^{\circ} \mathrm{C}$, the oven temperature was initiated at $80^{\circ} \mathrm{C}$ for $1 \mathrm{~min}$, increased to $140^{\circ} \mathrm{C}$ at a rate of $10{ }^{\circ} \mathrm{C} / \mathrm{min}$, increased to $280^{\circ} \mathrm{C}$ at a rate of $60{ }^{\circ} \mathrm{C} / \mathrm{min}$, and then $280{ }^{\circ} \mathrm{C}$ for $8 \mathrm{~min}$, transfer line temperature: $280^{\circ} \mathrm{C}$, ion source temperature: $230^{\circ} \mathrm{C}$.

\section{Typical procedure for oxidation of 2,6-dimethoxyphenol (DMP)}

The reaction was carried out in $0.05 \mathrm{M}$ potassium phosphate buffer $(\mathrm{pH} 7.0)$ at $25^{\circ} \mathrm{C}$ in the presence of $20 \mathrm{nM}$ P450BM3, $4 \mathrm{mM}$ 2,6-dimethoxyphenol, $0.5 \mathrm{mM} \mathrm{N}$-( $\omega$-imidazolyl)-hexanoylL-phenylalanine (Im-C6-Phe). Reactions were initiated by the addition of $20 \mathrm{mM}$ hydrogen peroxide. The total volume of reaction system was controlled within $2 \mathrm{~mL}$. The formation of tetramethoxy-p-benzoquinone was confirmed by monitoring UV-visible absorption spectral change at the maximum absorption of $468 \mathrm{~nm}$, and total turnover numbers were determined related 
to the tetramer of tetramethoxy-p-benzoquinone with a molar absorption coefficient of $\varepsilon_{468 \mathrm{~nm}}=$ $4.96 \times 10^{4} \mathrm{M}^{-1} \mathrm{~cm}^{-1} \cdot{ }^{[5]}$ The product was further confirmed by GC-MS, and the sample was prepared with the same procedure of guaiacol described above.

GC-MS conditions: Carrier gas: He, flow rate $1.0 \mathrm{~mL} / \mathrm{min}$, splitting ratio: $1 / 50$, inlet temperature: $300{ }^{\circ} \mathrm{C}$, the oven temperature was initiated at $80^{\circ} \mathrm{C}$ for $1 \mathrm{~min}$, increased to $140^{\circ} \mathrm{C}$ at a rate of $10{ }^{\circ} \mathrm{C} / \mathrm{min}$, increased to $280{ }^{\circ} \mathrm{C}$ at a rate of $60{ }^{\circ} \mathrm{C} / \mathrm{min}$, and then $280{ }^{\circ} \mathrm{C}$ for $8 \mathrm{~min}$, transfer line temperature: $280{ }^{\circ} \mathrm{C}$, ion source temperature: $230^{\circ} \mathrm{C}$.

\section{Typical procedure for oxidation of $o$-phenylenediamine (OPD)}

The reaction was carried out in $0.05 \mathrm{M}$ potassium phosphate buffer $(\mathrm{pH} 7.0)$ at $25^{\circ} \mathrm{C}$ in the presence of $20 \mathrm{nM}$ P450BM3, $4 \mathrm{mM} o$-phenylenediamine, $0.5 \mathrm{mM} \mathrm{N}$-( $\omega$-imidazolyl)-hexanoyl- $L$ phenylalanine (Im-C6-Phe). Reactions were initiated by the addition of $20 \mathrm{mM}$ hydrogen peroxide. The total volume of reaction system was controlled within $2 \mathrm{~mL}$. The formation of 2,3diaminophenazine was confirmed by monitoring UV-visible absorption spectral change at the maximum absorption of $419 \mathrm{~nm}$, and total turnover numbers were determined related to 2,3diaminophenazine with a molar absorption coefficient of $\varepsilon_{419 \mathrm{~nm}}=1.54 \times 10^{4} \mathrm{M}^{-1} \mathrm{~cm}^{-1}$. The molar extinction coefficient of the product is determined by the standard curve (Figure S21). The product was further confirmed by GC-MS, and the sample was prepared with the same procedure of guaiacol described above.

GC-MS conditions: Carrier gas: He, flow rate $1.0 \mathrm{~mL} / \mathrm{min}$, splitting ratio: $1 / 50$, inlet temperature: $300{ }^{\circ} \mathrm{C}$, the oven temperature was initiated at $80{ }^{\circ} \mathrm{C}$ for $1 \mathrm{~min}$, increased to $140{ }^{\circ} \mathrm{C}$ at a rate of $10{ }^{\circ} \mathrm{C} / \mathrm{min}$, increased to $280{ }^{\circ} \mathrm{C}$ at a rate of $60{ }^{\circ} \mathrm{C} / \mathrm{min}$, and then $280{ }^{\circ} \mathrm{C}$ for $8 \mathrm{~min}$, transfer line temperature: $280{ }^{\circ} \mathrm{C}$, ion source temperature: $230{ }^{\circ} \mathrm{C}$.

\section{Typical procedure for oxidation of $\boldsymbol{p}$-phenylenediamine (PPD)}

The reaction was carried out in $0.05 \mathrm{M}$ potassium phosphate buffer $(\mathrm{pH} 7.0)$ at $25^{\circ} \mathrm{C}$ in the presence of $10 \mathrm{nM}$ P450BM3, $4 \mathrm{mM} p$-phenylenediamine, $0.5 \mathrm{mM} \mathrm{N}$-( $\omega$-imidazolyl)-hexanoyl- $L$ phenylalanine (Im-C6-Phe). Reactions were initiated by the addition of $20 \mathrm{mM}$ hydrogen peroxide. The total volume of reaction system was controlled within $2 \mathrm{~mL}$. The formation of Bandrowski's base was confirmed by ESI-MS and UV-visible absorption spectra.

The formed Bandrowski's base was determined with a molar absorption coefficient of $\varepsilon_{500} \mathrm{~nm}$ $=1.109 \times 10^{4} \mathrm{M}^{-1} \mathrm{~cm}^{-1}$ at its maximum absorption of $500 \mathrm{~nm} \cdot{ }^{[6]}$ Total turnover numbers for enzymatic reactions were calculated according to the difference of absorption intensity at $500 \mathrm{~nm}$ that was obtained by subtracting the blank control experiment from the observed absorption intensity.

Preparative scale experiment of Bandrowski's base: in a $50 \mathrm{~mL}$ round-bottom flask, the $2 \mu \mathrm{M} \mathrm{F87A/Y160I/Y198I、40} \mathrm{mM} p$-phenylenediamine and $0.5 \mathrm{mM} \mathrm{Im}$-C6-Phe were added to 50 $\mathrm{mM} \mathrm{pH} 7.0$ phosphate buffer (the total volume was $10 \mathrm{~mL}$ ). Then, $40 \mathrm{mM} \mathrm{H}_{2} \mathrm{O}_{2}$ was added and the reaction mixture was stirred at room temperature under aerobic conditions. After $6 \mathrm{~h}$, the $\mathrm{pH}$ 
of the reaction mixture was adjusted into 8.0. The insoluble product was separated by filtration and washed by $\mathrm{pH} 8.0$ buffer and water, and then dried over anhydrous sodium sulfate. ( $21 \mathrm{mg}$, Yield $=48.5 \%$ ). The product was identified by ${ }^{1} \mathrm{H}-\mathrm{NMR}$ (Figure S25).

\section{The steady-state kinetics for $\mathrm{H}_{2} \mathrm{O}_{2}$-dependent substrate oxidation catalyzed by P450BM3 variants in the presence of Im-C6-Phe}

The initial rate of different substrate oxidation was measured in $50 \mathrm{mM}$ potassium phosphate buffer (pH 7.0) with $20 \mathrm{nM} \mathrm{P} 450 \mathrm{BM} 3$ variants, $0.5 \mathrm{mM} \mathrm{Im-C6-Phe,} 20 \mathrm{mM} \mathrm{H}_{2} \mathrm{O}_{2}$ and substrate concentrations varying between 0.05 and $10.0 \mathrm{mM}$ (Figure S25). The initial rate was determined from the curve slope of the absorbance verse time during the first $60 \mathrm{~s}$ after starting the reaction upon $\mathrm{H}_{2} \mathrm{O}_{2}$ addition (A470 for guaiacol, A468 for DMP, A419 for OPD, A500 for PPD). The initial reaction rates obtained were fitted to the Michaelis-Menten equation by non-linear least-squares method by using ORIGIN software to determine the apparent $k_{\text {cat }}$ and $K_{\mathrm{M}}$ values. For $p$ phenylenediamine, the concentration of P450BM3 variants was $10 \mathrm{nM}$, for guaiacol, the concentration of Im-C6-Phe was $2 \mathrm{mM}$.

\section{Kinetic analysis for the heme degradation of P450BM3 variants}

To a freshly prepared solution of P450BM3 $(2 \mu \mathrm{M})$ in $0.1 \mathrm{M}, \mathrm{pH} 8.0$ phosphate buffer in a 1 cm quartz cuvette at $25^{\circ} \mathrm{C}, \mathrm{H}_{2} \mathrm{O}_{2}(20 \mathrm{mM}$, dissolved in $\mathrm{pH} 8.0$ phosphate buffer) was added. The UV-visible absorption spectral change at $418 \mathrm{~nm}$ was monitored. The time-course of the absorbance was simulated by a curve-fit program in ORIGIN software least square curve-fit with the single exponential function, $\mathrm{Abs}=\mathrm{A}_{0}+\mathrm{A} \exp \left(-k_{\text {obs }} \mathrm{s}\right)$. For the degradation of P450BM3 $(2 \mu \mathrm{M})$ in the presence of $\mathrm{H}_{2} \mathrm{O}_{2}(20 \mathrm{mM})$ and styrene $(4 \mathrm{mM})$, was simulated by a curve-fit program in ORIGIN software least square curve-fit with the single exponential function, $\mathrm{Abs}=\mathrm{A}_{0}+\mathrm{Aexp}(-$ $\left.k_{\text {obs }} \mathrm{t}\right)$.

\section{System setup and MD simulations}

For the system setup in the absence of DFSM, the PDB code of $2 \mathrm{~J}_{1} \mathrm{M}^{7}$ was used for both the F87A and F87A/Y160I/Y198I/M237I BM3 variants in the absence of DFSM. For the system setup in the presence of DFSM, our recently characterized crystal structure of BM3 in complex with DFSM (PDB code: 7EGN) was utilized for both the F87A and F87A/Y160I/Y198I/M237I BM3 variants in the presence of DFSM. All MD simulations were carried out in the Cpd I state in the presence of the substrate guaiacol. Guaiacol was docked into the active site of P450-BM3 using AutoDock Vina too ${ }^{8}$ in Chimera, ${ }^{9}$ respectively. Missing hydrogen atoms were added by module leap of Amber $18 .{ }^{10}$ The force field for the Cpd I state was taken from the literature. ${ }^{11}$ The general AMBER force field (GAFF) ${ }^{12}$ was used for guaiacol, while the partial atomic charges were obtained from the bcc method. The parmchk utility from AMBER Tools was used to generate the missing parameters for the ligands. $14 \mathrm{Na}^{+}$ions were added into the protein surface to neutralize the total charges of the systems. Finally, the resulting systems 
were solvated in a rectangular box of TIP3 $\mathrm{P}^{13}$ waters extending up to minimum cutoff of $16 \AA$ from the protein boundary. The Amber ff14SB force field ${ }^{14}$ was employed for the protein in all the molecular dynamics (MD) simulations. The initial structures were fully minimized using combined steepest descent and conjugate gradient method. The systems were then gently annealed from 0 to $300 \mathrm{~K}$ under canonical ensemble for $0.05 \mathrm{~ns}$ with a weak restraint of $25 \mathrm{kcal} / \mathrm{mol} / \AA$. 1 ns of density equilibration was performed under isothermal-isobaric ensemble at target temperature of $300 \mathrm{~K}$ and the target pressure of $1.0 \mathrm{~atm}$ using Langevin-thermostat ${ }^{15}$ and Berendsen barostat ${ }^{16}$ with collision frequency of $0.002 \mathrm{~ns}$ and pressure-relaxation time of 0.001 ns. Further equilibration of the systems was allowed for 4 ns to get well settled temperature and pressure. After proper minimizations and equilibrations, a productive MD run of $200 \mathrm{~ns}$ was performed for all the complex systems. The MD simulations were performed with GPU version of Amber 18 package. ${ }^{10}$

\section{QM Calculations with Gaussian}

All QM model calculations were performed with the Gaussian 16 software. ${ }^{17}$ Density functional theory of B3LYP ${ }^{18-20}$ was used in this study. For geometry optimizations and frequency calculations, the basis set of def2svp ${ }^{21}$ was used for all atoms, denoted as B1. The energies were further refined with the larger basis set of def2tzvpp ${ }^{21}$ for all atoms, denoted as B2. All calculations were performed in conjunction with the SMD continuum solvation model. ${ }^{22}$ Harmonic frequency calculations were performed at the B3LYP/B1 level to estimate the zero-point energies, as well as the thermal and entropic corrections at $298 \mathrm{~K}$. The final energies are given as the sum of electronic energy at B3LYP/B2 and thermal correction to Gibbs free energy at B3LYP/B1.

\begin{tabular}{lrrr}
\multicolumn{4}{l}{ The Cartesian Coordinates of the QM Region for all comp } \\
RC \\
C & -4.24212500 & 1.31954100 & 0.48143000 \\
H & -4.76470300 & 2.01500500 & -0.17453500 \\
$\mathrm{~N}$ & -3.02519200 & 1.48197100 & 0.97741500 \\
$\mathrm{C}$ & -2.77114400 & 0.36536600 & 1.74069400 \\
$\mathrm{H}$ & -1.81846200 & 0.21620000 & 2.24235900 \\
$\mathrm{C}$ & -3.86324800 & -0.47108700 & 1.70419300 \\
$\mathrm{H}$ & -4.05567700 & -1.43990500 & 2.15879300 \\
$\mathrm{~N}$ & -4.79976900 & 0.15899100 & 0.91041200 \\
$\mathrm{C}$ & -6.10469300 & -0.37340500 & 0.51684100 \\
$\mathrm{H}$ & -6.74061300 & 0.48470500 & 0.25573200 \\
$\mathrm{H}$ & -6.54727700 & -0.85295000 & 1.40232300 \\
$\mathrm{C}$ & -6.02063300 & -1.35648400 & -0.64353000 \\
$\mathrm{H}$ & -7.02753700 & -1.73073000 & -0.88554200 \\
$\mathrm{H}$ & -5.38472100 & -2.21804200 & -0.38727300 \\
$\mathrm{C}$ & 0.27083100 & 3.49378200 & 1.32602600 \\
$\mathrm{O}$ & -0.36614600 & 2.60490300 & 2.13910800
\end{tabular}




\begin{tabular}{|c|c|c|c|}
\hline $\mathrm{C}$ & 0.31666200 & 2.11086200 & 3.28058300 \\
\hline $\mathrm{H}$ & 1.21452700 & 1.53730600 & 2.99911400 \\
\hline $\mathrm{H}$ & 0.60348500 & 2.93116200 & 3.96167000 \\
\hline $\mathrm{H}$ & -0.38530800 & 1.44255800 & 3.79639300 \\
\hline $\mathrm{C}$ & -0.50315000 & 4.00583200 & 0.24982400 \\
\hline $\mathrm{O}$ & -1.77402200 & 3.61489700 & 0.01872200 \\
\hline $\mathrm{H}$ & -2.08390100 & 2.78912200 & 0.52296700 \\
\hline $\mathrm{C}$ & 0.07045900 & 4.96456700 & -0.59404800 \\
\hline $\mathrm{H}$ & -0.54228400 & 5.34795600 & -1.41363700 \\
\hline $\mathrm{C}$ & 1.38581300 & 5.40588000 & -0.40547700 \\
\hline $\mathrm{H}$ & 1.81027300 & 6.15061300 & -1.08321200 \\
\hline $\mathrm{C}$ & 2.14843300 & 4.88007000 & 0.63730300 \\
\hline $\mathrm{H}$ & 3.18142300 & 5.20140100 & 0.78874100 \\
\hline $\mathrm{C}$ & 1.58928700 & 3.92679100 & 1.50214800 \\
\hline $\mathrm{H}$ & 2.19059300 & 3.52262900 & 2.31651000 \\
\hline $\mathrm{H}$ & -5.60777200 & -0.86971100 & -1.54091700 \\
\hline $\mathrm{C}$ & 1.29531800 & -3.83923100 & -2.16017800 \\
\hline $\mathrm{H}$ & 1.63683800 & -4.36845000 & -1.25329000 \\
\hline $\mathrm{H}$ & 0.19585000 & -3.85730800 & -2.17819800 \\
\hline $\mathrm{S}$ & 1.93550800 & -2.15006000 & -2.14499700 \\
\hline $\mathrm{Fe}$ & 0.85822900 & -0.92121900 & -0.19083600 \\
\hline $\mathrm{N}$ & -0.48246200 & -2.41926300 & -0.01654200 \\
\hline $\mathrm{N}$ & 2.25331400 & -2.08150500 & 0.70440000 \\
\hline $\mathrm{N}$ & 2.29899500 & 0.43682000 & -0.62179100 \\
\hline $\mathrm{N}$ & -0.42515800 & 0.07616200 & -1.39194100 \\
\hline $\mathrm{C}$ & -1.76824800 & -2.43488500 & -0.50132500 \\
\hline $\mathrm{C}$ & -2.45020700 & -3.63076800 & -0.06820000 \\
\hline $\mathrm{C}$ & -1.55915300 & -4.33768900 & 0.68847400 \\
\hline $\mathrm{C}$ & -0.33406700 & -3.57418600 & 0.70647200 \\
\hline $\mathrm{C}$ & 2.04250300 & -3.30030000 & 1.31109300 \\
\hline $\mathrm{C}$ & 3.25439400 & -3.75251700 & 1.95041600 \\
\hline $\mathrm{C}$ & 4.19728100 & -2.78745100 & 1.74149000 \\
\hline $\mathrm{C}$ & 3.56679100 & -1.74371100 & 0.97190000 \\
\hline $\mathrm{C}$ & 3.59664800 & 0.43542800 & -0.17625200 \\
\hline $\mathrm{C}$ & 4.27946700 & 1.62722900 & -0.61952600 \\
\hline $\mathrm{C}$ & 3.37534700 & 2.35119900 & -1.34276200 \\
\hline $\mathrm{C}$ & 2.14159200 & 1.60141900 & -1.33440400 \\
\hline $\mathrm{C}$ & -0.21938900 & 1.29617100 & -1.98134700 \\
\hline $\mathrm{C}$ & -1.41479900 & 1.73143900 & -2.66377000 \\
\hline $\mathrm{C}$ & -2.34678600 & 0.74913100 & -2.48487600 \\
\hline $\mathrm{C}$ & -1.71654700 & -0.27601900 & -1.68804100 \\
\hline $\mathrm{C}$ & -2.34590000 & -1.45108600 & -1.29227900 \\
\hline $\mathrm{H}$ & -3.37597900 & -1.60222100 & -1.61156600 \\
\hline $\mathrm{C}$ & 0.84028200 & -3.99540100 & 1.32648300 \\
\hline
\end{tabular}




$\begin{array}{lrrr}\mathrm{H} & 0.81609300 & -4.94765800 & 1.85840300 \\ \mathrm{C} & 4.19393000 & -0.57307000 & 0.57632200 \\ \mathrm{H} & 5.23714900 & -0.43657900 & 0.86533200 \\ \mathrm{C} & 0.97572500 & 2.01006400 & -1.96867600 \\ \mathrm{H} & 0.99774600 & 2.96395700 & -2.49497500 \\ \mathrm{O} & 0.37887300 & -0.17587000 & 1.17366500 \\ \mathrm{H} & 1.70300300 & -4.36922700 & -3.03503000 \\ \mathrm{H} & -3.48066500 & -3.87538300 & -0.32202000 \\ \mathrm{H} & -1.69427400 & -5.29533600 & 1.18916200 \\ \mathrm{H} & 3.34780300 & -4.69285500 & 2.49183800 \\ \mathrm{H} & 5.23440900 & -2.76300300 & 2.07293100 \\ \mathrm{H} & 5.32004200 & 1.85840900 & -0.39649600 \\ \mathrm{H} & 3.50633900 & 3.30999500 & -1.84068500 \\ \mathrm{H} & -1.50612300 & 2.67414800 & -3.20063400 \\ \mathrm{H} & -3.37549100 & 0.70483900 & -2.83920800\end{array}$

TS

$\begin{array}{lrrr}\mathrm{C} & -4.21708400 & 1.40491600 & 0.38876000 \\ \mathrm{H} & -4.73804000 & 2.11695000 & -0.24783800 \\ \mathrm{~N} & -2.98175500 & 1.54139800 & 0.85874200 \\ \mathrm{C} & -2.68970500 & 0.42902900 & 1.61286500 \\ \mathrm{H} & -1.71406800 & 0.28252300 & 2.06383200 \\ \mathrm{C} & -3.79132600 & -0.38708600 & 1.60097700 \\ \mathrm{H} & -3.97532900 & -1.35673000 & 2.05569700 \\ \mathrm{~N} & -4.74908900 & 0.25312400 & 0.83711600 \\ \mathrm{C} & -6.07149800 & -0.27748600 & 0.48917600 \\ \mathrm{H} & -6.69891300 & 0.58023900 & 0.20974500 \\ \mathrm{H} & -6.49670900 & -0.71748200 & 1.40260400 \\ \mathrm{C} & -6.01434000 & -1.30335300 & -0.63391700 \\ \mathrm{H} & -7.02990600 & -1.67235600 & -0.84434500 \\ \mathrm{H} & -5.38573200 & -2.16285900 & -0.35422100 \\ \mathrm{C} & 0.22497100 & 3.45117000 & 1.35741500 \\ \mathrm{O} & -0.43475900 & 2.57258500 & 2.17399600 \\ \mathrm{C} & 0.22952000 & 2.08723900 & 3.32698100 \\ \mathrm{H} & 1.12877100 & 1.50536000 & 3.06614400 \\ \mathrm{H} & 0.51353000 & 2.91158800 & 4.00538800 \\ \mathrm{H} & -0.48119000 & 1.42726400 & 3.84294200 \\ \mathrm{C} & -0.55618800 & 3.96082500 & 0.26586300 \\ \mathrm{O} & -1.79372900 & 3.57432000 & 0.05068300 \\ \mathrm{H} & -2.30910900 & 2.47168400 & 0.57837400 \\ \mathrm{C} & 0.06980600 & 4.91599400 & -0.56822700 \\ \mathrm{H} & -0.52259400 & 5.31169700 & -1.39816700 \\ \mathrm{C} & 1.38840700 & 5.33863000 & -0.36182300\end{array}$




\begin{tabular}{|c|c|c|c|}
\hline $\mathrm{H}$ & 1.83257900 & 6.07572800 & -1.03677800 \\
\hline $\mathrm{C}$ & 2.13411300 & 4.80825400 & 0.69133800 \\
\hline $\mathrm{H}$ & 3.16920000 & 5.11617400 & 0.85646100 \\
\hline $\mathrm{C}$ & 1.54387900 & 3.86460900 & 1.55194800 \\
\hline $\mathrm{H}$ & 2.12832400 & 3.45688700 & 2.37747200 \\
\hline $\mathrm{H}$ & -5.60994800 & -0.85532100 & -1.55480600 \\
\hline $\mathrm{C}$ & 1.28713900 & -3.84583700 & -2.14281100 \\
\hline $\mathrm{H}$ & 1.64596500 & -4.36295300 & -1.23569200 \\
\hline $\mathrm{H}$ & 0.18777500 & -3.87760100 & -2.14675800 \\
\hline $\mathrm{S}$ & 1.90775900 & -2.14829100 & -2.15489800 \\
\hline $\mathrm{Fe}$ & 0.86835000 & -0.92155900 & -0.18765300 \\
\hline $\mathrm{N}$ & -0.46658700 & -2.42297000 & 0.00861600 \\
\hline $\mathrm{N}$ & 2.27654900 & -2.07305000 & 0.69620700 \\
\hline $\mathrm{N}$ & 2.29712400 & 0.44044100 & -0.64072300 \\
\hline $\mathrm{N}$ & -0.43608800 & 0.07010200 & -1.37392300 \\
\hline $\mathrm{C}$ & -1.75226900 & -2.45309600 & -0.47564900 \\
\hline $\mathrm{C}$ & -2.42477200 & -3.64978000 & -0.03097300 \\
\hline $\mathrm{C}$ & -1.52804200 & -4.34253900 & 0.73262500 \\
\hline $\mathrm{C}$ & -0.30831400 & -3.57063900 & 0.74073600 \\
\hline $\mathrm{C}$ & 2.07451300 & -3.28578000 & 1.31722800 \\
\hline $\mathrm{C}$ & 3.29663800 & -3.73388200 & 1.94054000 \\
\hline $\mathrm{C}$ & 4.23723800 & -2.77284700 & 1.70546900 \\
\hline $\mathrm{C}$ & 3.59457000 & -1.73493000 & 0.93743800 \\
\hline $\mathrm{C}$ & 3.60394900 & 0.43897500 & -0.22171400 \\
\hline $\mathrm{C}$ & 4.27623300 & 1.63236100 & -0.67480600 \\
\hline $\mathrm{C}$ & 3.35537100 & 2.35985500 & -1.37386400 \\
\hline $\mathrm{C}$ & 2.12298300 & 1.60834600 & -1.34336200 \\
\hline $\mathrm{C}$ & -0.24633500 & 1.29458000 & -1.95847700 \\
\hline $\mathrm{C}$ & -1.44947400 & 1.72021100 & -2.63291600 \\
\hline $\mathrm{C}$ & -2.36869500 & 0.72453600 & -2.45834100 \\
\hline $\mathrm{C}$ & -1.72404700 & -0.29652600 & -1.66859100 \\
\hline $\mathrm{C}$ & -2.33943100 & -1.47872300 & -1.27152100 \\
\hline $\mathrm{H}$ & -3.36839700 & -1.64097300 & -1.58942800 \\
\hline $\mathrm{C}$ & 0.87310700 & -3.98159300 & 1.35488500 \\
\hline $\mathrm{H}$ & 0.85693100 & -4.92882400 & 1.89604400 \\
\hline $\mathrm{C}$ & 4.21538300 & -0.56783300 & 0.52198200 \\
\hline $\mathrm{H}$ & 5.26401500 & -0.43067700 & 0.79046700 \\
\hline $\mathrm{C}$ & 0.94413400 & 2.01747000 & -1.95420900 \\
\hline $\mathrm{H}$ & 0.95462700 & 2.97256600 & -2.47779100 \\
\hline $\mathrm{O}$ & 0.40696400 & -0.17210200 & 1.18300200 \\
\hline $\mathrm{H}$ & 1.68826900 & -4.38009000 & -3.01815900 \\
\hline $\mathrm{H}$ & -3.45342400 & -3.90500000 & -0.28191000 \\
\hline $\mathrm{H}$ & -1.65577300 & -5.29636000 & 1.24247700 \\
\hline U & 3.39798400 & -4.66940800 & 2.48883800 \\
\hline
\end{tabular}




$\begin{array}{rrrr}\mathrm{H} & 5.28018300 & -2.74710300 & 2.01807200 \\ \mathrm{H} & 5.32110400 & 1.86364900 & -0.47300100 \\ \mathrm{H} & 3.47501000 & 3.32066900 & -1.87059000 \\ \mathrm{H} & -1.55445300 & 2.66539300 & -3.16266700 \\ \mathrm{H} & -3.39822200 & 0.67025100 & -2.80893300\end{array}$

PC

C

$$
-4.27559200
$$

1.38329400

0.45680900

$\mathrm{H}$

$-4.82443000$

2.13331300

$-0.10771600$

$\mathrm{N}$

$-3.00481000$

1.47167100

0.84736500

C

$-2.65753400$

0.32787700

1.52918900

$\mathrm{H}$

$-1.63573100$

0.14237700

1.85089800

C

$-3.77236500$

$-0.46368200$

1.56101100

$\mathrm{H}$

$-3.93431800$

$-1.45479700$

1.97483000

$-4.77466200$

0.22086200

0.89611200

C

$-6.11422300$

$-0.30272800$

0.59782200

$-6.75614900$

0.56149700

0.37966100

$\mathrm{H}$

$-6.48793200$

$-0.77678200$

1.51629800

$\mathrm{C}$

$-6.09839100$

$-1.28805300$

$-0.56219300$

$-7.12051100$

$-1.65421600$

$-0.74284600$

$-5.45506300$

$-2.15291000$

$-0.33873100$

0.27953300

3.36920600

1.39896600

$-0.48222100$

2.58168200

2.15299400

C

0.05880600

1.99336500

3.33949200

0.88054400

1.30884800

3.08713400

$\mathrm{H}$

$\mathrm{H}$

0.40408500

2.77679100

4.03262300

$-0.76252500$

1.42796700

3.79567200

$-0.42408700$

3.97723000

0.25788400

$-1.62174400$

3.67789200

$-0.00211200$

$-2.38070500$

2.28330600

0.62597700

0.31727300

4.92681400

$-0.53849000$

$-0.21459200$

5.38574400

$-1.37449400$

1.63267100

5.21326700

$-0.26333500$

2.18454500

5.92311800

$-0.88323600$

2.29312500

4.56809600

0.81536500

3.34517600

4.78736000

1.00890800

1.62523800

3.66190900

1.64034000

2.15502000

3.19079100

2.46757400

$-5.73280400$

$-0.80536200$

$-1.48171500$

$\mathrm{H}$

1.80459600

$-3.74104600$

$-1.96552900$

2.64210200

$-3.84973700$

$-1.25672500$

$\mathrm{H}$

0.92464200

$-4.23922100$

$-1.52619900$

$\mathrm{S}$

1.46640400

$-1.99203700$

$-2.37127500$ 


\begin{tabular}{|c|c|c|c|}
\hline $\mathrm{Fe}$ & 0.82419600 & -0.90916400 & -0.30127100 \\
\hline $\mathrm{N}$ & -0.54102600 & -2.38226500 & -0.01979600 \\
\hline $\mathrm{N}$ & 2.20250200 & -2.00919600 & 0.68751300 \\
\hline $\mathrm{N}$ & 2.23486300 & 0.48477800 & -0.73754300 \\
\hline $\mathrm{N}$ & -0.51223500 & 0.11331000 & -1.44168000 \\
\hline $\mathrm{C}$ & -1.81132800 & -2.42633400 & -0.52674700 \\
\hline $\mathrm{C}$ & -2.49560700 & -3.60781000 & -0.04662500 \\
\hline $\mathrm{C}$ & -1.61787600 & -4.26541600 & 0.76839300 \\
\hline $\mathrm{C}$ & -0.39402500 & -3.49168100 & 0.76976900 \\
\hline $\mathrm{C}$ & 1.97844400 & -3.17020600 & 1.37959500 \\
\hline $\mathrm{C}$ & 3.20203600 & -3.61025400 & 2.01490900 \\
\hline $\mathrm{C}$ & 4.16377400 & -2.69710000 & 1.68807900 \\
\hline $\mathrm{C}$ & 3.52185900 & -1.69370600 & 0.86537500 \\
\hline $\mathrm{C}$ & 3.55258500 & 0.45033700 & -0.36690500 \\
\hline $\mathrm{C}$ & 4.23302100 & 1.63500500 & -0.84923900 \\
\hline $\mathrm{C}$ & 3.30009700 & 2.37946800 & -1.51265900 \\
\hline $\mathrm{C}$ & 2.05386000 & 1.64529200 & -1.43929200 \\
\hline $\mathrm{C}$ & -0.32629700 & 1.33569100 & -2.02703100 \\
\hline $\mathrm{C}$ & -1.53857200 & 1.75352500 & -2.69923800 \\
\hline $\mathrm{C}$ & -2.44856600 & 0.74979700 & -2.52592900 \\
\hline $\mathrm{C}$ & -1.79236400 & -0.27356000 & -1.73925400 \\
\hline $\mathrm{C}$ & -2.39795700 & -1.46058000 & -1.34107700 \\
\hline $\mathrm{H}$ & -3.42721100 & -1.62927800 & -1.65780700 \\
\hline $\mathrm{C}$ & 0.77012300 & -3.85972500 & 1.44095500 \\
\hline $\mathrm{H}$ & 0.74225000 & -4.77522300 & 2.03442400 \\
\hline $\mathrm{C}$ & 4.16460500 & -0.56028400 & 0.36842300 \\
\hline $\mathrm{H}$ & 5.22679100 & -0.44702900 & 0.59262700 \\
\hline $\mathrm{C}$ & 0.86412200 & 2.05928600 & -2.03312300 \\
\hline $\mathrm{H}$ & 0.87206700 & 3.00999500 & -2.56595800 \\
\hline $\mathrm{O}$ & 0.37476000 & -0.12395600 & 1.10068300 \\
\hline $\mathrm{H}$ & 2.07151500 & -4.28000400 & -2.89032700 \\
\hline $\mathrm{H}$ & -3.51991000 & -3.87409800 & -0.30523300 \\
\hline $\mathrm{H}$ & -1.76135800 & -5.19482100 & 1.31840400 \\
\hline $\mathrm{H}$ & 3.29237700 & -4.50962700 & 2.62306500 \\
\hline $\mathrm{H}$ & 5.21512200 & -2.68065400 & 1.97318600 \\
\hline $\mathrm{H}$ & 5.28848000 & 1.85005800 & -0.68612300 \\
\hline $\mathrm{H}$ & 3.42110000 & 3.33876800 & -2.01315300 \\
\hline $\mathrm{H}$ & -1.65466900 & 2.69806900 & -3.22889300 \\
\hline $\mathrm{H}$ & -3.47729200 & 0.68884500 & -2.87916400 \\
\hline
\end{tabular}

\section{References}

[1] N. Ma, Z. Chen, J. Chen, J. Chen, C. Wang, H. Zhou, L. Yao, O. Shoji, Y. Watanabe, Z. Cong, Dual-functional small molecules for generating an efficient cytochrome P450BM3 
peroxygenase. Angew. Chem. Int. Ed. 2018, 57, 7628-7633.

[2] T. Omura, R. Sato, The carbon monoxide/binding pigment of liver microsomes. I. evidence for its hemoprotein nature. J. Biol. Chem. 1964, 239, 2370-2378.

[3] Z. J. Wang, H. Renata, N. E. Peck, C. C. Farwell, P. S. Coelho, F. H. Arnold, Improved cyclopropanation activity of histidine/ligated cytochrome $\mathrm{P} 450$ enables the enantioselective formal synthesis of levomilnacipran. Angew. Chem. Int. Ed. 2014, 53, 6810-6813.

[4] G. D. DePillis, B. P. Sishta, A. G. Mauk, P. R. Ortiz de Montellano, Small substrates and cytochrome c are oxidized at different sites of cytochrome c peroxidase. J. Biol. Chem. 1991, 266, 19334.

[5] A. Vidal-Limón, Águila Sergio, M. Ayala, C. V. Batista, R. Vazquez-Duhalt. Peroxidase activity stabilization of cytochrome P450BM3 by rational analysis of intramolecular electron transfer. J. Inorg. Biochem. 2013, 122, 18-26.

[6] Y. Zhang, Y. R. F. Schmid, S. Luginbühl, Q. Wang, P. S. Dittrich, P. Walde, Spectrophotometric Quantification of Peroxidase with $p$-Phenylene-diamine for Analyzing Peroxidase Encapsulating Lipid Vesicles. Anal. Chem. 2017, 89, 5484-5493.

[7] Kuper J.; Wong T. S.; Roccatano D.; Wilmanns M.; Schwaneberg U. Understanding a mechanism of organic cosolvent inactivation in heme monooxygenase P450 BM-3. J. Am. Chem. Soc. 2007, 129, 5786-5787.

[8] Trott O., Olson A. J., AutoDock Vina: improving the speed and accuracy of docking with a new scoring function, efficient optimization, and multithreading. J. Comput. Chem. 2010, 31, 455461.

[9] Pettersen E. F.; Goddard T. D.; Huang C. C.; Couch G. S.; Green-blatt D. M.; Meng E. C.; Ferrin T. E. UCSF Chimera-a visualization system for exploratory research and analysis. $J$. Comput. Chem. 2004, 25, 1605-1612.

[10] Case, D. A.; I.Y. Ben-Shalom, I. Y.; S.R. Brozell, S. R.; Cerutti, D. S.; Cheatham, T. E.; Cruzeiro, III, V. W. D.; Darden, T. A.; Duke, R. E.; Ghoreishi, D.; Gilson, M. K.; Gohlke, H.; Goetz, A. W.; Greene, D.; Harris, R.; Homeyer, N.; Izadi, S.; Kovalenko, A.; Kurtzman, T.; Lee, T. S.; LeGrand, S.; Li, P.; Lin, C.; Liu, J.; Luchko, T.; Luo, R.; Mermelstein, D.J.; Merz, K. M.; Miao, Y.; Monard, G.; Nguyen, C.; Nguyen, H.; Omelyan, I.; Onufriev, A.; Pan, F.; Qi, R.; Roe, D. R.; Roitberg, A.; Sagui, C.; Schott-Verdugo, S.; Shen, J.; Simmerling, C. L.; Smith, J.; Salomon-Ferrer, R.; Swails, J.; Walker, R. C.; Wang, J.; Wei, H.; Wolf, R. M.; Wu, X.; Xiao, L.; York D. M.; Kollman, P. A. 2018, AMBER 2018, University of California, San Francisco.

[11] Oda, A.; Yamaotsu, N.; Hirono, S., New AMBER force field parameters of heme iron for cytochrome $\mathrm{P} 450 \mathrm{~s}$ determined by quantum chemical calculations of simplified models. J. Comput. Chem. 2005, 26, 818-826.

[12] Wang, J.; Wolf, R. M.; Caldwell, J. W.; Kollman, P. A.; Case, D. A., Development and testing of a general amber force field. J. Comput. Chem. 2004, 25, 1157-1174.

[13] Jorgensen, W. L.; Chandrasekhar, J.; Madura, J. D.; Impey, R. W.; Klein, M. L., Comparison of simple potential functions for simulating liquid water. J. Chem. Phys., 1983, 79, 926-935. 
[14] Maier, J. A.; Martinez, C.; Kasavajhala, K.; Wickstrom, L.; Hauser, K. E.; Simmerling, C., ff14SB: improving the accuracy of protein side chain and backbone parameters from ff99SB. $J$. Chem. Theor. Comput. 2015, 11, 3696-3713.

[15] Izaguirre, J. A.; Catarello, D. P.; Wozniak, J. M.; Skeel, R. D., Langevin stabilization of molecular dynamics. J. Chem. Phys. 2001, 114, 2090-2098.

[16] Berendsen, H. J. C.; Postma, J. P. M.; van Gunsteren,W. F.; DiNola, A.; Haak, J. R., Molecular dynamics with coupling to an external bath. J. Chem. Phys. 1984, 81, 3684-3690.

[17] Frisch, M. J.; Trucks, G. W.; Schlegel, H. B.; Scuseria, G. E.; Robb, M. A.; Cheeseman, J. R.; Scalmani, G.; Barone, V.; Petersson, G. A.; Nakatsuji, H.; Li, X.; Caricato, M.; Marenich, A. V.; Bloino, J.; Janesko, B. G.; Gomperts, R.; Mennucci, B.; Hratchian, H. P.; Ortiz, J. V.; Izmaylov, A. F.; Sonnenberg, J. L.; Williams; Ding, F.; Lipparini, F.; Egidi, F.; Goings, J.; Peng, B.; Petrone, A.; Henderson, T.; Ranasinghe, D.; Zakrzewski, V. G.; Gao, J.; Rega, N.; Zheng, G.; Liang, W.; Hada, M.; Ehara, M.; Toyota, K.; Fukuda, R.; Hasegawa, J.; Ishida, M.; Nakajima, T.; Honda, Y.; Kitao, O.; Nakai, H.; Vreven, T.; Throssell, K.; Montgomery Jr., J. A.; Peralta, J. E.; Ogliaro, F.; Bearpark, M. J.; Heyd, J. J.; Brothers, E. N.; Kudin, K. N.; Staroverov, V. N.; Keith, T. A.; Kobayashi, R.; Normand, J.; Raghavachari, K.; Rendell, A. P.; Burant, J. C.; Iyengar, S. S.; Tomasi, J.; Cossi, M.; Millam, J. M.; Klene, M.; Adamo, C.; Cammi, R.; Ochterski, J. W.; Martin, R. L.; Morokuma, K.; Farkas, O.; Foresman, J. B.; Fox, D. J., Gaussian 16 Rev. A.03. 2016: Wallingford, CT.

[18] Lee, C. T.; Yang, W. T.; Parr, R. G. DEVELOPMENT OF THE COLLE-SALVETTI CORRELATION-ENERGY FORMULA INTO A FUNCTIONAL OF THE ELECTRONDENSITY. Phys. Rev. B 1988, 37, 785-789.

[19] Becke, A. D. DENSITY-FUNCTIONAL THERMOCHEMISTRY .2. THE EFFECT OF THE PERDEW-WANG GENERALIZED-GRADIENT CORRELATION CORRECTION. J. Chem. Phys. 1992, 97, 9173-9177.

[20] Becke, A. D. DENSITY-FUNCTIONAL THERMOCHEMISTRY .3. THE ROLE OF EXACT EXCHANGE. J. Chem. Phys. 1993, 98, 5648-5652.

[21] Weigend, F.; Ahlrichs, R. Balanced basis sets of split valence, triple zeta valence and quadruple zeta valence quality for $\mathrm{H}$ to Rn: Design and assessment of accuracy. Phys. Chem. Chem. Phys. 2005, 7, 3297-3305.

[22] Marenich, A. V.; Cramer, C. J.; Truhlar, D. G. Universal Solvation Model Based on Solute Electron Density and on a Continuum Model of the Solvent Defined by the Bulk Dielectric Constant and Atomic Surface Tensions. J. Phys. Chem. B 2009, 113, 6378-6396. 
(A)



Lane M: molecular mass standards; Lane 1-6: F87A/Y160A, F87A/Y160I, F87A/Y160L, F87A/Y160V, F87A/Y160F, F87A/Y160G.

(B)
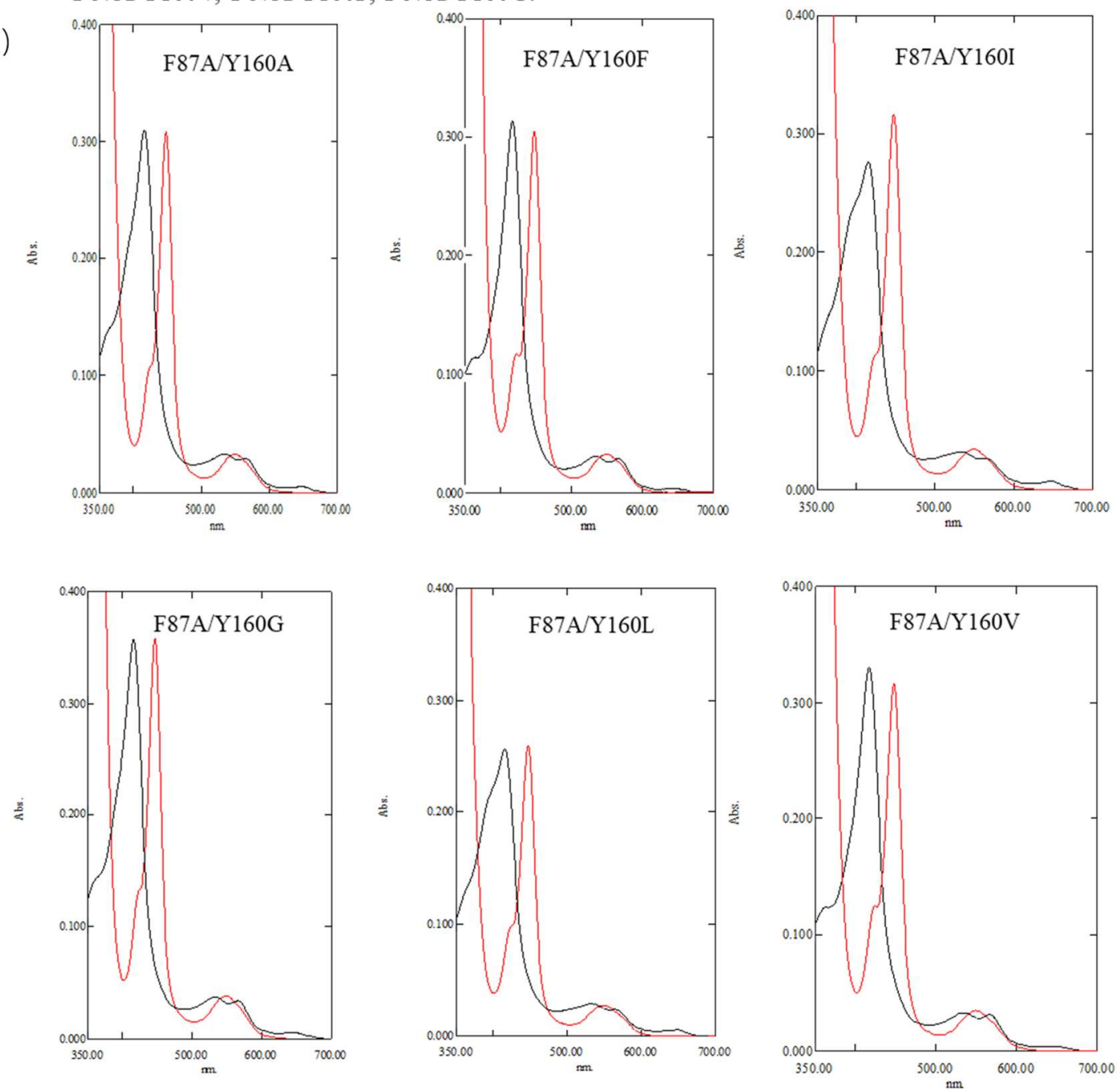

Figure S1 A) SDS page of the variants F87A/Y160X. B) UV/visible spectral changes of the variants $\mathrm{F} 87 \mathrm{~A} / \mathrm{Y} 160 \mathrm{X}$ (black line) upon addition of $\mathrm{Na}_{2} \mathrm{~S}_{2} \mathrm{O}_{4}$ (red line) for the formation of a ferrous $\mathrm{CO}$ complex through the reduction of ferric heme. 

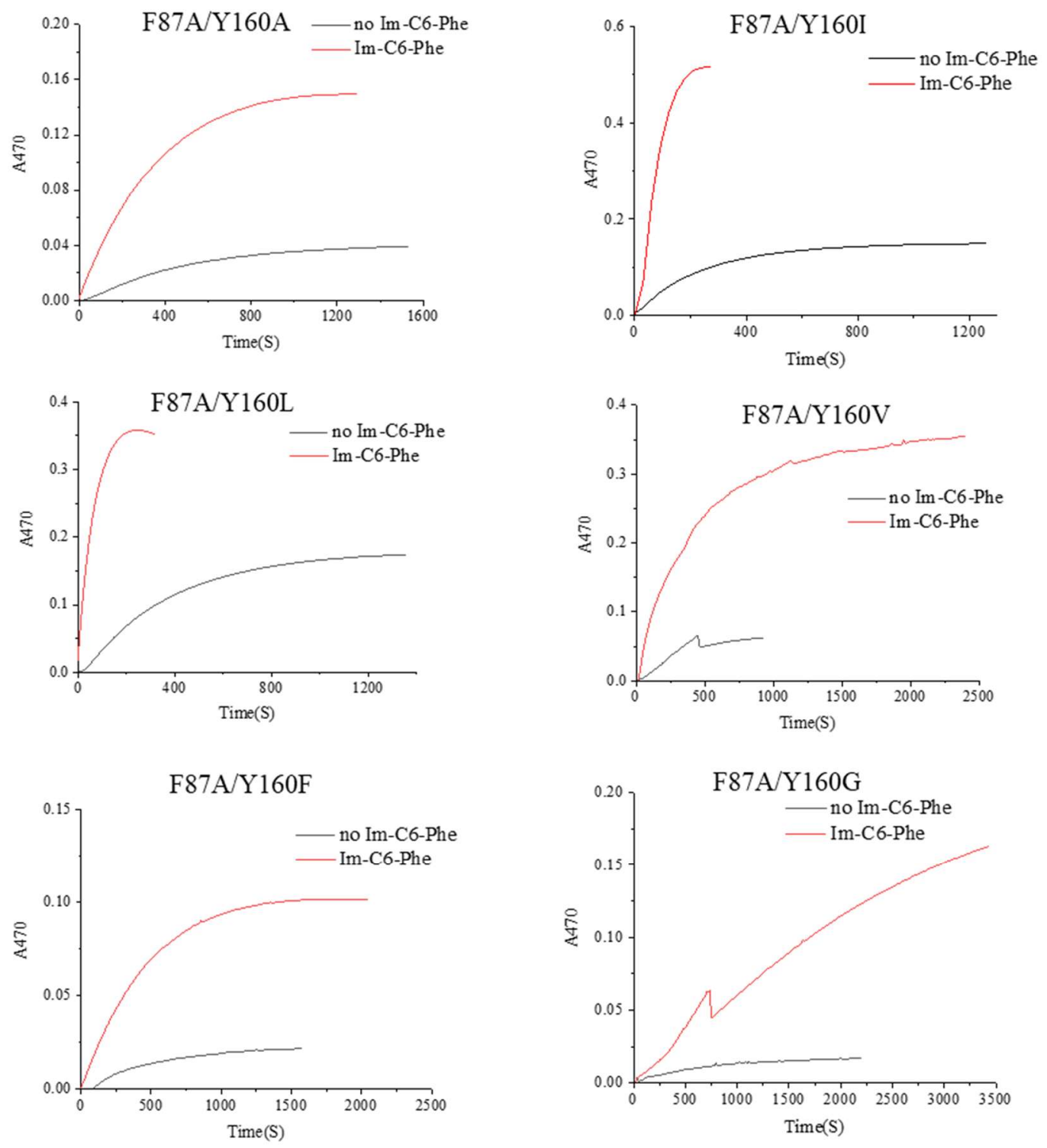

Figure S2 Time course of the absorbance at $470 \mathrm{~nm}$ for the oxidation of guaiacol catalyzed by the variants $\mathrm{F} 87 \mathrm{~A} / \mathrm{Y} 160 \mathrm{X}$ by addition of $\mathrm{H}_{2} \mathrm{O}_{2}(20 \mathrm{mM})$ in the absence (black line) / presence (red line) of Im-C6-Phe at $25^{\circ} \mathrm{C}$. 
(A)
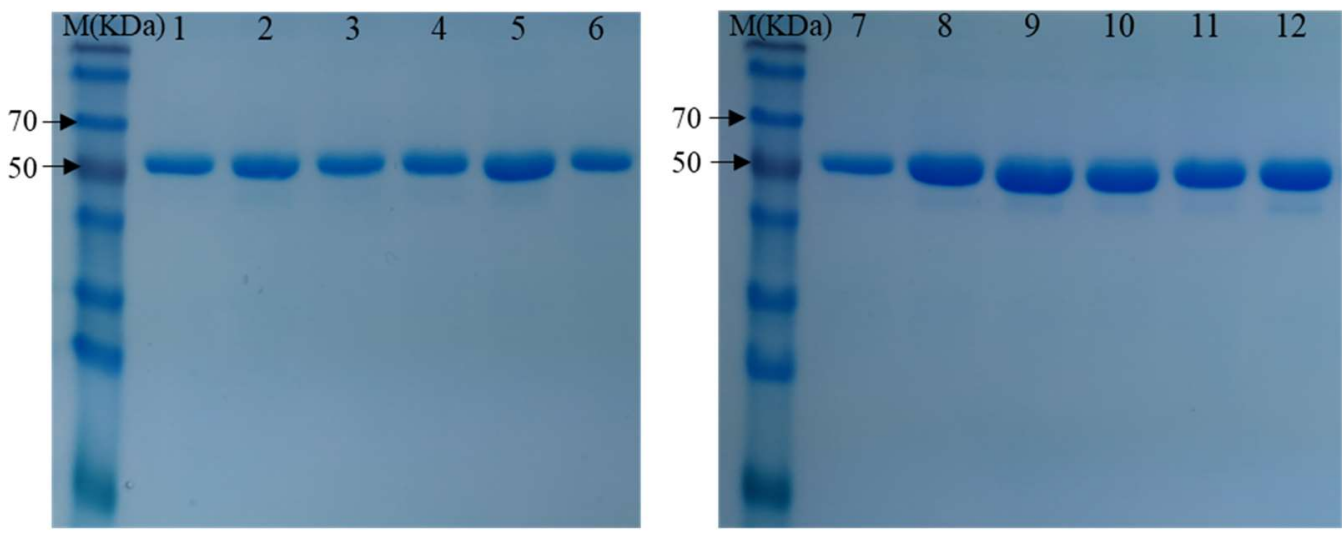

Lane M: molecular mass standards; Lane 1-12: F87A, F87A/Y51I, F87A/ Y115I, F87A/ Y166I, F87A/ Y198I, F87A/ Y256I, F87A/Y278I, F87A/Y305I, F87A/Y313I, F87A/Y334I, F87A/Y345I, F87A/Y429I.

(B)
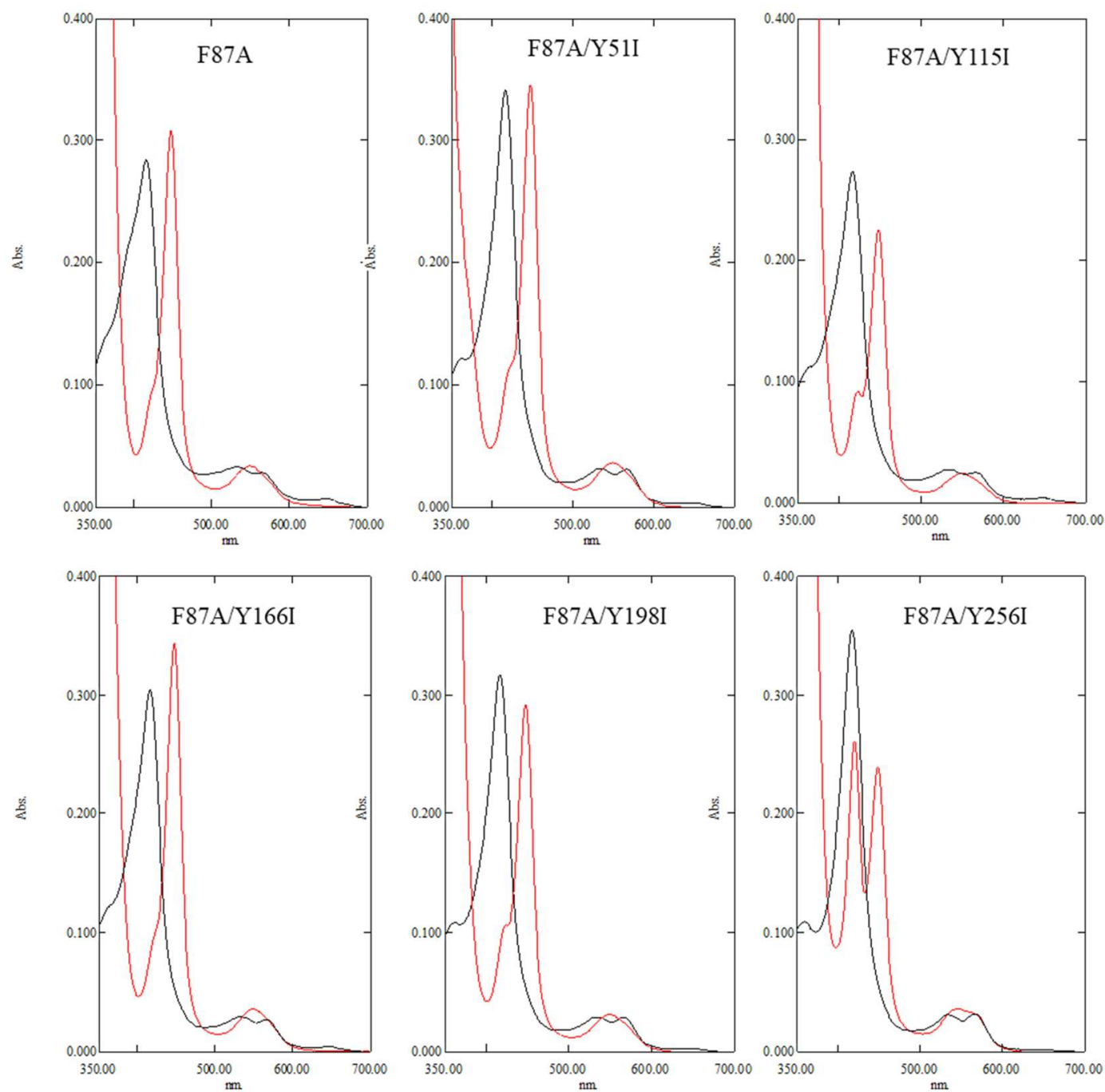

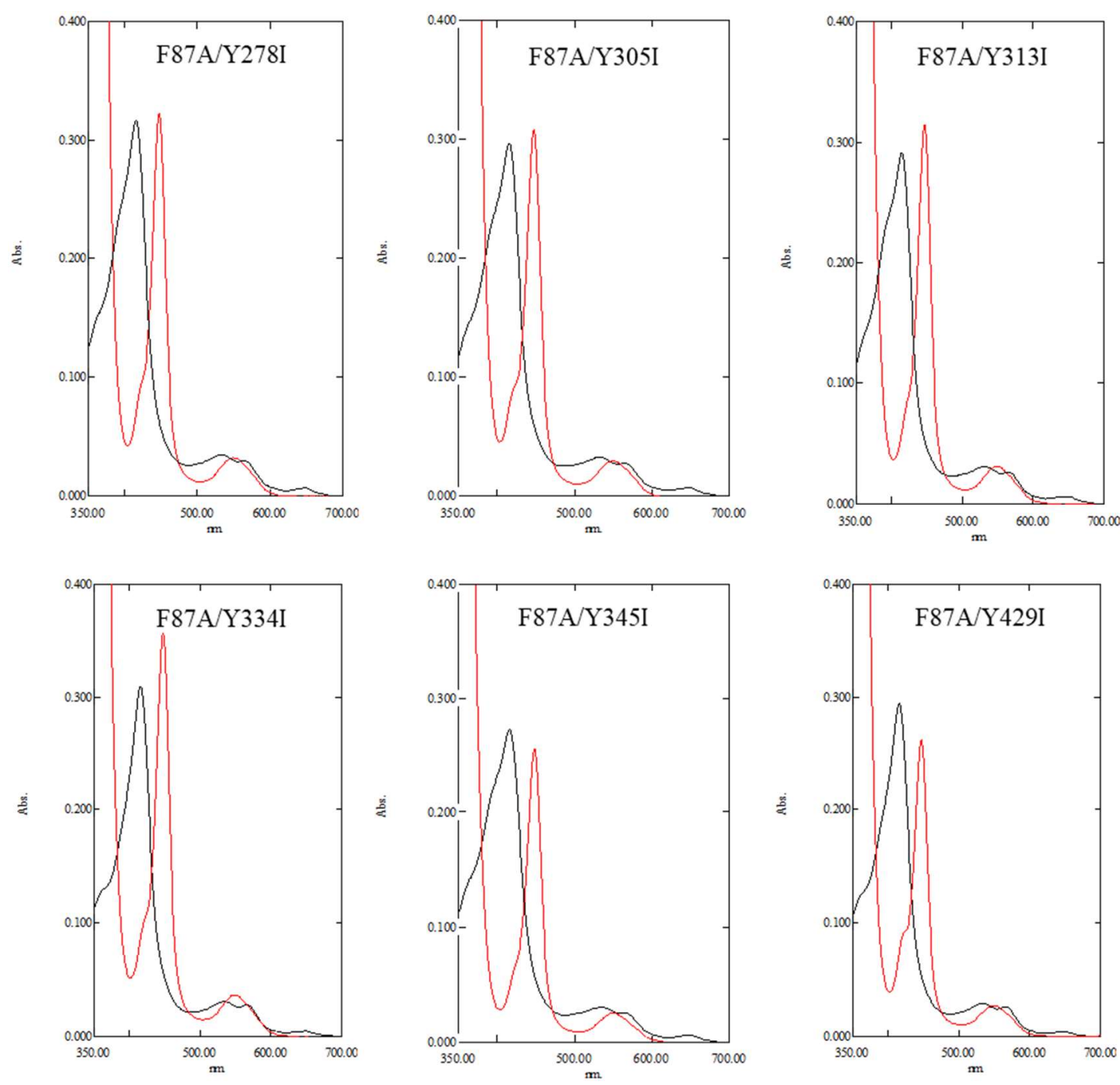

Figure S3 A) SDS page of P450BM3-F87A variants containing one tyrosine mutation. B) $\mathrm{UV} /$ visible spectral changes of $\mathrm{P} 450 \mathrm{BM} 3-\mathrm{F} 87 \mathrm{~A}$ variants containing one tyrosine mutation (black line) upon addition of $\mathrm{Na}_{2} \mathrm{~S}_{2} \mathrm{O}_{4}$ (red line) for the formation of a ferrous $\mathrm{CO}$ complex through the reduction of ferric heme. 

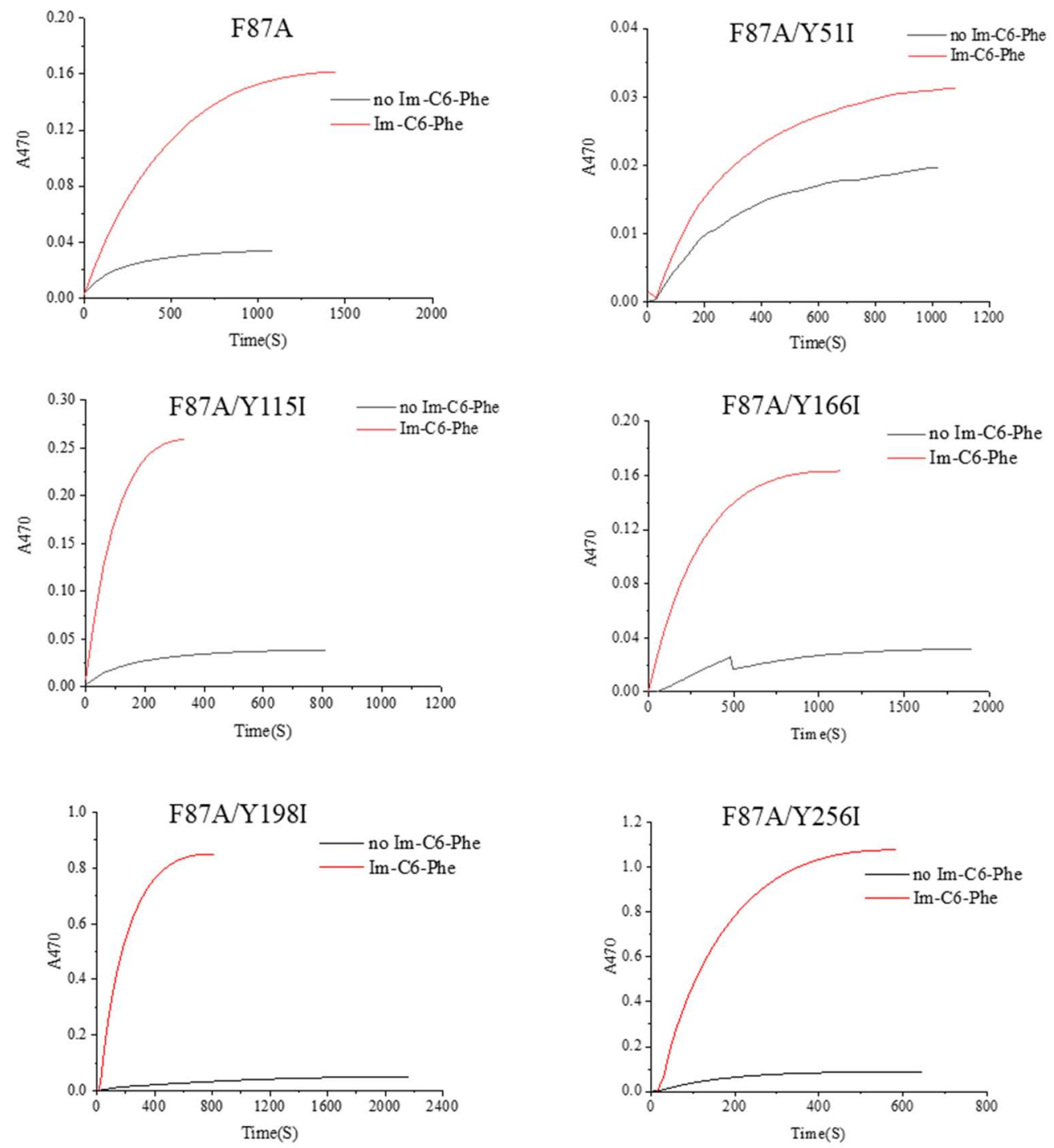

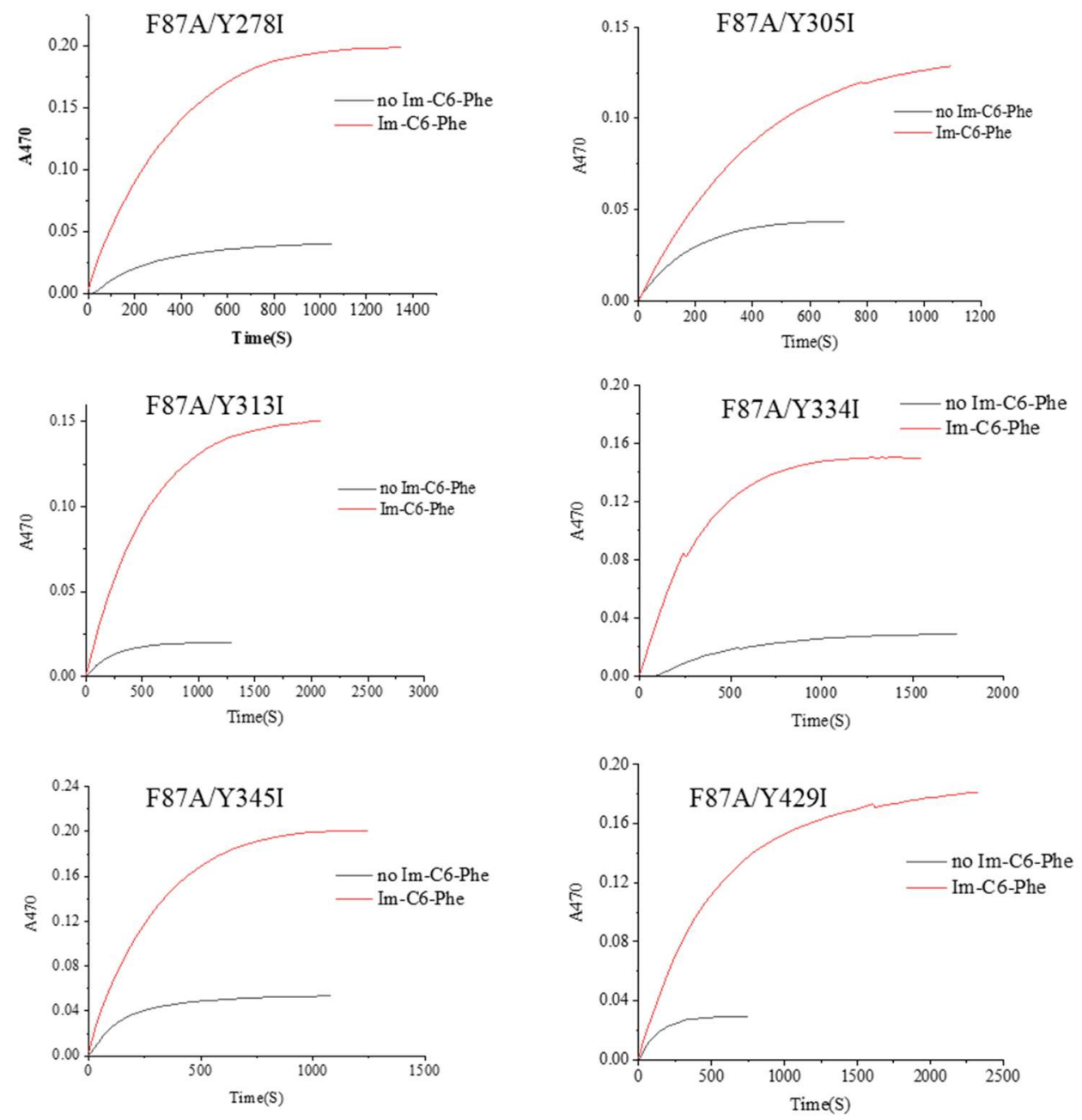

Figure S4 Time course of the absorbance at $470 \mathrm{~nm}$ for the oxidation of guaiacol catalyzed by P450BM3-F87A variants containing one tyrosine mutation by addition of $\mathrm{H}_{2} \mathrm{O}_{2}(20 \mathrm{mM})$ in the absence (black line) / presence (red line) of Im-C6-Phe at $25^{\circ} \mathrm{C}$. 
(A)

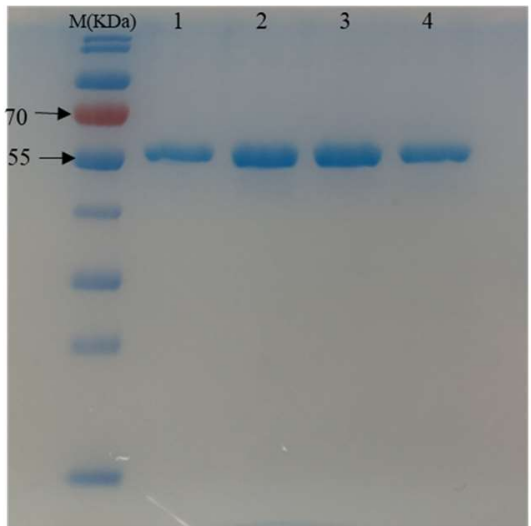

Lane M: molecular mass standards; Lane 1-4: F87A/Y160I/Y198I, F87A/Y160I/Y256I, F87A/Y198I/Y256I, F87A/Y160I/Y198I/Y256I.

(B)
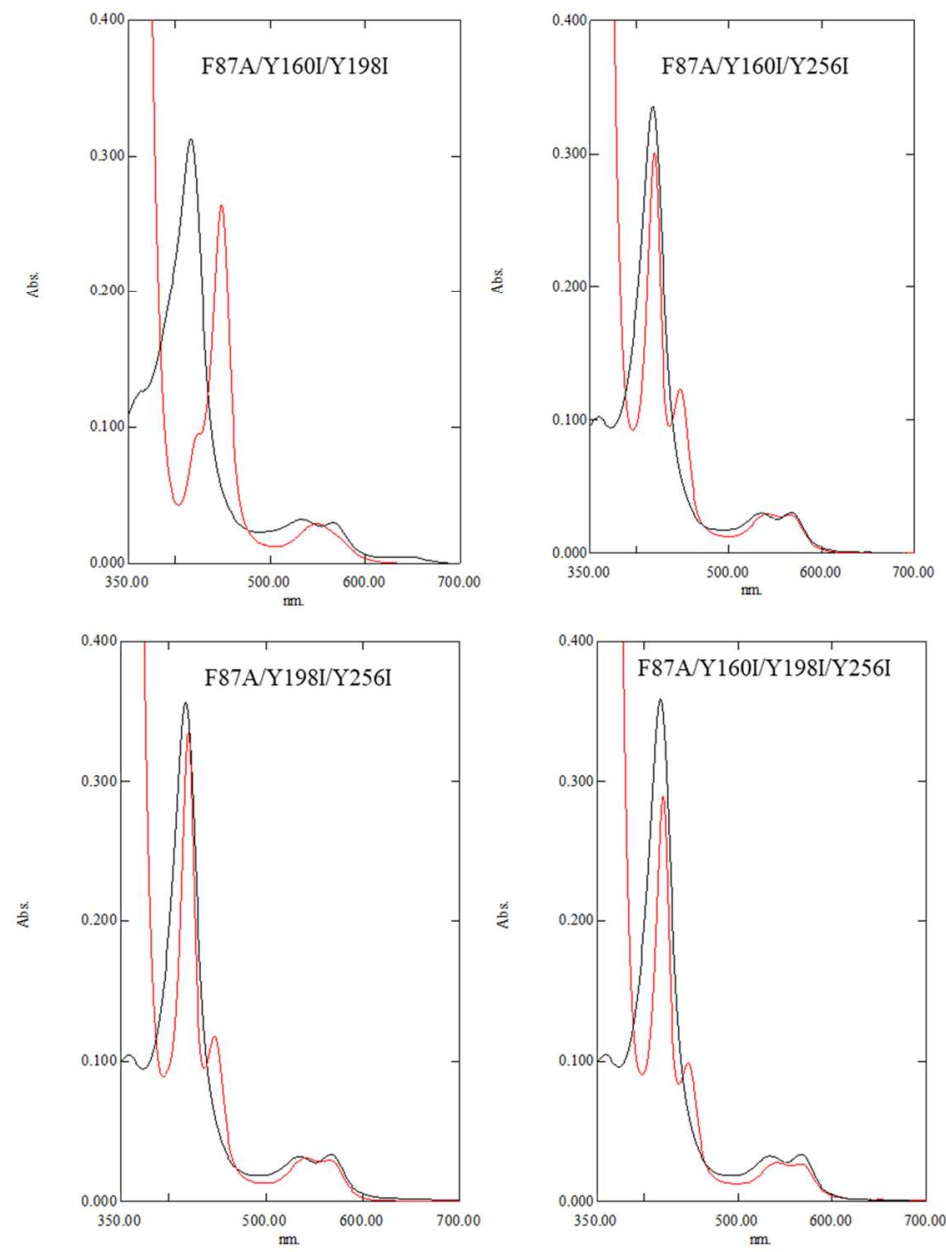

Figure S5 A) SDS page of P450BM3-F87A variants containing more than two tyrosine mutations. B) UV/visible spectral changes of P450BM3-F87A variants containing one tyrosine mutation (black line) upon addition of $\mathrm{Na}_{2} \mathrm{~S}_{2} \mathrm{O}_{4}$ (red line) for the formation of a ferrous $\mathrm{CO}$ complex through the reduction of ferric heme. 

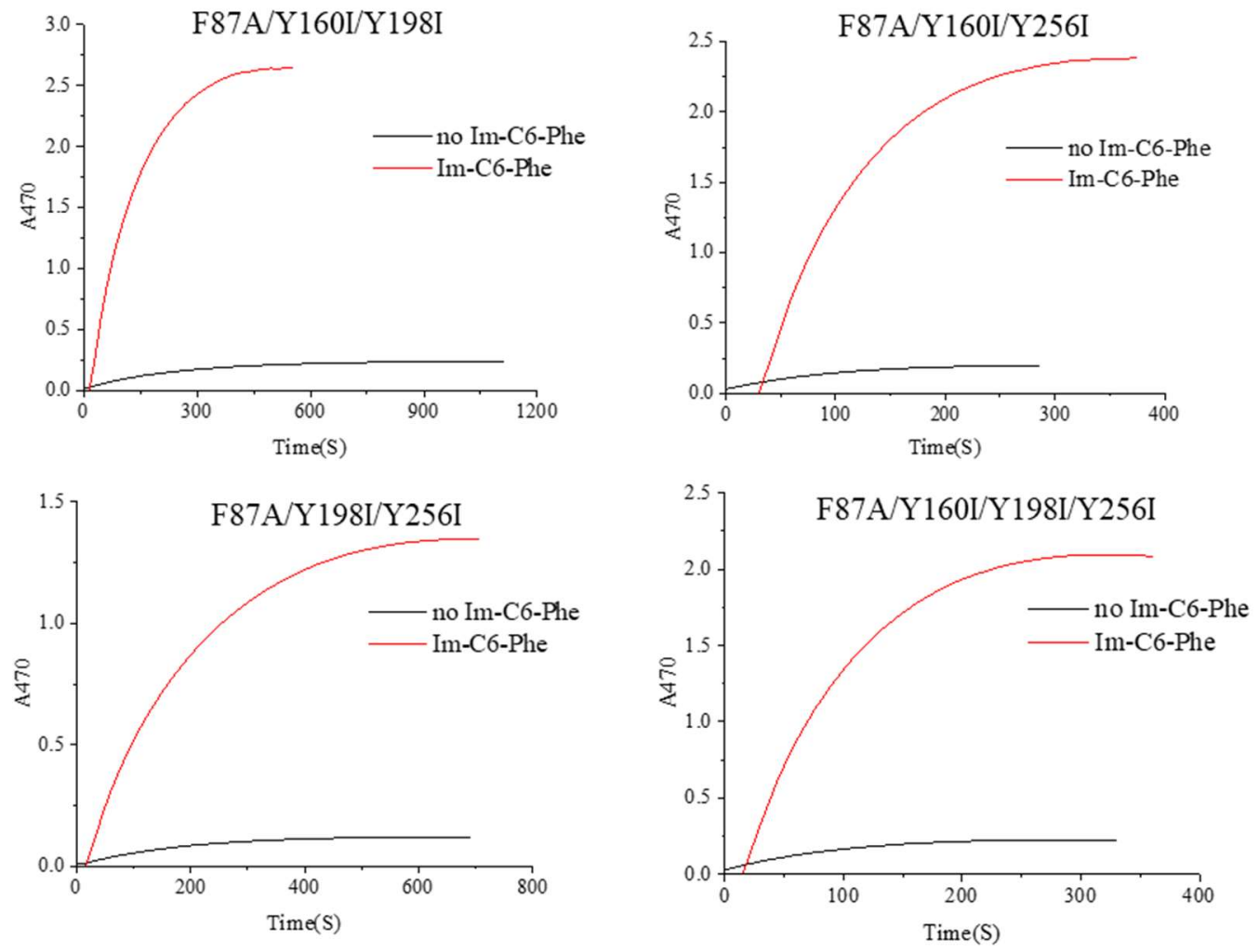

Figure S6 Time course of the absorbance at $470 \mathrm{~nm}$ for the oxidation of guaiacol catalyzed by P450BM3-F87A variants containing more than two tyrosine mutations by addition of $\mathrm{H}_{2} \mathrm{O}_{2}(20$ $\mathrm{mM}$ ) in the absence (black line) / presence (red line) of Im-C6-Phe at $25^{\circ} \mathrm{C}$. 



Figure 57 Optimizing the amount of $\mathrm{H}_{2} \mathrm{O}_{2}$ (A), guaiacol (B), Im-C6-Phe (C), and $\mathrm{MeOH}$ (D) used in the oxidation of guaiacol catalyzed by F87A/Y 160I/Y 198I in the presence of Im-C6-Phe in 0.1 $\mathrm{M} \mathrm{pH} 8.0$ phosphate buffer at $25^{\circ} \mathrm{C}$. The concentration of $\mathrm{F} 87 \mathrm{~A} / \mathrm{Y} 160 \mathrm{I} / \mathrm{Y} 198 \mathrm{I}$ is $0.5 \mu \mathrm{M}$ in the cases of A, B; the concentration of $\mathrm{F} 87 \mathrm{~A} / \mathrm{Y} 160 \mathrm{I} / \mathrm{Y} 198 \mathrm{I}$ is $0.1 \mu \mathrm{M}$ in the cases of $\mathrm{C}, \mathrm{D}$.

*The optimal reaction condition: the concentration of $\mathrm{H}_{2} \mathrm{O}_{2}$ is $20 \mathrm{mM}$, guaiacol is $10 \mathrm{mM}$, Im-C6Phe is $2 \mathrm{mM}$, methanol content is $10 \%$. Under these conditions, the TTN of guaiacol oxidation catalyzed by F87A/Y160I/Y198I is 2060. 
(A)

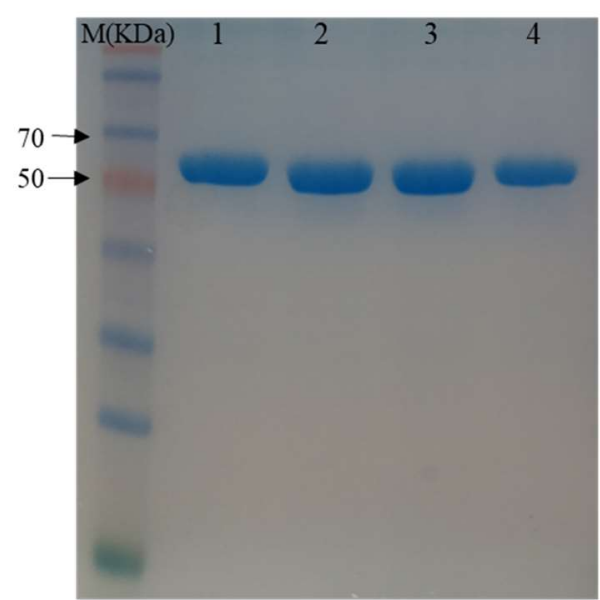

Lane M: molecular mass standards; Lane 1-4: F87A/W96A, F87A/ W96I, F87A/ W96L, F87A/ W96V.

(B)


Figure S8 A) SDS page of the variants F87A/W96X; B) UV/visible spectral changes of the variants $\mathrm{F} 87 \mathrm{~A} / \mathrm{W} 96 \mathrm{X}$ (black line) upon addition of $\mathrm{Na}_{2} \mathrm{~S}_{2} \mathrm{O}_{4}$ (red line) for the formation of a ferrous $\mathrm{CO}$ complex through the reduction of ferric heme. 
(A)



Lane M: molecular mass standards; Lane 1-4: F87A/M237A, F87A/ M237I, F87A/ M237L, F87A/ M237V.

(B)


Figure S9 A) SDS page of the variants F87A/M237X. B) UV/visible spectral changes of the variants F87A/ M237X (black line) upon addition of $\mathrm{Na}_{2} \mathrm{~S}_{2} \mathrm{O}_{4}$ (red line) for the formation of a ferrous $\mathrm{CO}$ complex through the reduction of ferric heme. 
(A)



Lane M: molecular mass standards; Lane 1-4: F87A/F405A, F87A/ F405I, F87A/ F405L, F87A/ F405V.

(B)
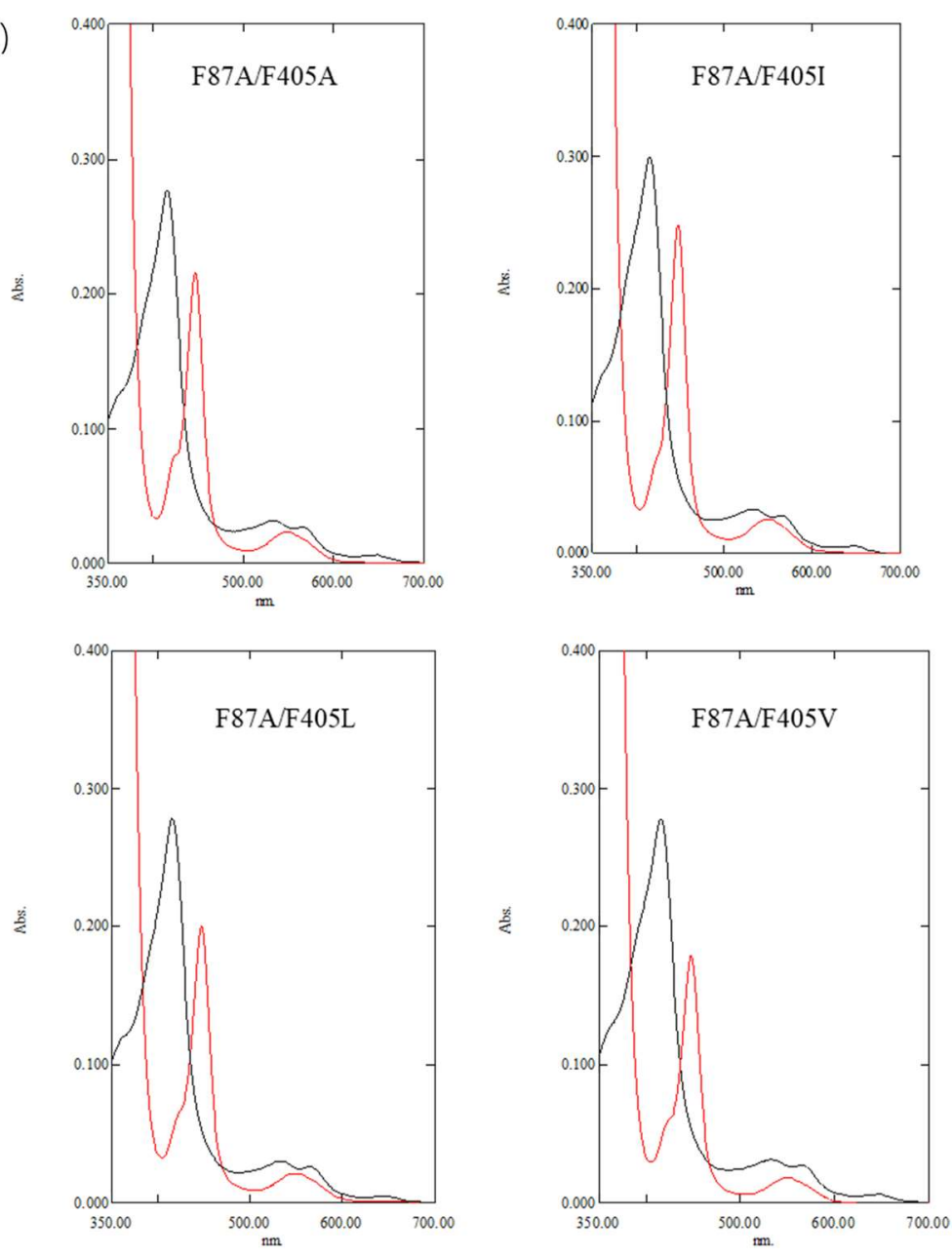

Figure S10 A) SDS page of the variants F87A/F405X. B) UV/visible spectral changes of the variants $\mathrm{F} 87 \mathrm{~A} / \mathrm{F} 405 \mathrm{X}$ (black line) upon addition of $\mathrm{Na}_{2} \mathrm{~S}_{2} \mathrm{O}_{4}$ (red line) for the formation of a ferrous $\mathrm{CO}$ complex through the reduction of ferric heme. 

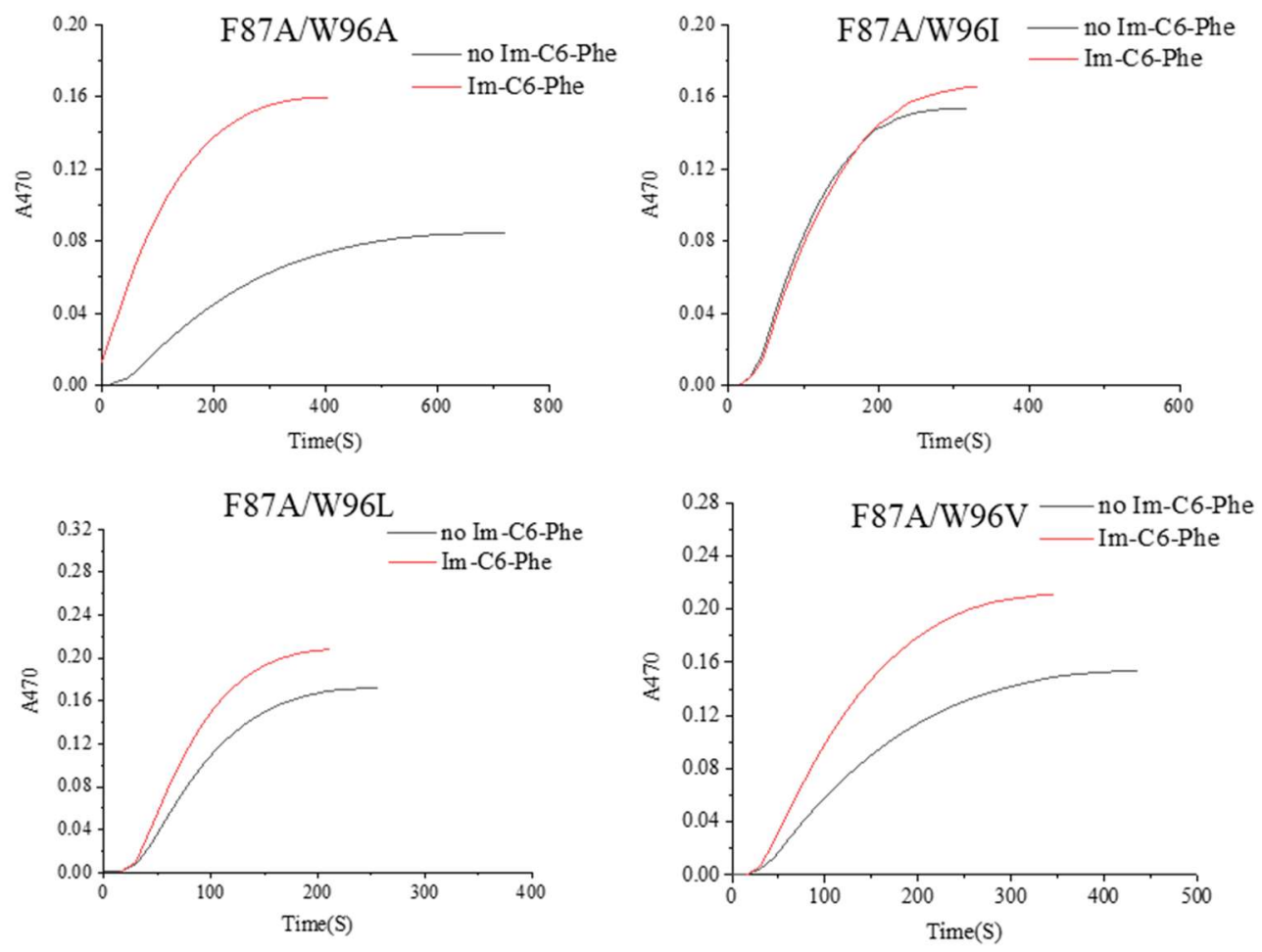

Figure S11 Time course of the absorbance at $470 \mathrm{~nm}$ for the oxidation of guaiacol catalyzed by the variants $\mathrm{F} 87 \mathrm{~A} / \mathrm{W} 96 \mathrm{X}$ by addition of $\mathrm{H}_{2} \mathrm{O}_{2}(20 \mathrm{mM})$ in the absence (black line) / presence (red line) of Im-C6-Phe at $25^{\circ} \mathrm{C}$.
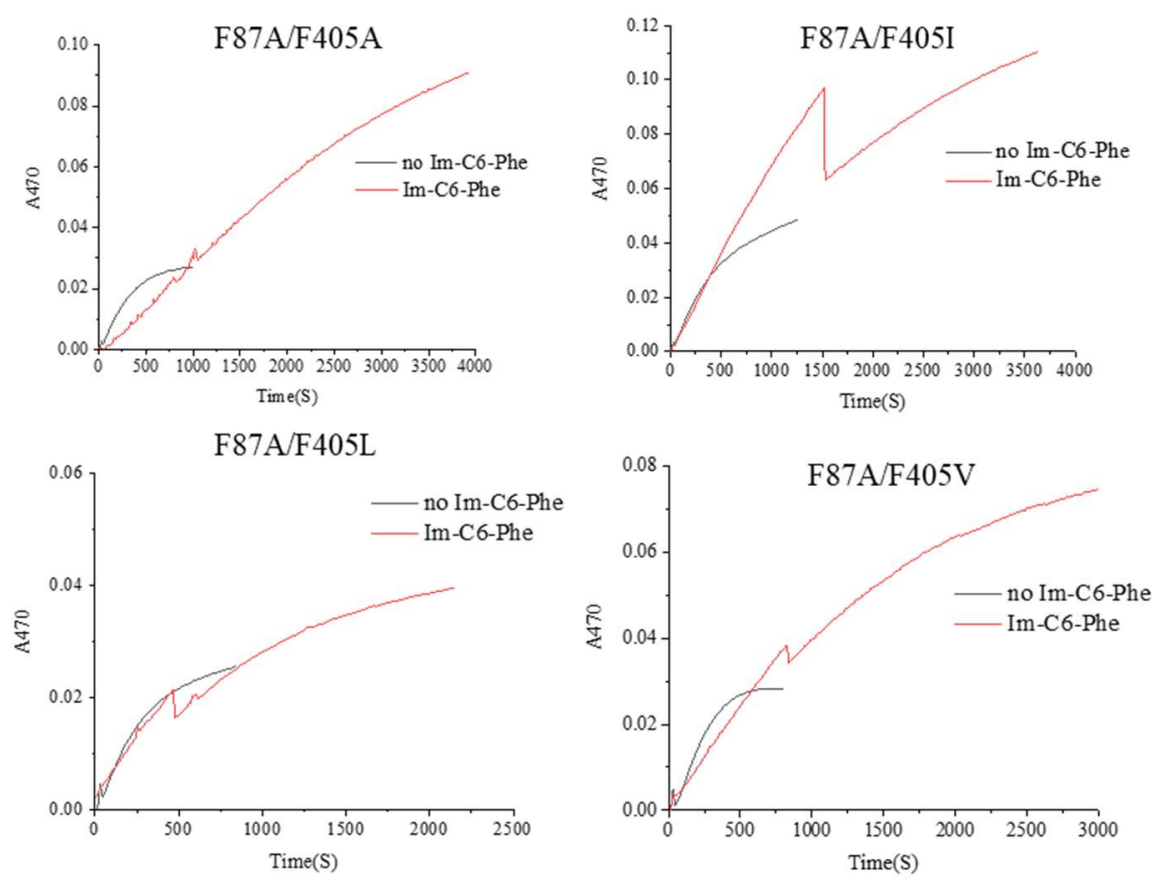

Figure S12 Time course of the absorbance at $470 \mathrm{~nm}$ for the oxidation of guaiacol catalyzed by the variants $\mathrm{F} 87 \mathrm{~A} / \mathrm{F} 405 \mathrm{X}$ by addition of $\mathrm{H}_{2} \mathrm{O}_{2}(20 \mathrm{mM})$ in the absence (black line) / presence (red line) of Im-C6-Phe at $25{ }^{\circ} \mathrm{C}$. 

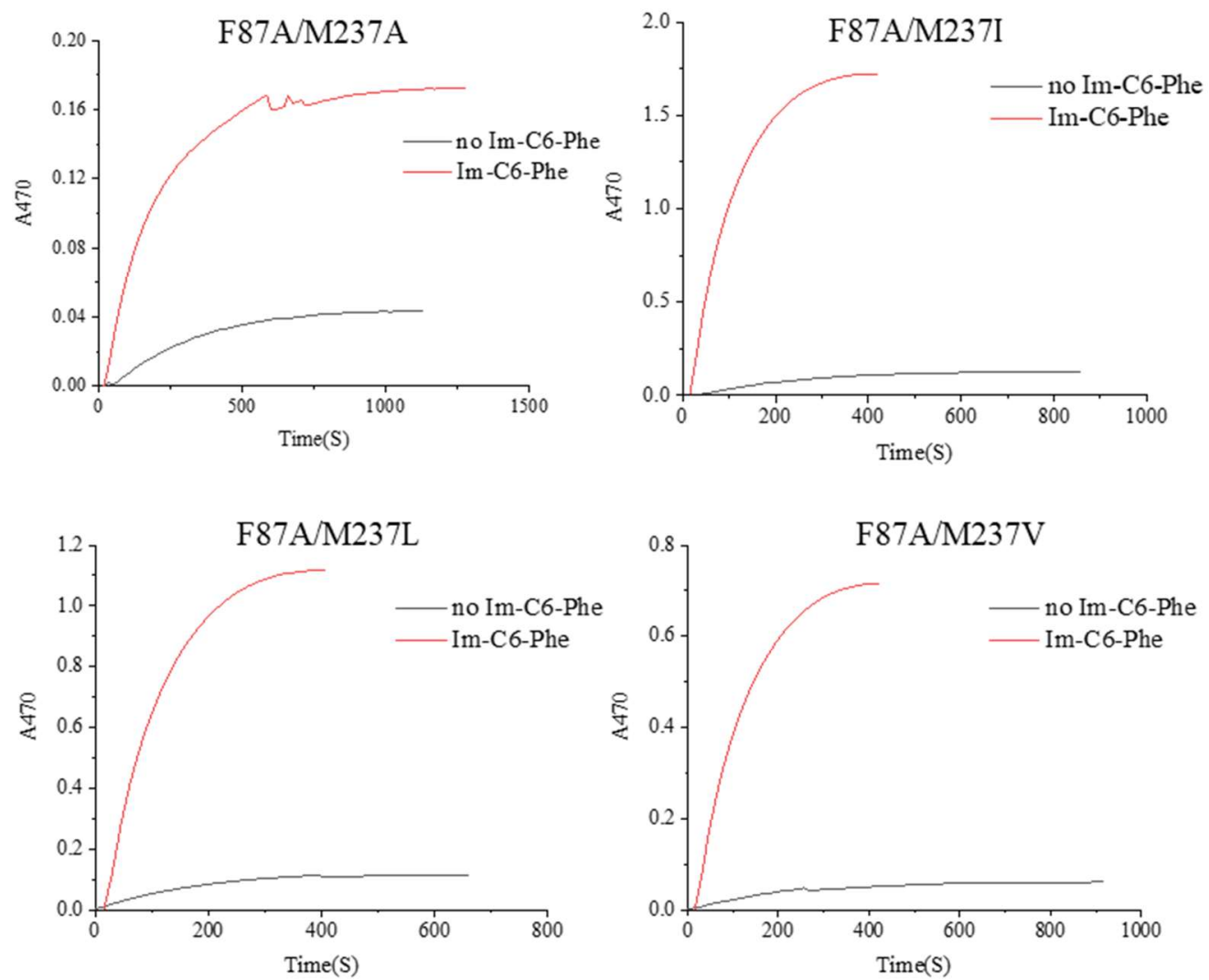

Figure S13 Time course of the absorbance at $470 \mathrm{~nm}$ for the oxidation of guaiacol catalyzed by the variants $\mathrm{F} 87 \mathrm{~A} / \mathrm{M} 237 \mathrm{X}$ by addition of $\mathrm{H}_{2} \mathrm{O}_{2}(20 \mathrm{mM})$ in the absence (black line) / presence (red line) of Im-C6-Phe at $25^{\circ} \mathrm{C}$. 
(A)

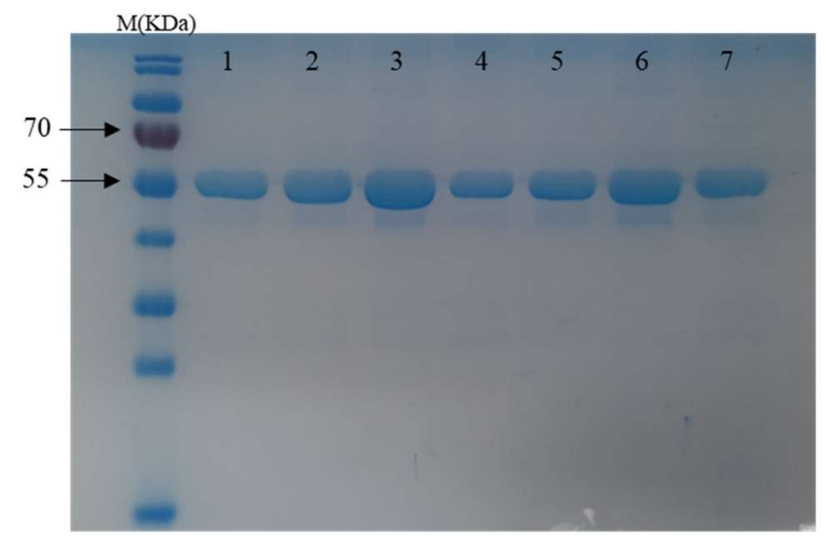

Lane M: molecular mass standards; Lane 1-7: F87A/Y160I/M237I, F87A/Y198I/M237I,

F87A/Y256I/M237I, F87A/Y160I/Y198I/M237I, F87A/Y160I/Y256I/M237I,

F87A/Y198I/Y256I/M237I, F87A/Y160I/Y198I/Y256I/M237I.

(B)
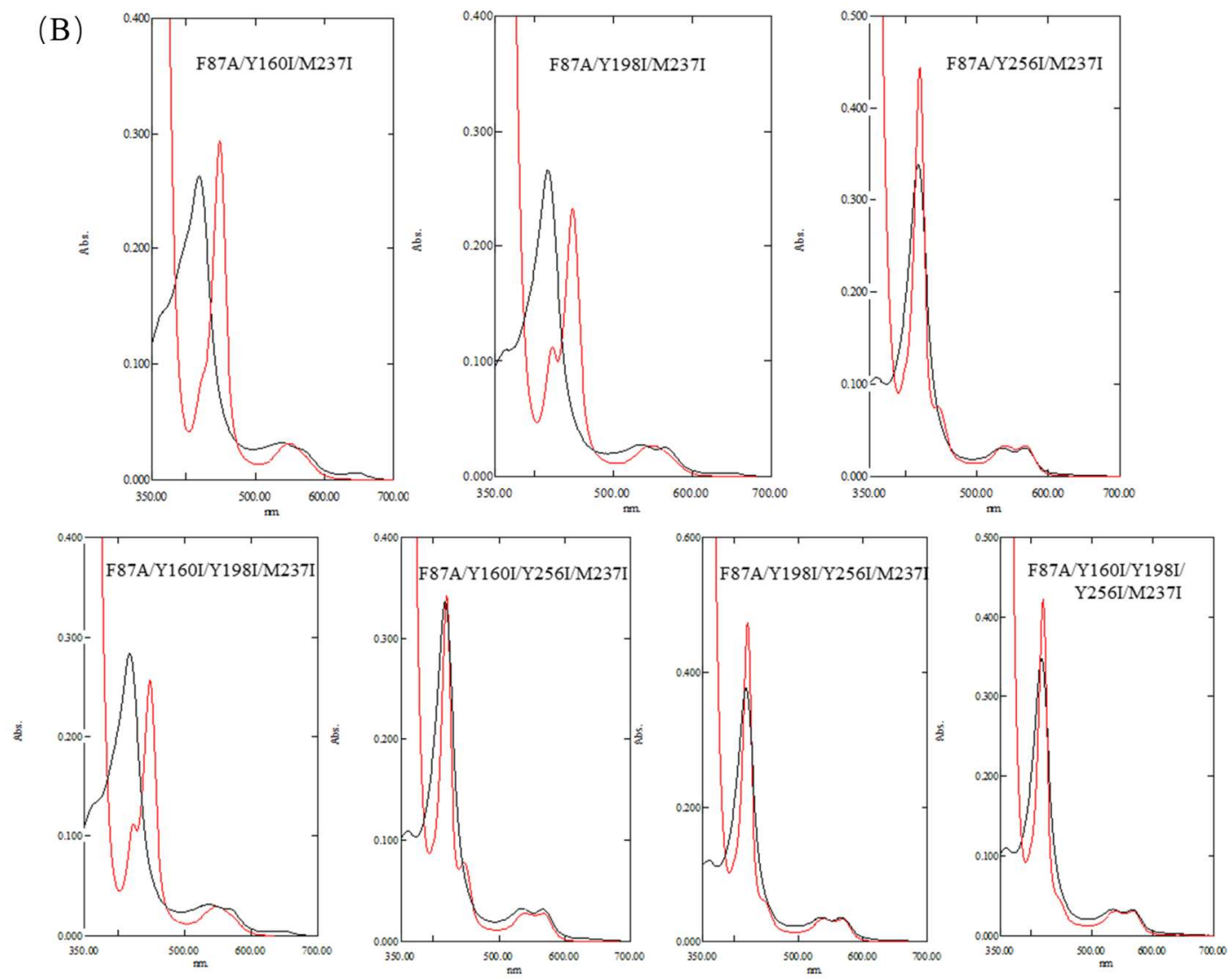

Figure S14 A) SDS page of the combinational mutants of P450BM3. B) UV/visible spectral changes of the combinational mutants of P450BM3 (black line) upon addition of $\mathrm{Na}_{2} \mathrm{~S}_{2} \mathrm{O}_{4}$ (red line) for the formation of a ferrous $\mathrm{CO}$ complex through the reduction of ferric heme. 

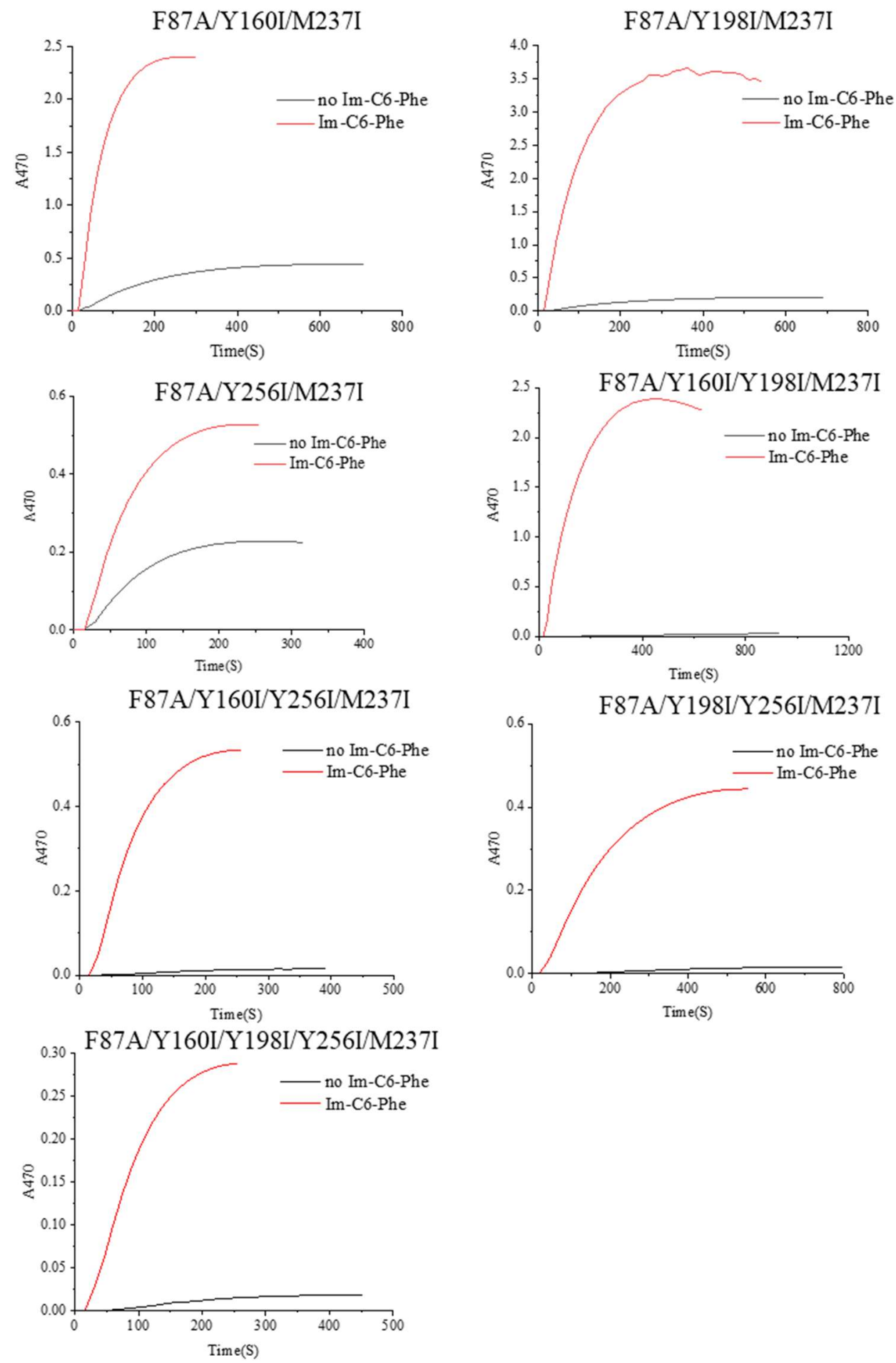

Figure S15 Time course of the absorbance at $470 \mathrm{~nm}$ for the oxidation of guaiacol catalyzed by the combinational mutants of $\mathrm{P} 450 \mathrm{BM} 3$ by addition of $\mathrm{H}_{2} \mathrm{O}_{2}(20 \mathrm{mM})$ in the absence (black line) / presence (redline) of Im-C6-Phe at $25^{\circ} \mathrm{C}$. 

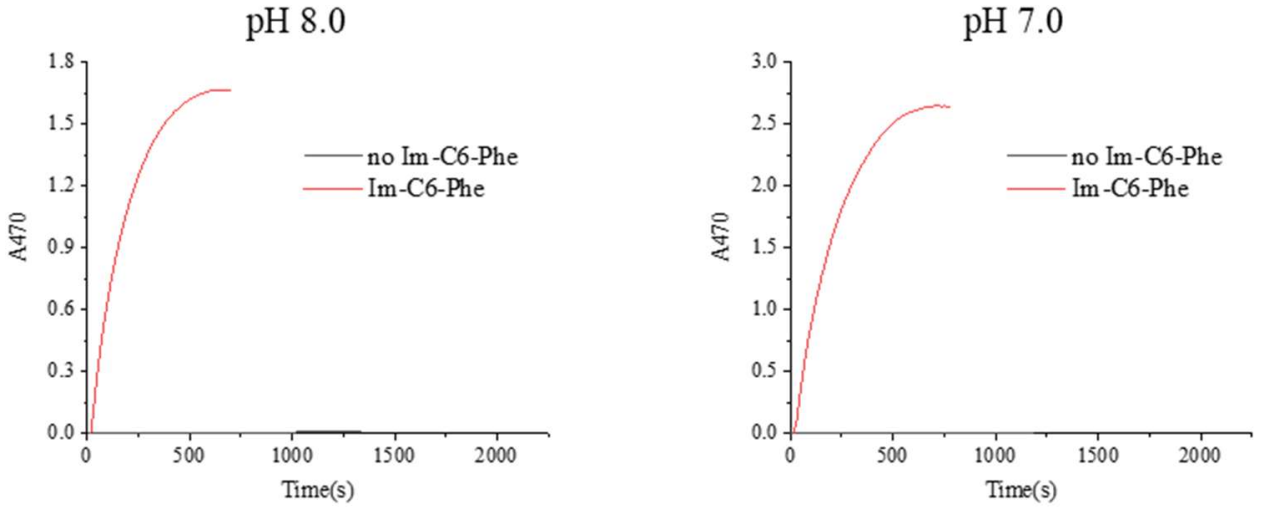

Figure S16 Time course of the absorbance at $470 \mathrm{~nm}$ for the oxidation of guaiacol catalyzed by F87A/Y198I/M237I by addition of $\mathrm{H}_{2} \mathrm{O}_{2}(20 \mathrm{mM})$ in the absence (black line) / presence (red line) of Im-C6-Phe in $0.1 \mathrm{M} \mathrm{pH} \mathrm{8.0} \mathrm{/} 0.05 \mathrm{M} \mathrm{pH} 7.0$ phosphate buffer at $25^{\circ} \mathrm{C}$. ${ }^{*}$ In the case of $0.1 \mathrm{M} \mathrm{pH} 8.0$ phosphate buffer, the TTN of guaiacol oxidation catalyzed by F87A/Y198I/M237I is 5046; In the case of $0.05 \mathrm{M} \mathrm{pH} 7.0$ phosphate buffer, the TTN of guaiacol oxidation catalyzed by F87A/Y198I/M237I is 7912.
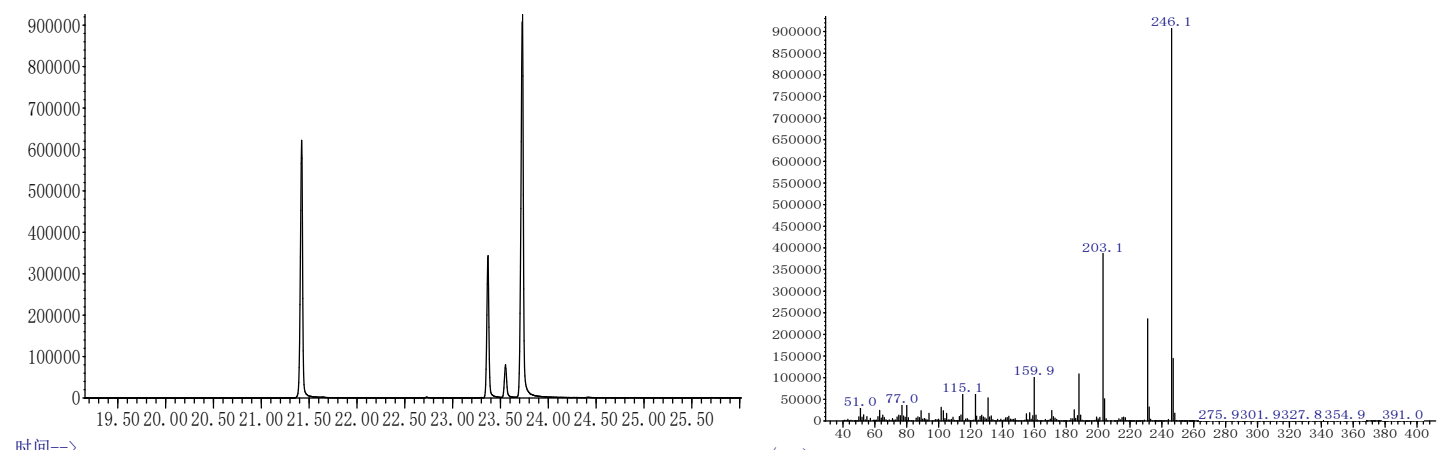

Figure S17 Typical GC-MS analyses for the oxidation of guaiacol catalyzed by P450BM3 mutants by addition of $\mathrm{H}_{2} \mathrm{O}_{2}(20 \mathrm{mM})$ in the presence of Im-C6-Phe at $25^{\circ} \mathrm{C}$. 


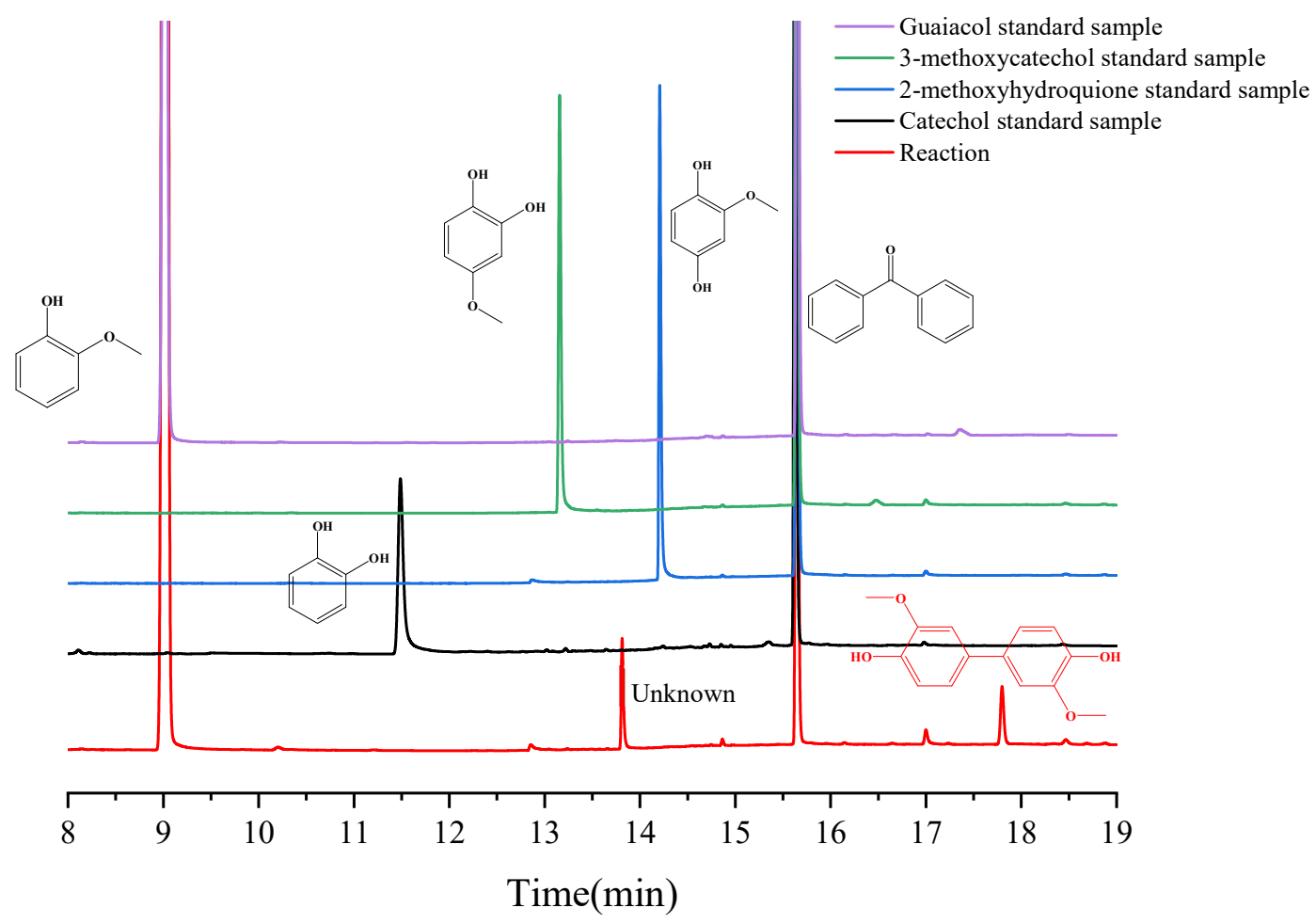

Figure S18 Typical GC analyses for the oxidation of guaiacol catalyzed by P450BM3 mutants by addition of $\mathrm{H}_{2} \mathrm{O}_{2}(20 \mathrm{mM})$ in the presence of Im-C6-Phe at $25^{\circ} \mathrm{C}$. $*$ The peak at 15.7 min dedicates to benzophenone which is added as an internal standard for easily comparing these different samples. 
F87A

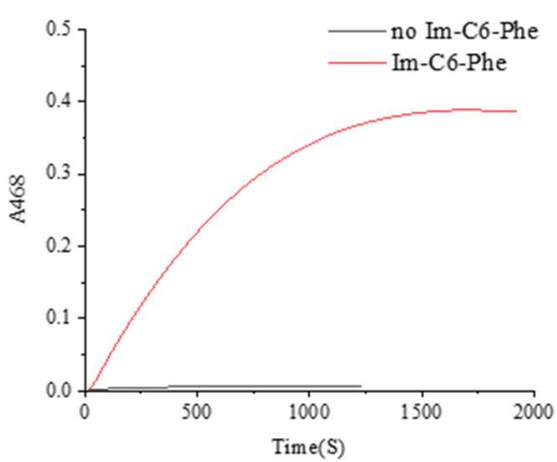

F87A/Y160I/Y256I

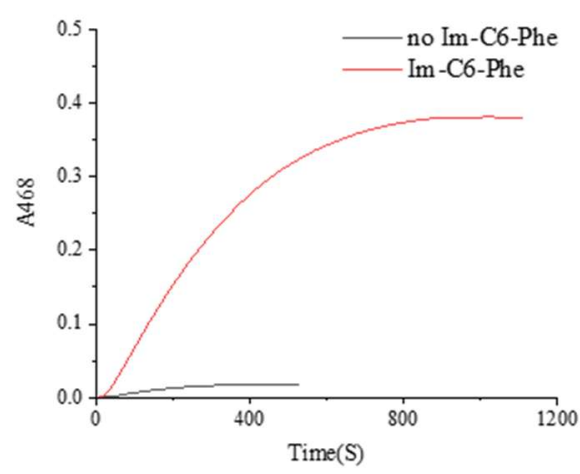

F87A/Y160I/Y198I/Y256I

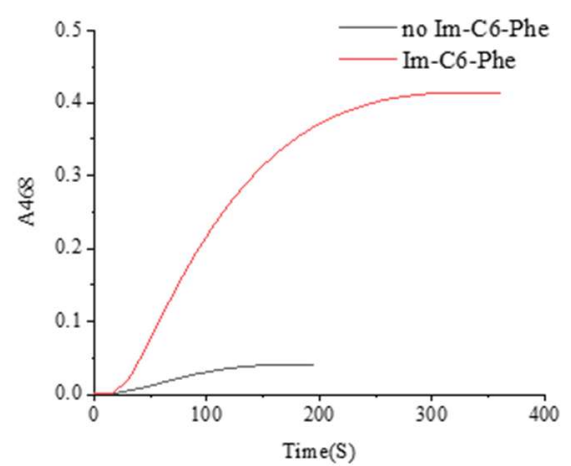

F87A/Y160I/Y198I

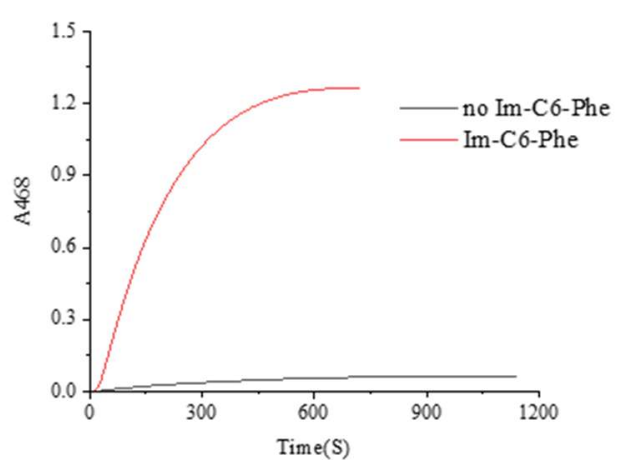

F87A/Y198I/Y256I

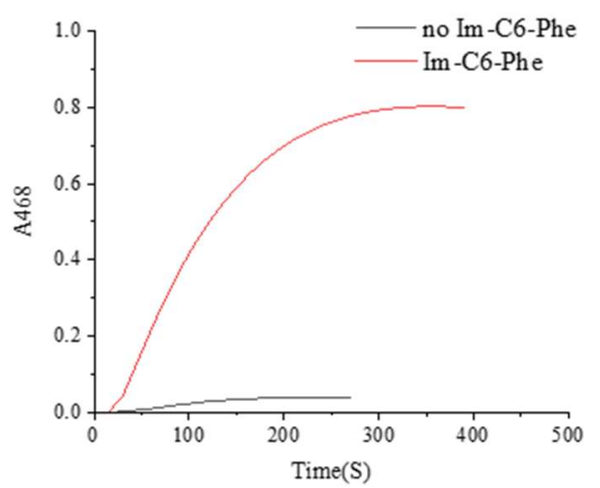

F87A/Y160I/M237I

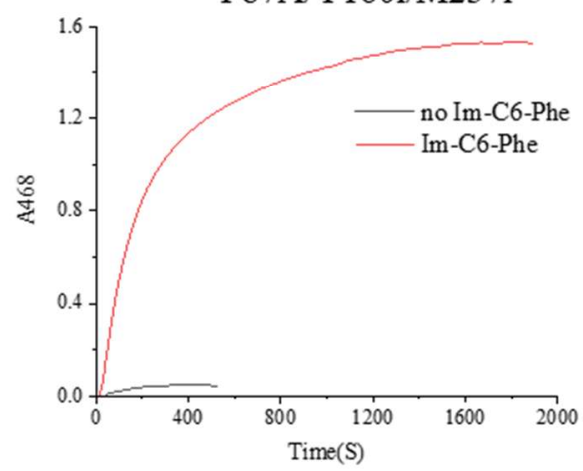




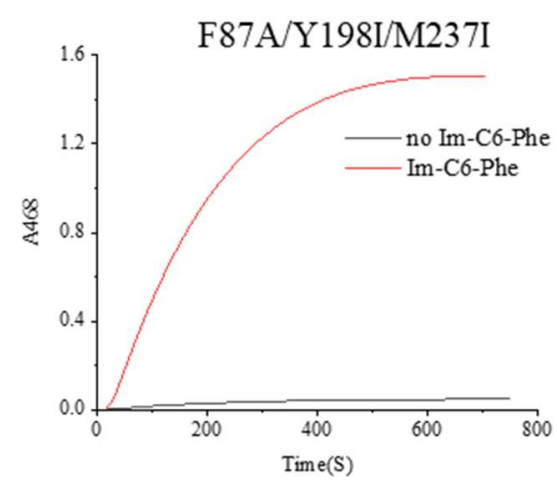

F87A/Y160I/Y198I/M237I

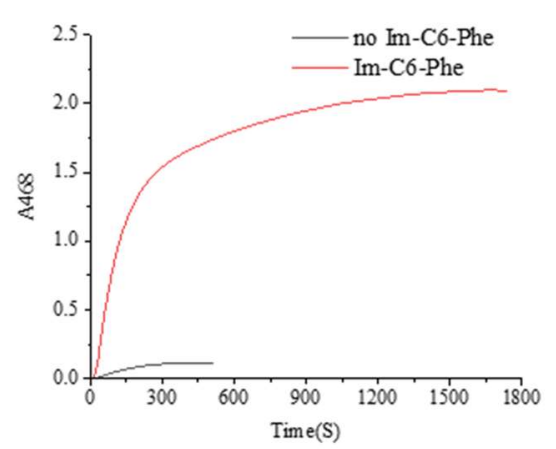

F87A/Y198I/Y256I/M237I

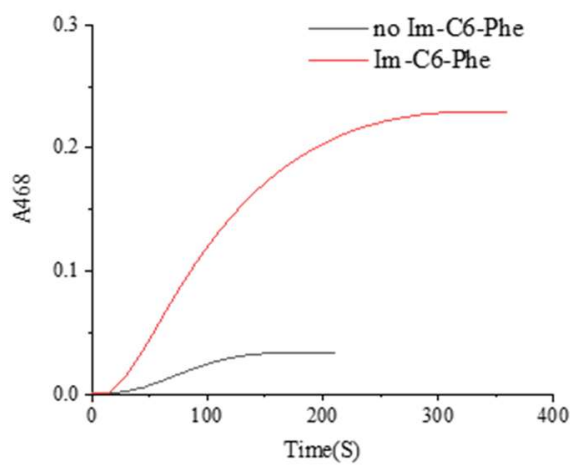

F87A/Y256I/M237I

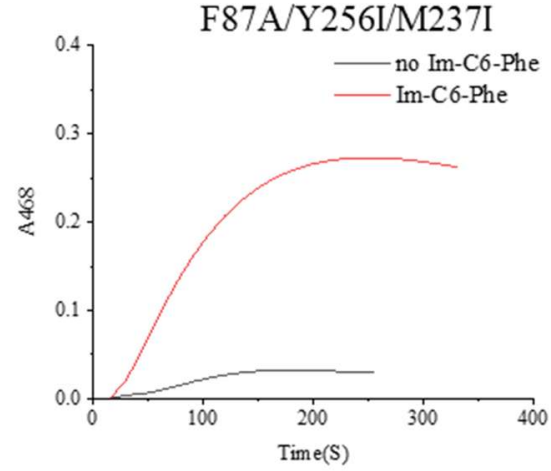

F87A/Y160I/Y256I/M237I



F87A/Y160I/Y198I/Y256I/M237I

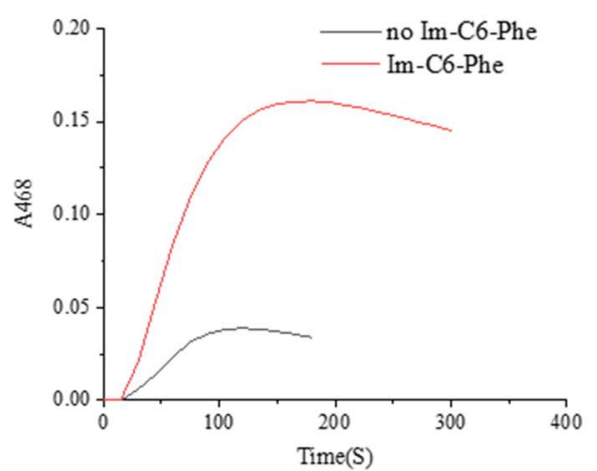

Figure S19 Time course of the absorbance at $468 \mathrm{~nm}$ for the oxidation of 2,6-dimethoxyphenol catalyzed byP450 mutants by addition of $\mathrm{H}_{2} \mathrm{O}_{2}(20 \mathrm{mM})$ in the absence (black line) / presence (red line) of Im-C6-Phe in $0.05 \mathrm{M} \mathrm{pH} 7.0$ phosphate buffer at $25^{\circ} \mathrm{C}$. 



Figure S20 Typical GC-MS analyses for the oxidation of 2,6-dimethoxyphenol catalyzed by P450BM3 mutants by addition of $\mathrm{H}_{2} \mathrm{O}_{2}(20 \mathrm{mM})$ in the presence of Im-C6-Phe at $25{ }^{\circ} \mathrm{C}$.

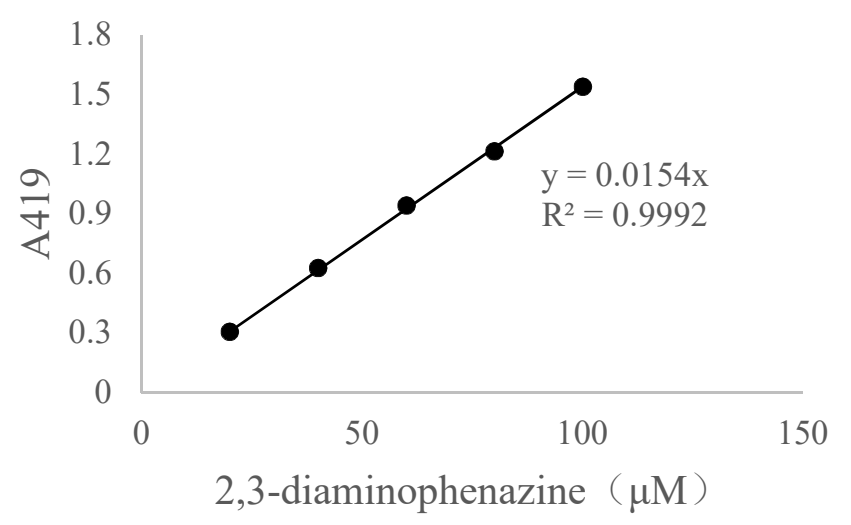

Figure S21 Correlation plot between 2,3-diaminophenazine concentration and absorbance intensity at $419 \mathrm{~nm}$. 
F87A
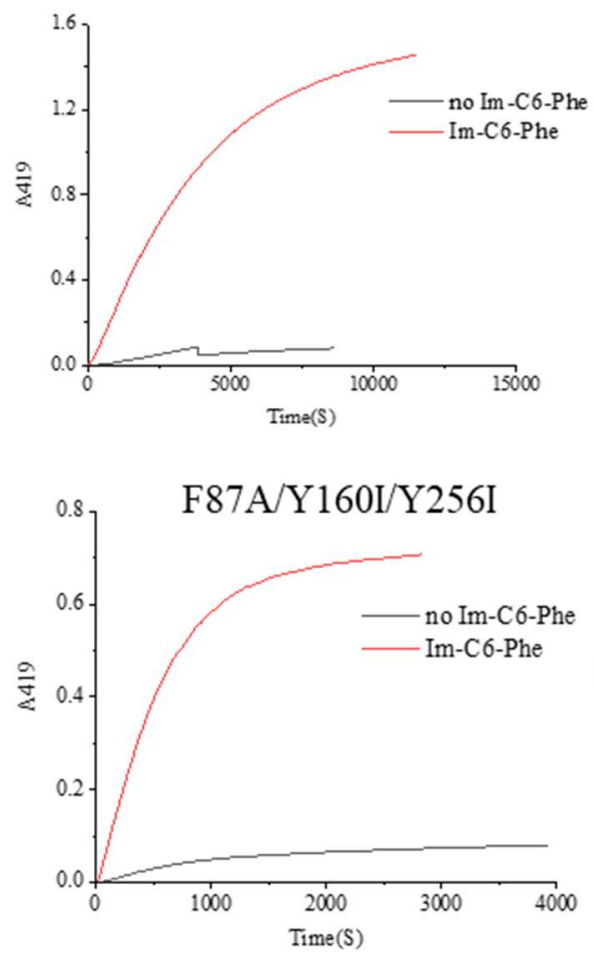

F87A/Y160I/Y198I/Y256I

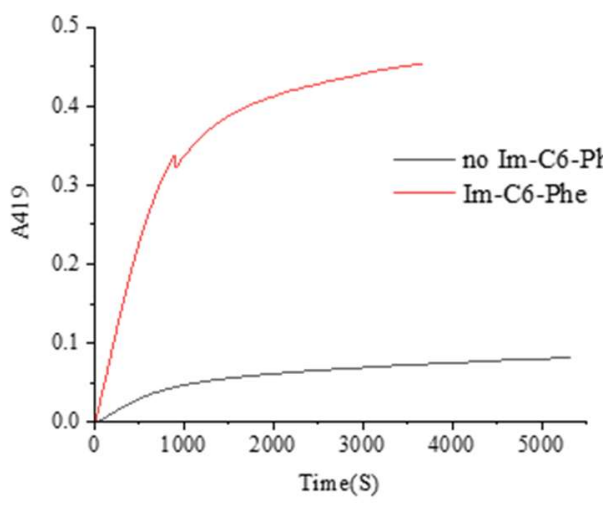

F87A/Y160I/Y198I

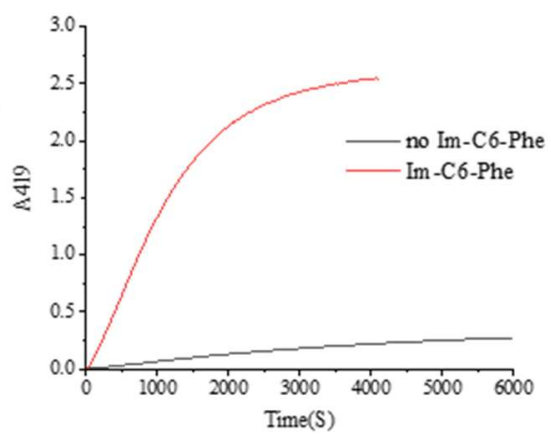

F87A/Y198I/Y256I

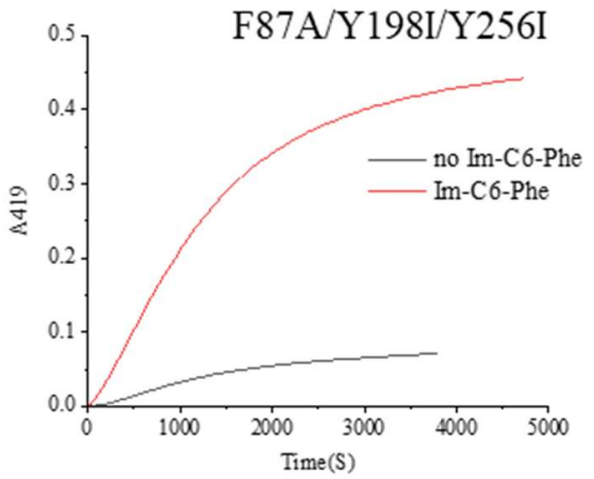

F87A/Y160I/M237I

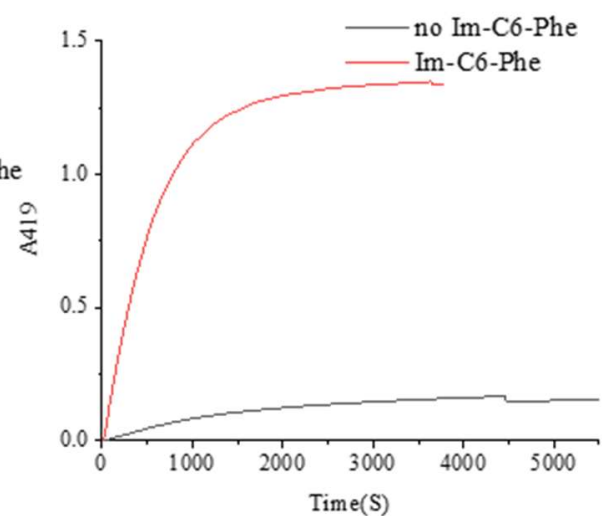


F87A/Y198I/M237I
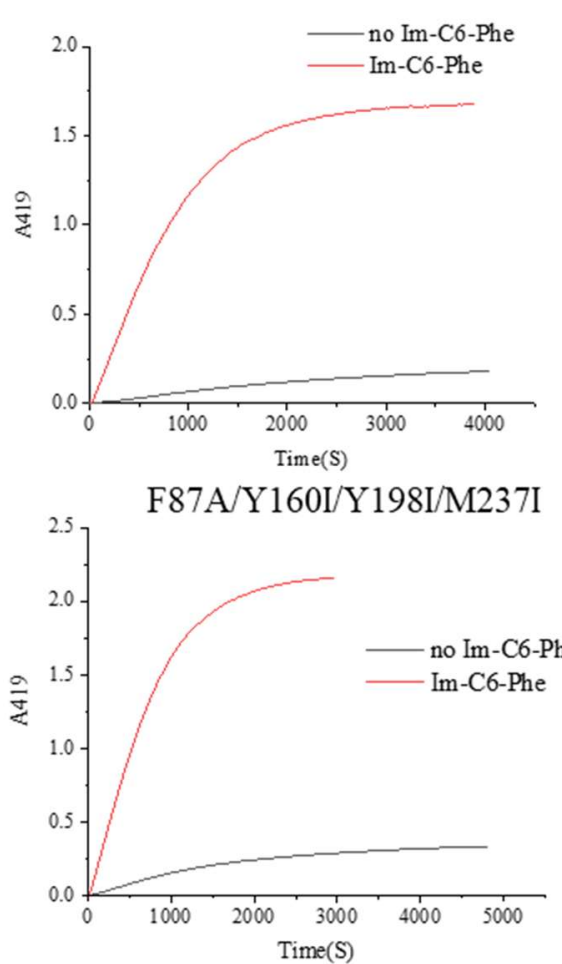

F87A/Y198I/Y256I/M237I

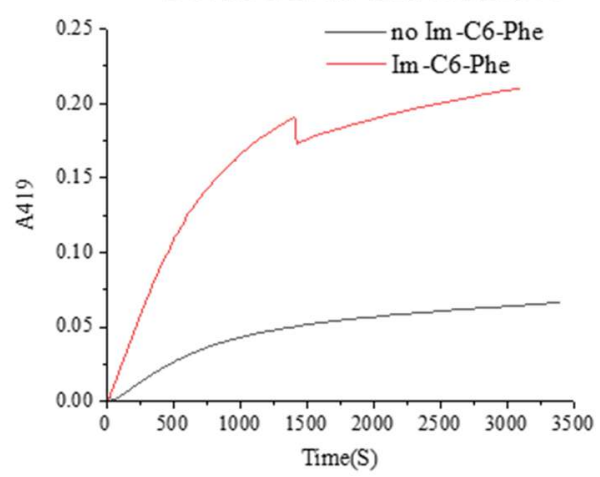

F87A/Y256I/M237I

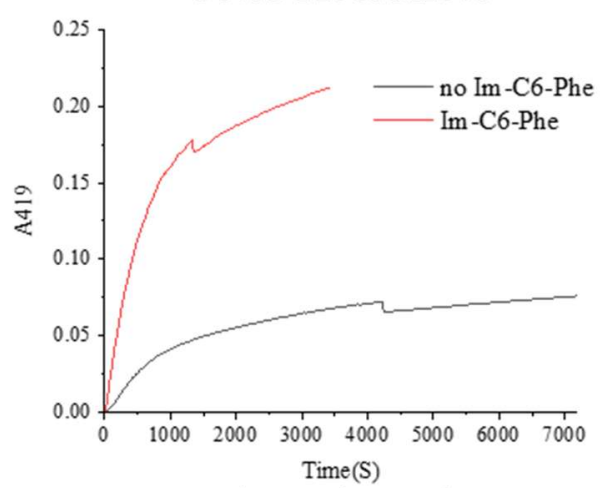

F87A/Y160I/Y256I/M237I

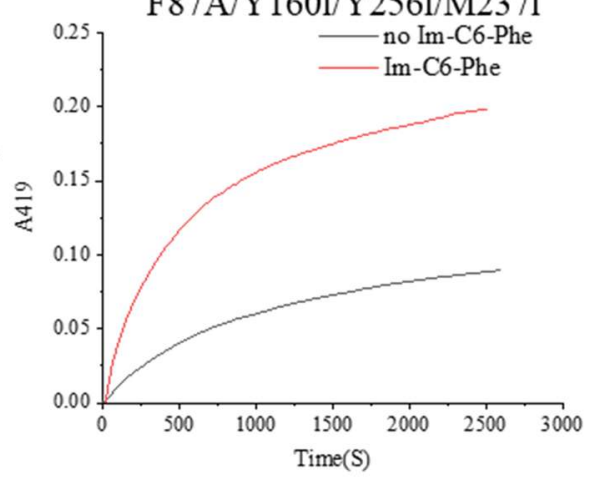

F87A/Y160I/Y198I/Y256I/M237I

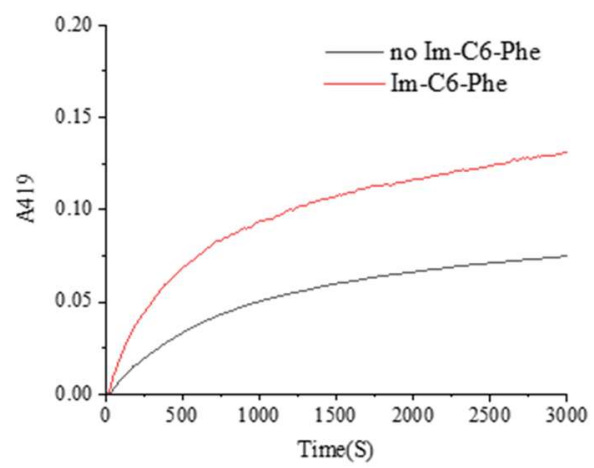

Figure S22 Time course of the absorbance at $419 \mathrm{~nm}$ for the oxidation of $o$-phenylenediamine catalyzed by $\mathrm{P} 450$ mutants by addition of $\mathrm{H}_{2} \mathrm{O}_{2}(20 \mathrm{mM})$ in the absence (black line) / presence (red line) of Im-C6-Phe in $0.05 \mathrm{M} \mathrm{pH} 7.0$ phosphate buffer at $25^{\circ} \mathrm{C}$. 

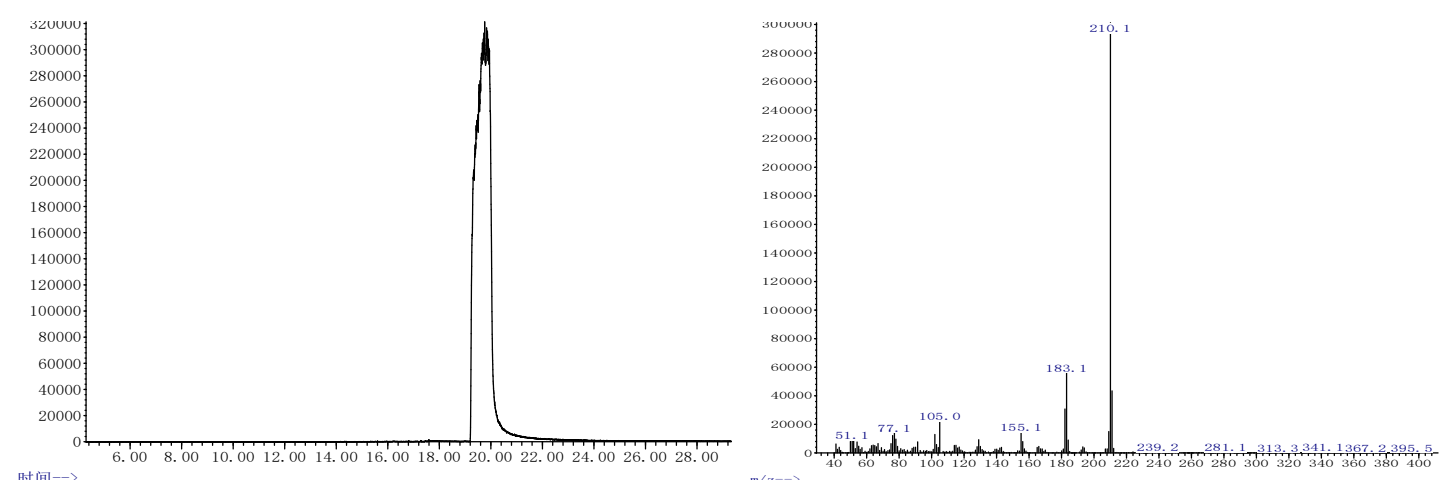

Figure S23 Typical GC-MS analyses for the oxidation of $o$-phenylenediamine catalyzed by P450BM3 mutants by addition of $\mathrm{H}_{2} \mathrm{O}_{2}(20 \mathrm{mM})$ in the presence of Im-C6-Phe at $25{ }^{\circ} \mathrm{C}$. 


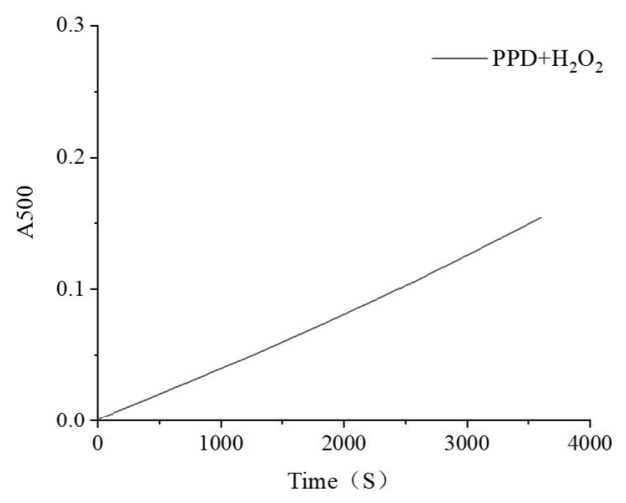

F87A
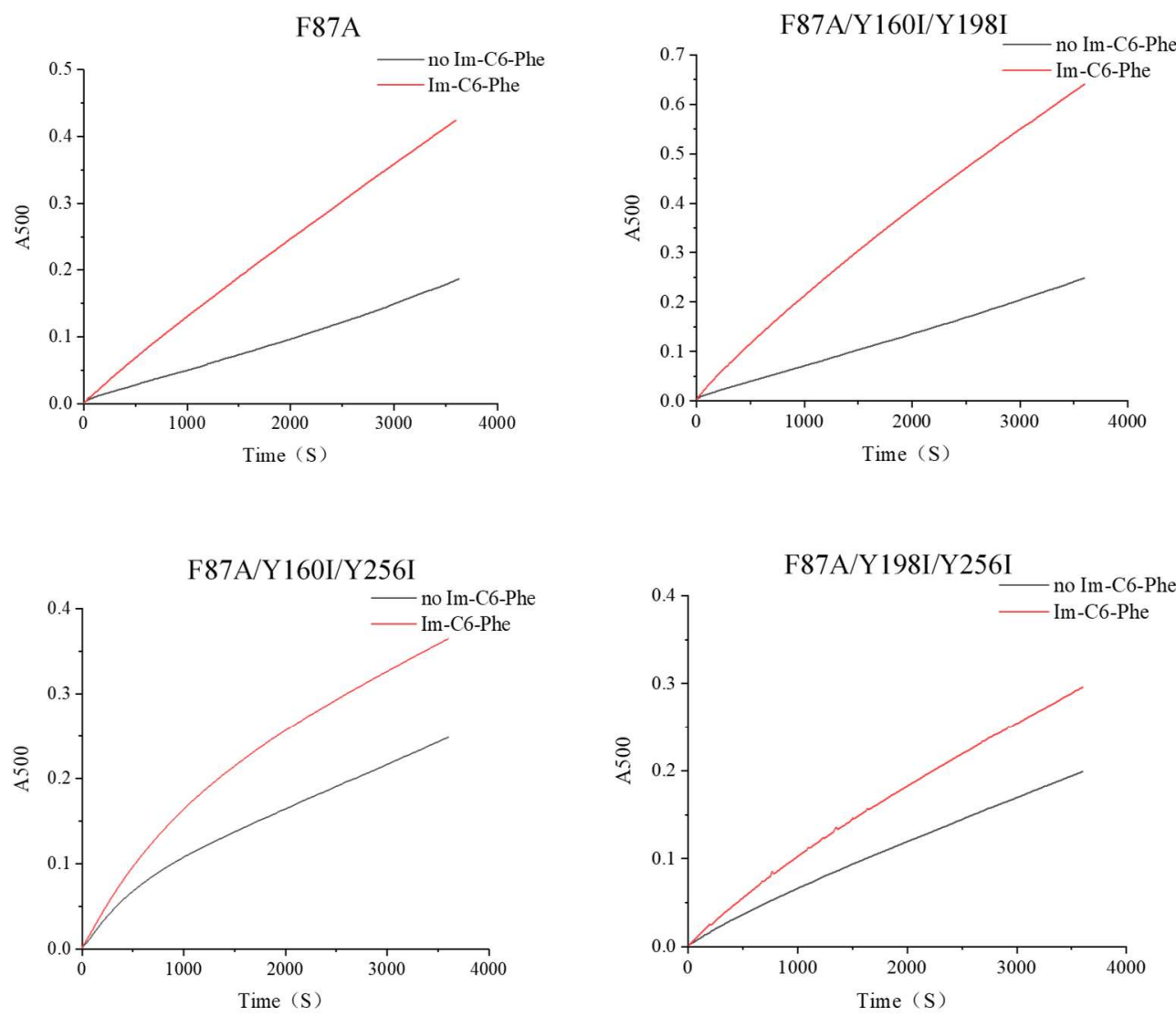

F87A/Y160I/Y198I/Y256I

F87A/Y160I/M237I
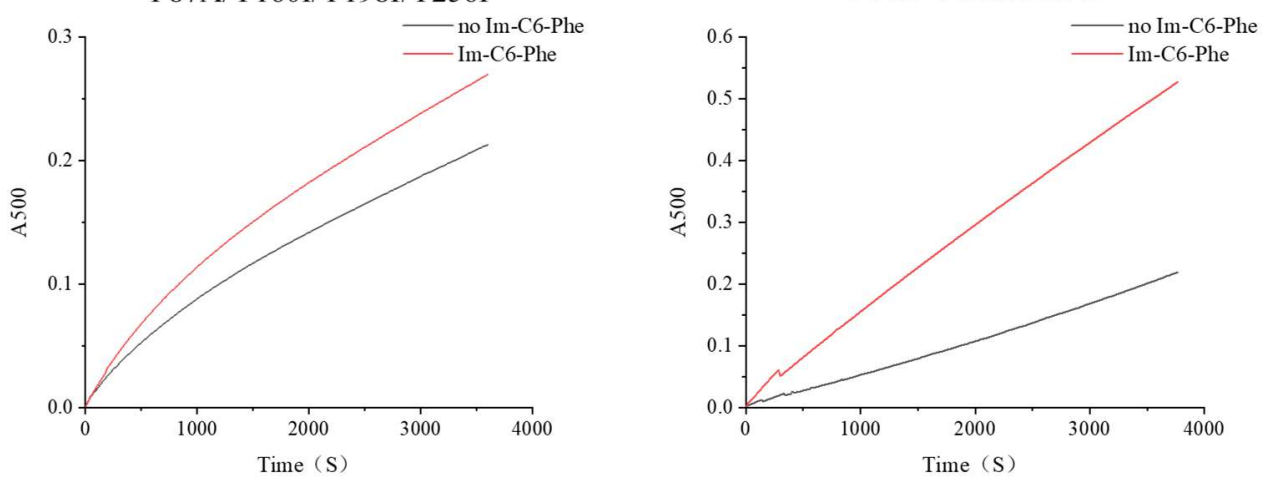

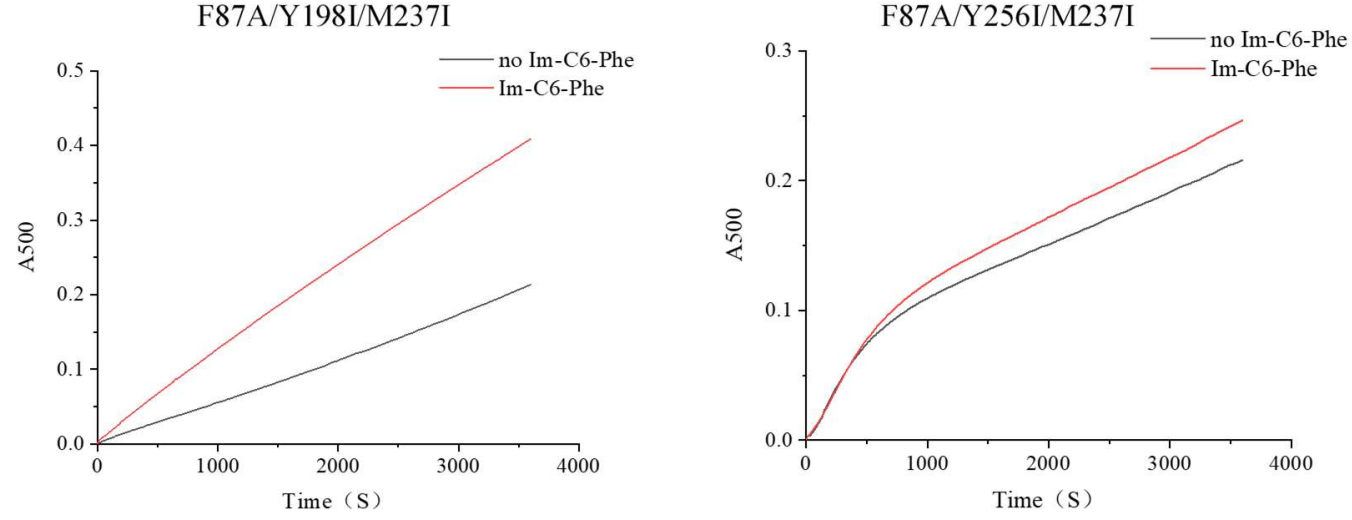

F87A/Y160I/Y198I/M237I
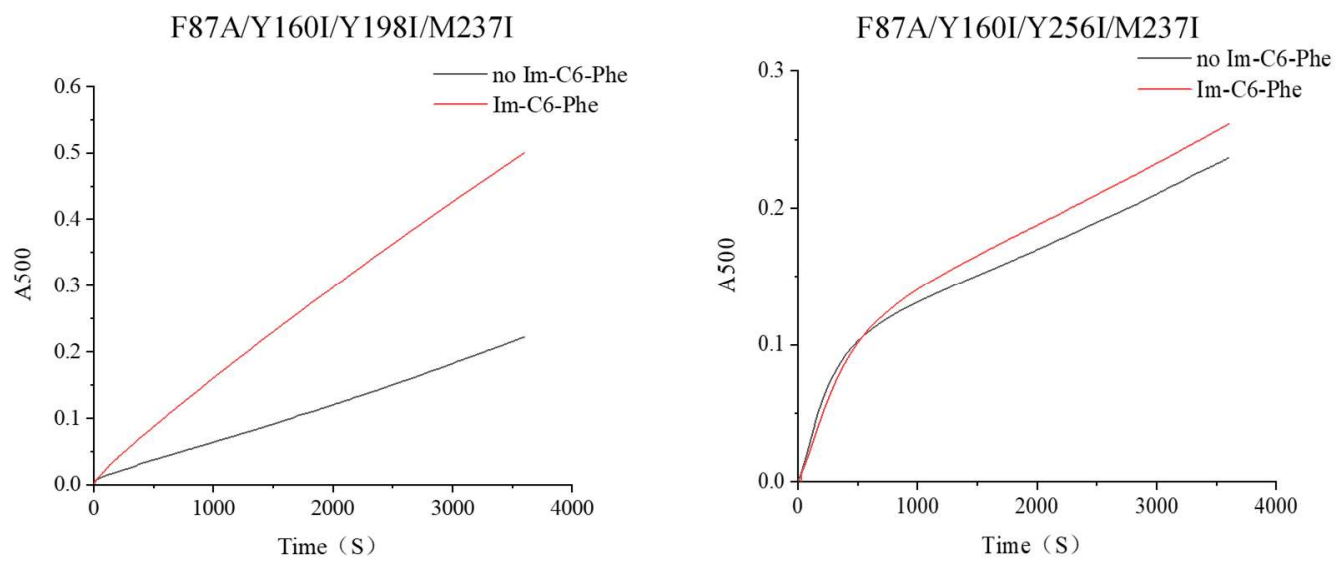

F87A/Y198I/Y256I/M237I
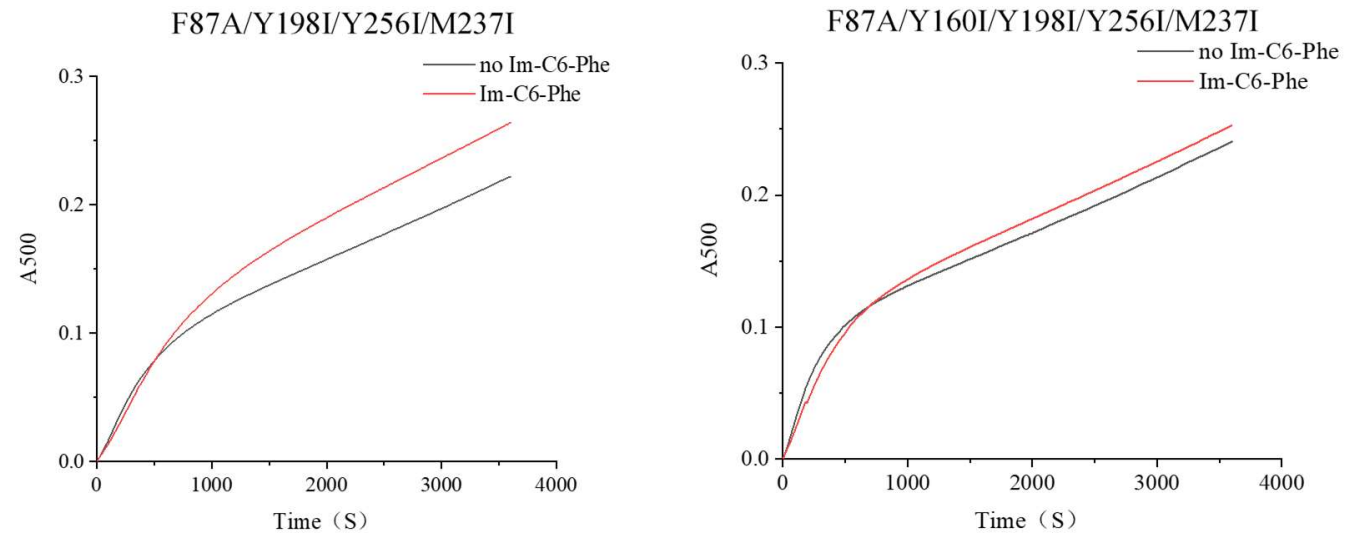

Figure S24 Time course of the absorbance at $500 \mathrm{~nm}$ for the oxidation of $p$-phenylenediamine catalyzed by P450BM3 mutants by addition of $\mathrm{H}_{2} \mathrm{O}_{2}(20 \mathrm{mM})$ in the absence (black line) / presence (red line) of Im-C6-Phe for 1 hour in $\mathrm{pH} 7.0$ phosphate buffer at $25^{\circ} \mathrm{C}$. 

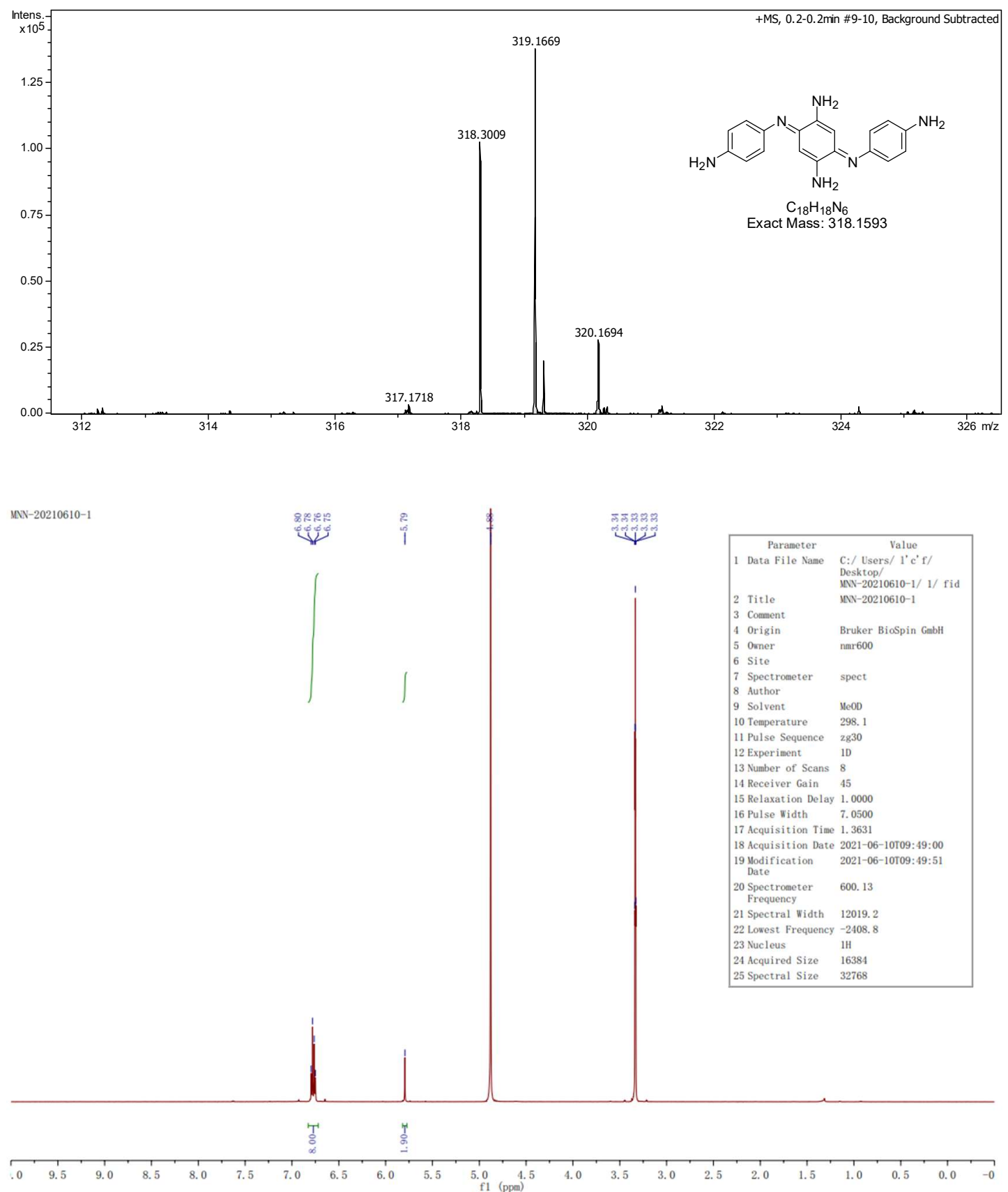

${ }^{1} \mathrm{H}$ NMR (600 MHz, $\left.\mathrm{CD}_{3} \mathrm{OD}\right): \delta 6.75-6.80(\mathrm{~m}, 8 \mathrm{H}), 5.79(\mathrm{~s}, 2 \mathrm{H})$.

Figure S25. The spectra of ESI-MS (top) and ${ }^{1} \mathrm{H}$ NMR (bottom) for the product identification in the oxidation of $p$-phenylenediamine catalyzed by $\mathrm{P} 450 \mathrm{BM} 3$ mutants by addition of $\mathrm{H}_{2} \mathrm{O}_{2}(20 \mathrm{mM})$ in the presence of Im-C6-Phe at $25^{\circ} \mathrm{C}$. 

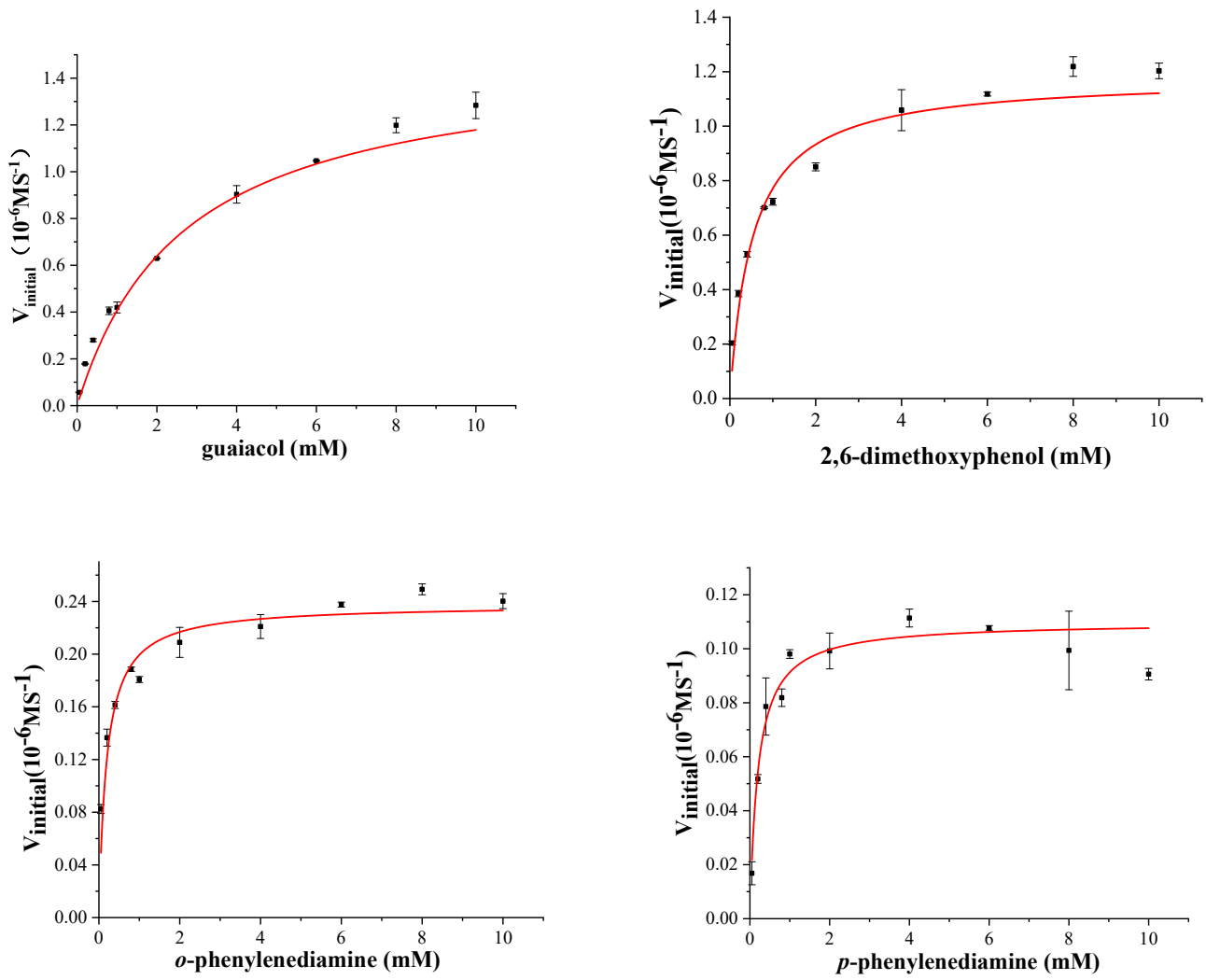

Figure S26 Steady state kinetic plot for guaiacol, 2,6-dimethoxyphenol, o-phenylenediamine, $p$ phenylenediamine. Each data point represents the mean and standard deviation from two measurements. 

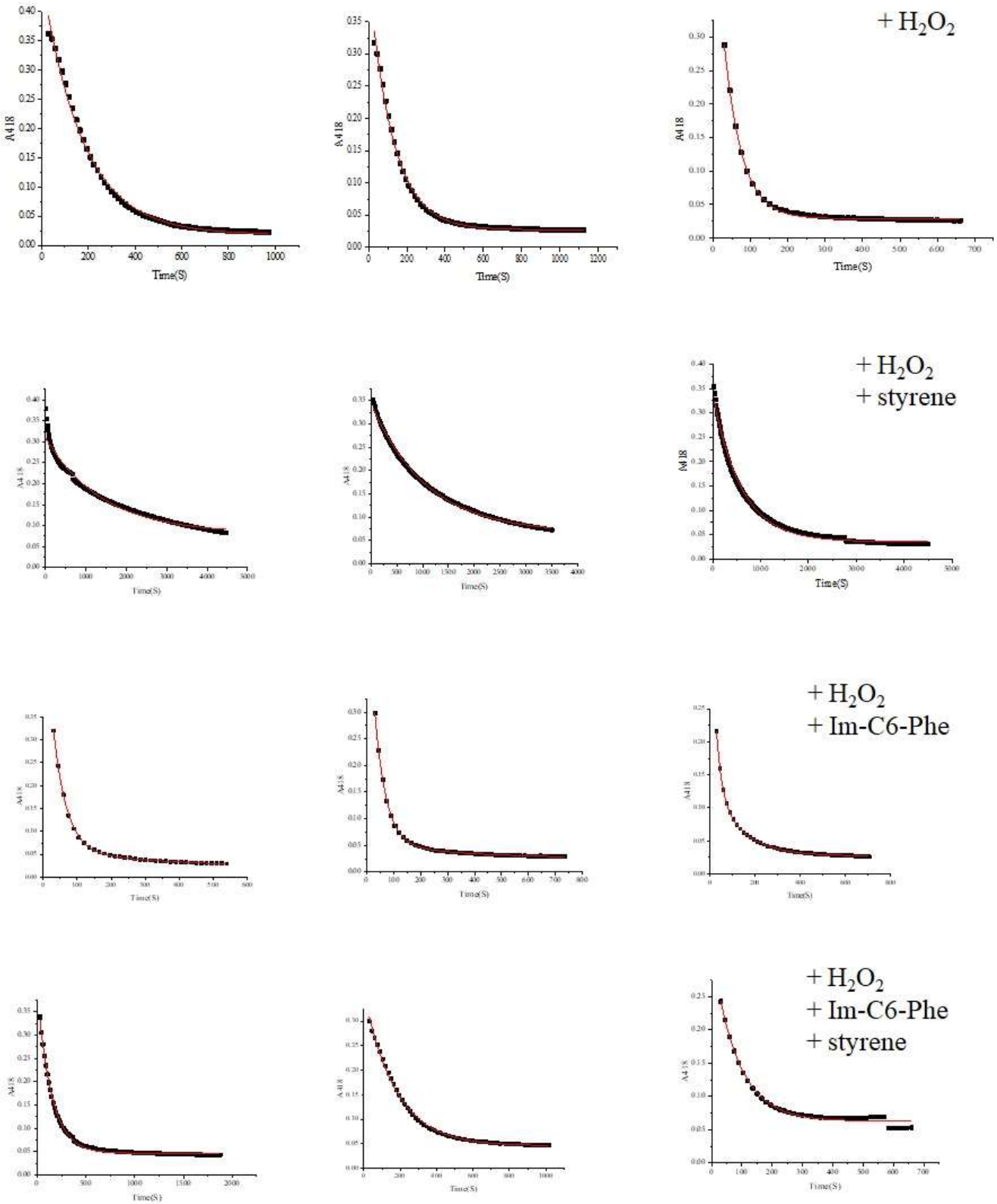

Figure S27 Time-course of absorbance at $418 \mathrm{~nm}$ for the heme degradation of P450BM3 mutants $(2 \mu \mathrm{M})$ with $\mathrm{H}_{2} \mathrm{O}_{2}$ at $25{ }^{\circ} \mathrm{C}$. ${ }^{*}$ First line: F87A/Y160I/Y198I; second line: F87A/Y198I/M237I; third line: F87A/Y160I/Y198I/M237I. ** First row: $\mathrm{H}_{2} \mathrm{O}_{2}(20 \mathrm{mM})$ was added; second row: $\mathrm{H}_{2} \mathrm{O}_{2}$ $(20 \mathrm{mM})$ and styrene $(4 \mathrm{mM})$ was added; third row: Im-C6-Phe $(4 \mathrm{mM})$ and $\mathrm{H}_{2} \mathrm{O}_{2}(20 \mathrm{mM})$ was added; fourth row: Im-C6-Phe $(4 \mathrm{mM}), \mathrm{H}_{2} \mathrm{O}_{2}(20 \mathrm{mM})$, and styrene $(4 \mathrm{mM})$ was added. ${ }^{* * *}$ The solid red line shows least square curve-fit with a single exponential function of Abs $=\mathrm{A} 0+\mathrm{A} \exp (-$ $\left.k_{o b s} \mathrm{t}\right)\left(1^{\text {st }}, 2^{\text {nd }}\right.$, and $4^{\text {th }}$ row $)$ and a double exponential function of Abs $=\mathrm{A} 0+\mathrm{A} 1 \exp \left(-k_{o b s} \mathrm{t}\right)+$ $\mathrm{A} 2 \exp \left(-k_{o b s 2} \mathrm{t}\right)\left(3^{\text {rd }}\right.$ row). ${ }^{* * * *}$ The observed heme decay rates are summarized in Table S11. 
(a)

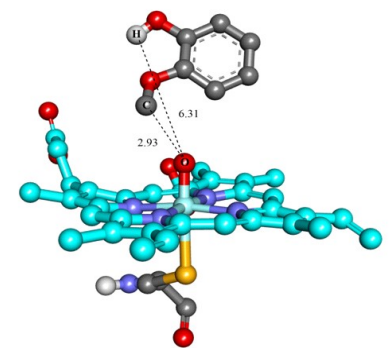

3321/4000

(b)

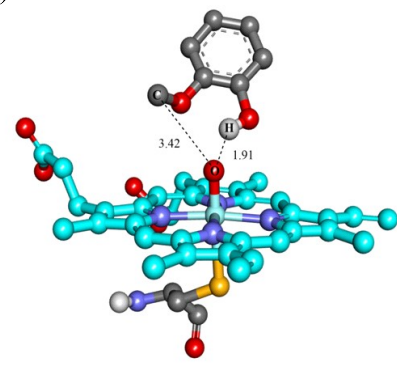

$3852 / 4000$

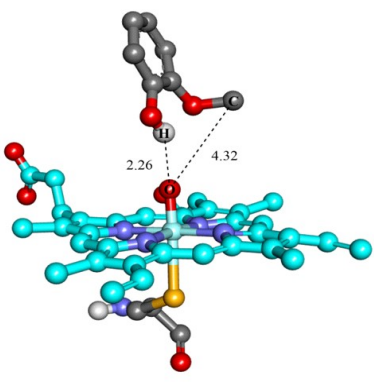

629/4000

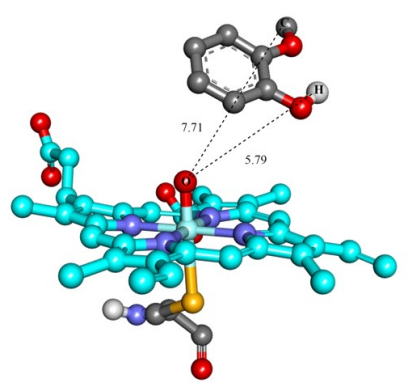

$142 / 4000$

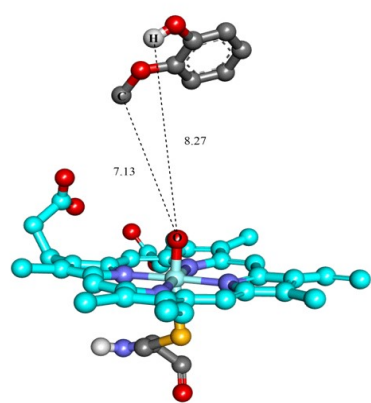

50/4000

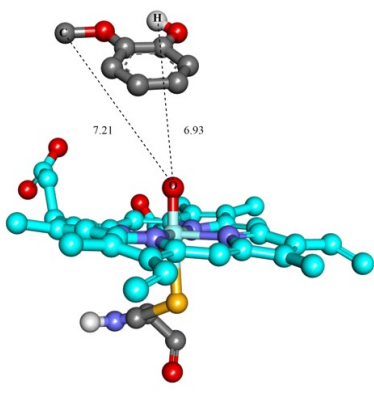

6/4000

Figure S28. Three most populated clusters obtained from the clustering analysis of the MD trajectory. (a) F87A mutant. (b) F87A/Y160I/Y198I/M237I mutant in the absence of DFSM. 
(a)

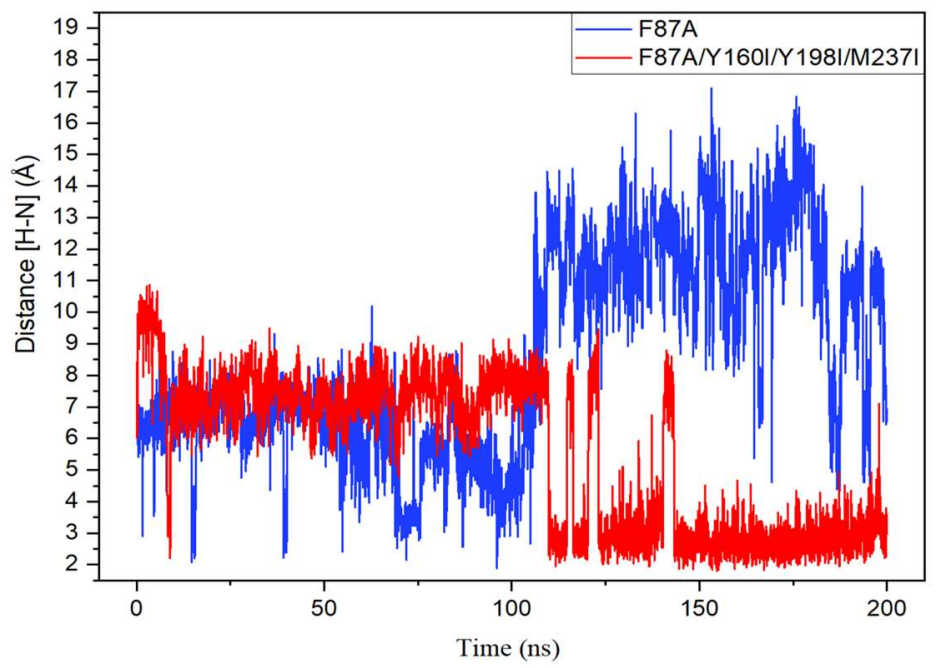

(b)

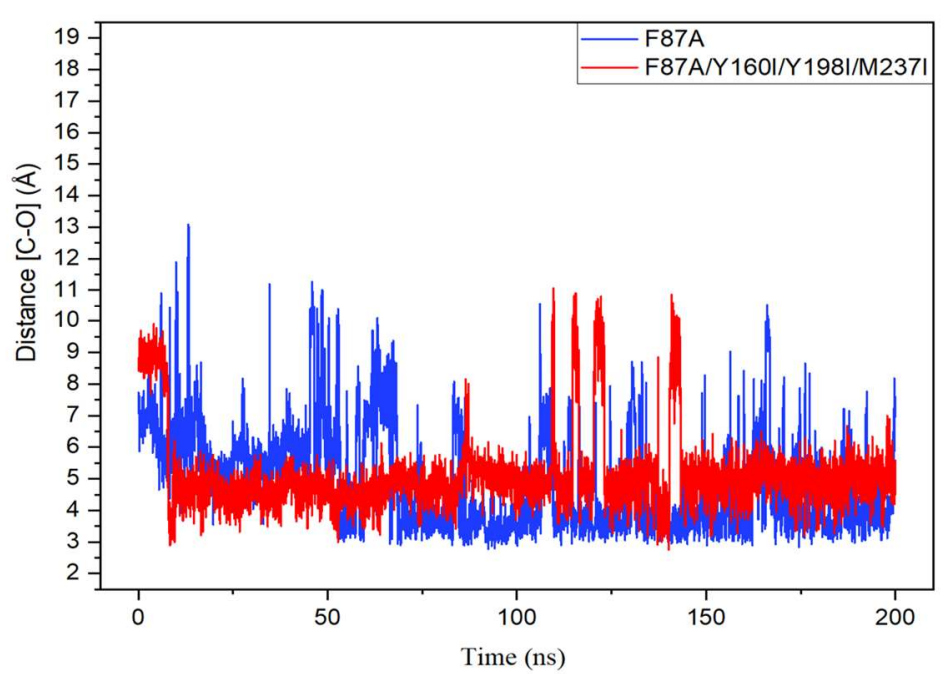

(c)

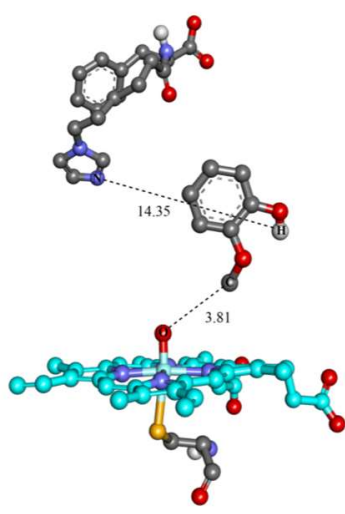

F87A

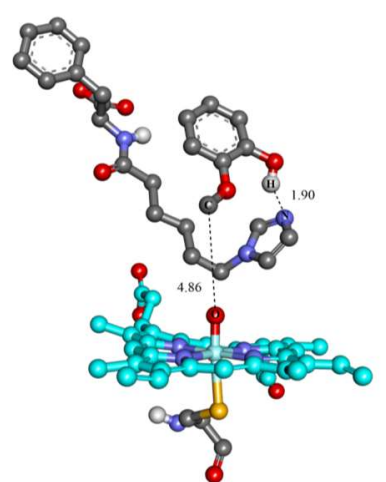

F87A/Y160I/Y198I/M237I

Figure S29. (a) The distance fluctuation of N(DFSM)-H(substrate) in F87A and F87A/Y160I/Y198I/M237I in the presence of DFSM; (b) The distance fluctuation of $\mathrm{C}$ (substrate)-O(Cpd I) in F87A and F87A/Y160I/Y198I/M237I mutant in the presence of DFSM; (c) The representative structures from the MD trajectories of F87A and F87A/Y160I/Y198I/M237I in the presence of DFSM. 

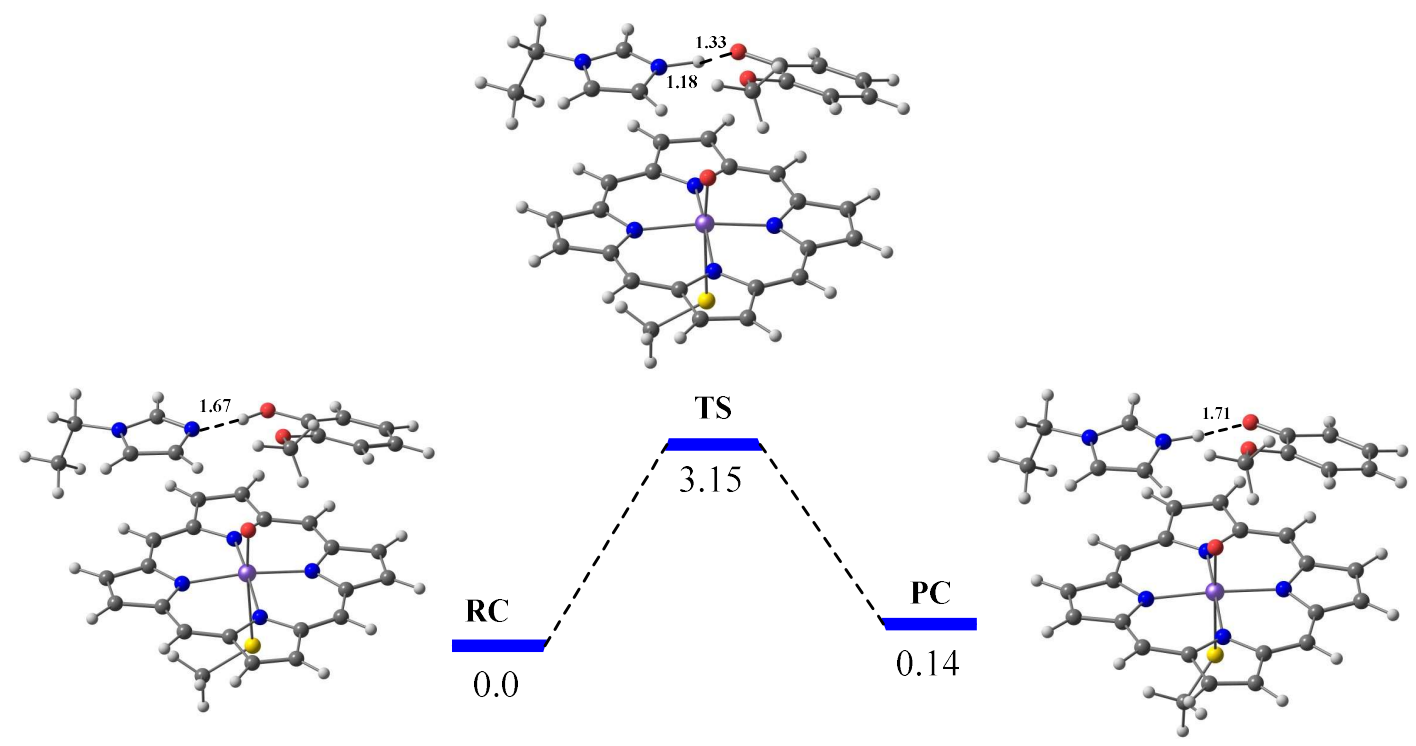

Figure S30. QM(B3LYP/B2)-calculated Gibbs energy barrier (in kcal/mol) for DFSM-mediated PCET process to generate the substrate radical.

Table S1 Screening the mutations F87A/Y160X of P450BM3 heme domain for the oxidation of guaiacol with $\mathrm{H}_{2} \mathrm{O}_{2}$ in the absence/presence of Im-C6-Phe ${ }^{[a]}$

\begin{tabular}{ccc}
\hline Mutation of Y160X & Im-C6-Phe & TTN $^{[\mathrm{b}]}$ \\
\hline F87A & - & 10 \\
F87A & + & $49 \pm 4$ \\
Y160A & - & 12 \\
Y160A & + & 45 \\
Y160I & - & 45 \\
Y160I & + & $149 \pm 6$ \\
Y160L & - & 52 \\
Y160L & + & 108 \\
Y160V & - & 19 \\
Y160V & + & 107 \\
Y160G & - & 5 \\
Y160G & + & 49 \\
\hline
\end{tabular}

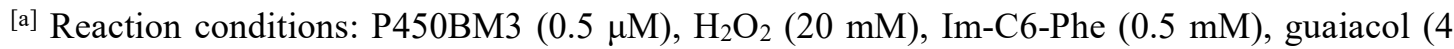
$\mathrm{mM})$ in $\mathrm{pH} 8.0$ phosphate buffer at $25^{\circ} \mathrm{C}$. ${ }^{[b]} \mathrm{TTN}$ : Total turnover numbers were estimated by the highest absorption peak at $470 \mathrm{~nm}$. 
Table S2 Screening tyrosine of P450BM3-F87A heme domain for the oxidation of guaiacol with $\mathrm{H}_{2} \mathrm{O}_{2}$ in the absence/presence of Im-C6-Phe ${ }^{[\mathrm{a}]}$

\begin{tabular}{ccc}
\hline Mutation of tyrosine & Im-C6-Phe & TTN $^{[\mathrm{b}]}$ \\
\hline Y51I & - & 6 \\
Y51I & + & 9 \\
Y115I & - & 12 \\
Y115I & + & 78 \\
Y166I & - & 9 \\
Y166I & + & 49 \\
Y198I & - & 15 \\
Y198I & + & $249 \pm 7$ \\
Y256I & - & 26 \\
Y256I & + & $317 \pm 7$ \\
Y278I & - & 12 \\
Y278I & + & 60 \\
Y305I & - & 13 \\
Y305I & + & 39 \\
Y313I & - & 6 \\
Y313I & + & 45 \\
Y334I & - & 9 \\
Y334I & + & 45 \\
Y345I & - & 16 \\
Y345I & + & 60 \\
Y429I & - & 9 \\
Y429I & + & 55 \\
\hline
\end{tabular}

${ }^{[a]}$ Reaction conditions: P450BM3 $(0.5 \mu \mathrm{M}), \mathrm{H}_{2} \mathrm{O}_{2}(20 \mathrm{mM})$, Im-C6-Phe $(0.5 \mathrm{mM})$, guaiacol (4 $\mathrm{mM})$ in $\mathrm{pH} 8.0$ phosphate buffer at $25^{\circ} \mathrm{C}$. ${ }^{[b]} \mathrm{TTN}$ : Total turnover numbers were estimated by the highest absorption peak at $470 \mathrm{~nm}$.

Table S3 Oxidation of guaiacol catalyzed by the combinational tyrosine mutants of P450BM3 heme domain with $20 \mathrm{mM} \mathrm{H}_{2} \mathrm{O}_{2}$ in absence/presence of Im-C6-Phe ${ }^{[\mathrm{a}]}$

\begin{tabular}{ccc}
\hline Mutants & Im-C6-Phe & TTN $^{[\mathrm{b}]}$ \\
\hline F87A/Y160I/Y198I & - & 70 \\
F87A/Y160I/Y198I & + & $750 \pm 46$ \\
F87A/Y160I/Y256I & - & 58 \\
F87A/Y160I/Y256I & + & $696 \pm 20$ \\
F87A/Y198I/Y256I & - & 36 \\
F87A/Y198I/Y256I & + & $390 \pm 15$ \\
F87A/Y160I/Y198I/Y256I & - & 68 \\
F87A/Y160I/Y198I/Y256I & + & $599 \pm 31$ \\
\hline
\end{tabular}

[a] Reaction conditions: P450BM3 $(0.5 \mu \mathrm{M}), \mathrm{H}_{2} \mathrm{O}_{2}(20 \mathrm{mM})$, Im-C6-Phe $(0.5 \mathrm{mM})$, guaiacol (4 $\mathrm{mM})$ in $\mathrm{pH} 8.0$ phosphate buffer at $25^{\circ} \mathrm{C}$. ${ }^{[b]} \mathrm{TTN}$ : Total turnover numbers were estimated by the highest absorption peak at $470 \mathrm{~nm}$. 
Table S4 Screening the mutations F87A/F405X of P450BM3 heme domain for the oxidation of guaiacol with $\mathrm{H}_{2} \mathrm{O}_{2}$ in the presence of Im-C6-Phe ${ }^{[a]}$

\begin{tabular}{ccc}
\hline Mutation of F405 & Im-C6-Phe & TTN $^{[\mathrm{b}]}$ \\
\hline F405A & - & 8 \\
F405A & + & 27 \\
F405I & - & 15 \\
F405I & + & 33 \\
F405L & - & 8 \\
F405L & + & 12 \\
F405V & - & 9 \\
F405V & + & 22 \\
\hline
\end{tabular}

${ }^{[a]}$ Reaction conditions: P450BM3 $(0.5 \mu \mathrm{M}), \mathrm{H}_{2} \mathrm{O}_{2}(20 \mathrm{mM})$, Im-C6-Phe $(0.5 \mathrm{mM})$, guaiacol (4 $\mathrm{mM})$ in $\mathrm{pH} 8.0$ phosphate buffer at $25^{\circ} \mathrm{C}$. ${ }^{[b]} \mathrm{TTN}$ : Total turnover numbers were estimated by the highest absorption peak at $470 \mathrm{~nm}$.

Table S5 Screening the mutations F87A/W96X of P450BM3 heme domain for the oxidation of guaiacol with $\mathrm{H}_{2} \mathrm{O}_{2}$ in the presence of Im-C6-Phe ${ }^{[a]}$

\begin{tabular}{ccc}
\hline Mutation of W96 & Im-C6-Phe & TTN $^{[\mathrm{b}]}$ \\
\hline W96A & - & 25 \\
W96A & + & 48 \\
W96I & - & 46 \\
W96I & + & 50 \\
W96L & - & 52 \\
W96L & + & 63 \\
W96V & - & 46 \\
W96V & + & 63 \\
\hline
\end{tabular}

[a] Reaction conditions: P450BM3 $(0.5 \mu \mathrm{M}), \mathrm{H}_{2} \mathrm{O}_{2}(20 \mathrm{mM})$, Im-C6-Phe $(0.5 \mathrm{mM})$, guaiacol (4 $\mathrm{mM})$ in $\mathrm{pH} 8.0$ phosphate buffer at $25^{\circ} \mathrm{C}$. ${ }^{[b]}$ TTN: Total turnover numbers were estimated by the highest absorption peak at $470 \mathrm{~nm}$. 
Table S6 Screening the mutations F87A/M237X of P450BM3 heme domain for the oxidation of guaiacol with $\mathrm{H}_{2} \mathrm{O}_{2}$ in the absence/presence of Im-C6-Phe ${ }^{[a]}$

\begin{tabular}{ccc}
\hline Mutation of M237 & Im-C6-Phe & TTN $^{[\mathrm{b}]}$ \\
\hline M237A & - & 13 \\
M237A & + & 52 \\
M237I & - & 38 \\
M237I & + & $465 \pm 11$ \\
M237L & - & 34 \\
M237L & + & 336 \\
M237V & - & 19 \\
M237V & + & 215 \\
\hline
\end{tabular}

${ }^{\text {a] }}$ Reaction conditions: P450BM3 $(0.5 \mu \mathrm{M}), \mathrm{H}_{2} \mathrm{O}_{2}(20 \mathrm{mM})$, Im-C6-Phe $(0.5 \mathrm{mM})$, guaiacol (4 $\mathrm{mM})$ in $\mathrm{pH} 8.0$ phosphate buffer at $25^{\circ} \mathrm{C}$. ${ }^{[b]} \mathrm{TTN}$ : Total turnover numbers were estimated by the highest absorption peak at $470 \mathrm{~nm}$.

Table S7 Oxidation of guaiacol catalyzed by the combinational mutants of P450BM3 heme domain with $20 \mathrm{mM} \mathrm{H}_{2} \mathrm{O}_{2}$ in the absence/presence of Im-C6-Phe ${ }^{[\mathrm{a}]}$

\begin{tabular}{|c|c|c|}
\hline Mutants / nM & Im-C6-Phe / mM & $\mathrm{TTN}^{[\mathrm{d}]}$ \\
\hline $\mathrm{F} 87 \mathrm{~A} / \mathrm{Y} 160 \mathrm{I} / \mathrm{M} 237 \mathrm{I}^{[\mathrm{b}, \mathrm{c}]} / 500$ & 0 & 133 \\
\hline $\mathrm{F} 87 \mathrm{~A} / \mathrm{Y} 160 \mathrm{I} / \mathrm{M} 237 \mathrm{I}^{[\mathrm{b}, \mathrm{c}]} / 500$ & 0.5 & 723 \\
\hline $\mathrm{F} 87 \mathrm{~A} / \mathrm{Y} 256 \mathrm{I} / \mathrm{M} 237 \mathrm{I}^{[\mathrm{b}, \mathrm{c}]} / 500$ & 0 & 69 \\
\hline $\mathrm{F} 87 \mathrm{~A} / \mathrm{Y} 256 \mathrm{I} / \mathrm{M} 237 \mathrm{I}^{[\mathrm{b}, \mathrm{c}]} / 500$ & 0.5 & 158 \\
\hline F87A/Y198I/M237I ${ }^{[\mathrm{c}]} / 50$ & 0 & 25 \\
\hline F87A/Y198I/M237I ${ }^{[c]} / 50$ & 2 & $5046 \pm 36$ \\
\hline F87A/Y198I/M237I / 50 & 0 & 12 \\
\hline F87A/Y198I/M237I / 50 & 2 & $7912 \pm 38$ \\
\hline F87A/Y160I/Y198I/M237I / 20 & 0 & 17 \\
\hline F87A/Y160I/Y198I/M237I/ 20 & 2 & $8838 \pm 75$ \\
\hline $\mathrm{F} 87 \mathrm{~A} / \mathrm{Y} 160 \mathrm{I} / \mathrm{Y} 256 \mathrm{I} / \mathrm{M} 237 \mathrm{I} / 50$ & 0 & 45 \\
\hline $\mathrm{F} 87 \mathrm{~A} / \mathrm{Y} 160 \mathrm{I} / \mathrm{Y} 256 \mathrm{I} / \mathrm{M} 237 \mathrm{I} / 50$ & 0.5 & 1605 \\
\hline F87A/Y198I/Y256I/M237I / 50 & 0 & 47 \\
\hline F87A/Y198I/Y256I/M237I / 50 & 0.5 & 1334 \\
\hline F87A/Y160I/Y198I/Y256I/M237I / 50 & 0 & 53 \\
\hline F87A/Y160I/Y198I/Y256I/M237I / 50 & 0.5 & 866 \\
\hline
\end{tabular}

${ }^{[a]}$ Reaction conditions: P450BM3(20-500 nM), $\mathrm{H}_{2} \mathrm{O}_{2}(20 \mathrm{mM})$, Im-C6-Phe (0.5-2 mM), guaiacol $(10 \mathrm{mM})$ in $\mathrm{pH} 7.0$ phosphate buffer at $25^{\circ} \mathrm{C}$; ${ }^{[b]} 4 \mathrm{mM}$ guaiacol is used; ${ }^{[c]} \mathrm{pH}$ 8.0. ${ }^{[\mathrm{d}]} \mathrm{TTN}$ : Total turnover numbers were estimated by the highest absorption peak at $470 \mathrm{~nm}$. 
Table S8 Oxidation of 2,6-dimethoxyphenol catalyzed by the P450BM3 mutants in the absence/presence of Im-C6-Phe ${ }^{[a]}$

\begin{tabular}{|c|c|c|}
\hline Mutants & Im-C6-Phe & $\mathrm{TTN}^{[\mathrm{b}]}$ \\
\hline F87A & - & $28 \pm 2$ \\
\hline F87A & + & $1569 \pm 7$ \\
\hline F87A/Y160I/ Y198I & - & $263 \pm 9$ \\
\hline F87A/Y160I/ Y198I & + & $5100 \pm 48$ \\
\hline F87A/Y160I/ Y256I & - & $157 \pm 1$ \\
\hline F87A/Y160I/ Y256I & + & $3234 \pm 66$ \\
\hline F87A/Y198I/ Y256I & - & $73 \pm 3$ \\
\hline F87A/Y198I/ Y256I & + & $1537 \pm 6$ \\
\hline F87A/Y160I/ Y198I/Y256I & - & $168 \pm 1$ \\
\hline F87A/Y160I/Y198I/Y256I & + & $1670 \pm 8$ \\
\hline F87A/Y160I/ M237I & - & $190 \pm 1$ \\
\hline F87A/Y160I/ M237I & + & $6179 \pm 85$ \\
\hline F87A/Y198I/ M237I & - & $193 \pm 1$ \\
\hline F87A/Y198I/ M237I & + & $6080 \pm 27$ \\
\hline F87A/Y256I/ M237I & - & $131 \pm 0$ \\
\hline F87A/Y256I/ M237I & + & $1101 \pm 22$ \\
\hline F87A/Y160I/ Y198I/M237I & - & $477 \pm 7$ \\
\hline F87A/Y160I/Y198I/ M237I & + & $8453 \pm 170$ \\
\hline F87A/Y160I/Y256I/M237I & - & $166 \pm 1$ \\
\hline F87A/Y160I/Y256I/ M237I & + & $1037 \pm 15$ \\
\hline F87A/Y198I/ Y256I/M237I & - & $139 \pm 4$ \\
\hline F87A/Y198I/Y256I/ M237I & + & $926 \pm 15$ \\
\hline F87A/Y160I/Y198I/ Y256I/M237I & - & $156 \pm 0$ \\
\hline F87A/Y160I/Y198I/Y256I/ M237I & + & $653 \pm 53$ \\
\hline
\end{tabular}

${ }^{[a]}$ Reaction conditions: P450BM3 $(0.02 \mu \mathrm{M})$, Im-C6-Phe (0.5 mM), 2,6-dimethoxyphenol (4 mM), $\mathrm{H}_{2} \mathrm{O}_{2}(20 \mathrm{mM})$ in $\mathrm{pH} 7.0$ phosphate buffer at $25{ }^{\circ} \mathrm{C}$. ${ }^{[b]} \mathrm{TTN}$ : Total turnover numbers were estimated by the highest absorption peak at $468 \mathrm{~nm}$. 
Table S9 Oxidation of $o$-phenylenediamine catalyzed by the P450BM3 mutants in the absence/presence of Im-C6-Phe ${ }^{[a]}$

\begin{tabular}{|c|c|c|}
\hline Mutants & Im-C6-Phe & $\mathrm{TTN}^{[\mathrm{b}]}$ \\
\hline F87A & - & $970 \pm 50$ \\
\hline F87A & + & $19225 \pm 410$ \\
\hline F87A/Y160I/ Y198I & - & $2723 \pm 582$ \\
\hline F87A/Y160I/Y198I & + & $32563 \pm 957$ \\
\hline F87A/Y160I/ Y256I & - & $885 \pm 115$ \\
\hline F87A/Y160I/ Y256I & + & $9792 \pm 433$ \\
\hline F87A/Y198I/ Y256I & - & $788 \pm 77$ \\
\hline F87A/Y198I/ Y256I & + & $6099 \pm 257$ \\
\hline F87A/Y160I/Y198I/Y256I & - & $1003 \pm 40$ \\
\hline F87A/Y160I/Y198I/Y256I & + & $5420 \pm 339$ \\
\hline F87A/Y160I/ M237I & - & $2229 \pm 91$ \\
\hline F87A/Y160I/ M237I & + & $16931 \pm 336$ \\
\hline F87A/Y198I/ M237I & - & $2010 \pm 235$ \\
\hline F87A/Y198I/ M237I & + & $20370 \pm 1005$ \\
\hline F87A/Y256I/ M237I & - & $949 \pm 33$ \\
\hline F87A/Y256I/ M237I & + & $2976 \pm 154$ \\
\hline F87A/Y160I/ Y198I/M237I & - & $3486 \pm 604$ \\
\hline F87A/Y160I/Y198I/ M237I & + & $26076 \pm 2394$ \\
\hline F87A/Y160I/ Y256I/M237I & - & $873 \pm 206$ \\
\hline F87A/Y160I/Y256I/ M237I & + & $2610 \pm 74$ \\
\hline F87A/Y198I/ Y256I/M237I & - & $850 \pm 23$ \\
\hline F87A/Y198I/Y256I/ M237I & + & $2823 \pm 92$ \\
\hline F87A/Y160I/Y198I/ Y256I/M237I & - & $851 \pm 94$ \\
\hline F87A/Y160I/Y198I/Y256I/ M237I & + & $1821 \pm 87$ \\
\hline
\end{tabular}


Table S10 Oxidation of $p$-phenylenediamine catalyzed by the P450BM3 mutants in the absence/presence of Im-C6-Phe ${ }^{[a]}$

\begin{tabular}{|c|c|c|c|c|}
\hline & Im-C6-Phe & A500 & $\begin{array}{c}\mathrm{A} 500^{-} \\
\mathrm{A} 500_{\text {blank }} \text { [c] }\end{array}$ & $\operatorname{TTN}^{[\mathrm{d}]}$ \\
\hline $\mathrm{PPD}+\mathrm{H}_{2} \mathrm{O}_{2}[\mathrm{~b}]$ & - & $0.157 \pm 0.002$ & - & - \\
\hline F87A & - & $0.179 \pm 0.006$ & $0.022 \pm 0.006$ & $804 \pm 216$ \\
\hline F87A & + & $0.406 \pm 0.019$ & $0.249 \pm 0.019$ & $8981 \pm 674$ \\
\hline F87A/Y160I/Y198I & - & $0.230 \pm 0.018$ & $0.074 \pm 0.018$ & $2658 \pm 664$ \\
\hline F87A/Y160I/Y198I & + & $0.639 \pm 0.001$ & $0.483 \pm 0.001$ & $17408 \pm 49$ \\
\hline F87A/Y160I/Y256I & - & $0.237 \pm 0.012$ & $0.080 \pm 0.012$ & $2882 \pm 418$ \\
\hline F87A/Y160I/Y256I & + & $0.358 \pm 0.007$ & $0.201 \pm 0.007$ & $7248 \pm 240$ \\
\hline F87A/Y198I/Y256I & - & $0.193 \pm 0.006$ & $0.036 \pm 0.006$ & $1307 \pm 215$ \\
\hline F87A/Y198I/Y256I & + & $0.283 \pm 0.020$ & $0.127 \pm 0.020$ & $4563 \pm 729$ \\
\hline F87A/Y160I/Y198I/Y256I & - & $0.219 \pm 0.004$ & $0.062 \pm 0.004$ & $2233 \pm 130$ \\
\hline F87A/Y160I/Y198I/Y256I & + & $0.255 \pm 0.015$ & $0.098 \pm 0.015$ & $3542 \pm 537$ \\
\hline F87A/Y160I/M237I & - & $0.2 \pm 0.008$ & $0.043 \pm 0.008$ & $1556 \pm 283$ \\
\hline F87A/Y160I/M237I & + & $0.505 \pm 0.002$ & $0.348 \pm 0.002$ & $12546 \pm 56$ \\
\hline F87A/Y198I/M237I & - & $0.211 \pm 0.002$ & $0.055 \pm 0.002$ & $1971 \pm 85$ \\
\hline F87A/Y198I/M237I & + & $0.390 \pm 0.018$ & $0.234 \pm 0.018$ & $8429 \pm 664$ \\
\hline F87A/Y256I/M237I & - & $0.201 \pm 0.015$ & $0.044 \pm 0.015$ & $1598 \pm 541$ \\
\hline F87A/Y256I/M237I & + & $0.231 \pm 0.015$ & $0.075 \pm 0.015$ & $2691 \pm 552$ \\
\hline F87A/Y160I/Y198I/M237I & - & $0.203 \pm 0.009$ & $0.047 \pm 0.009$ & $1684 \pm 339$ \\
\hline F87A/Y160I/Y198I/M237I & + & $0.479 \pm 0.022$ & $0.322 \pm 0.022$ & $11612 \pm 781$ \\
\hline F87A/Y160I/Y256I/M237I & - & $0.224 \pm 0.013$ & $0.067 \pm 0.013$ & $2422 \pm 463$ \\
\hline F87A/Y160I/Y256I/M237I & + & $0.251 \pm 0.011$ & $0.094 \pm 0.011$ & $3389 \pm 384$ \\
\hline F87A/Y198I/Y256I/M237I & - & $0.210 \pm 0.013$ & $0.053 \pm 0.013$ & $1906 \pm 453$ \\
\hline F87A/Y198I/Y256I/M237I & + & $0.249 \pm 0.015$ & $0.092 \pm 0.015$ & $3327 \pm 539$ \\
\hline F87A/Y160I/Y198I/Y256I/M237I & - & $0.225 \pm 0.015$ & $0.069 \pm 0.015$ & $2474 \pm 552$ \\
\hline F87A/Y160I/Y198I/Y256I/M237I & + & $0.238 \pm 0.014$ & $0.082 \pm 0.014$ & $2947 \pm 519$ \\
\hline
\end{tabular}

[a] Reaction conditions: P450BM3 $(0.01 \mu \mathrm{M}), \mathrm{H}_{2} \mathrm{O}_{2}(20 \mathrm{mM})$, Im-C6-Phe $(0.5 \mathrm{mM}), p$ phenylenediamine $(4 \mathrm{mM}), \mathrm{H}_{2} \mathrm{O}_{2}(20 \mathrm{mM})$ for 1 hour in $\mathrm{pH} 7.0$ phosphate buffer at $25^{\circ} \mathrm{C}$. ${ }^{[b]}$ The blank control reaction that only contains $4 \mathrm{mM} p$-phenylenediamine, $20 \mathrm{mM} \mathrm{H}_{2} \mathrm{O}_{2}$ for 1 hour in pH 7.0 phosphate buffer at $25^{\circ} \mathrm{C}$. ${ }^{[c]} \mathrm{A} 500-\mathrm{A} 500_{\text {blank: }}$ : Difference of absorption intensity calculated by subtracting the blank control experiment from the observed absorption intensity at $500 \mathrm{~nm}$. ${ }^{\text {d] }}$ TTN: Total turnover numbers were estimated by the difference of absorption intensity calculated. 
Table S11 Observed heme decay rate in pseudo-first order reactions of P450BM3 variants with $\mathrm{H}_{2} \mathrm{O}_{2}$ in the absence/presence of Im-C6-Phe and styrene. ${ }^{\mathrm{a}}$

\begin{tabular}{lcccc}
\hline P450BM3 variants & \multicolumn{3}{c}{$k_{\text {obs }}{ }^{\mathrm{b}}$} \\
\cline { 2 - 5 } & $\mathrm{H}_{2} \mathrm{O}_{2}{ }^{\mathrm{c}}$ & Styrene $^{\mathrm{d}}$ & Im-C6-Phe $^{\mathrm{e}}$ & Im-C6-Phe/styrene $^{\mathrm{f}}$ \\
\hline $\mathrm{F}_{87 \mathrm{~A}^{\mathrm{g}}}$ & $4.7 \times 10^{-4}$ & - & $5.1 \times 10^{-3}$ & $1.9 \times 10^{-3}$ \\
F87A/Y160I/Y198I & $5.7 \times 10^{-3}$ & $7.2 \times 10^{-4}$ & $2.5 \times 10^{-2}$ & $6.9 \times 10^{-3}$ \\
F87A/Y198I/M237I & $8.0 \times 10^{-3}$ & $9.3 \times 10^{-4}$ & $2.4 \times 10^{-2}$ & $5.8 \times 10^{-3}$ \\
F87A/Y160I/Y198I/M237I & $2.1 \times 10^{-2}$ & $1.6 \times 10^{-3}$ & $4.1 \times 10^{-2}$ & $1.2 \times 10^{-2}$ \\
\hline
\end{tabular}

${ }^{a}$ Conditions: P450BM3 variants $(2 \mu \mathrm{M}), \mathrm{H}_{2} \mathrm{O}_{2}(20 \mathrm{mM})$, Im-C6-Phe $(0.5 \mathrm{mM}$, if added), styrene (4 mM, if added); ${ }^{\mathrm{b}} k_{\text {obs }}$ is the observed heme decay rate; ${ }^{\mathrm{c}}$ Only $\mathrm{H}_{2} \mathrm{O}_{2}$ was added; ${ }^{\mathrm{d} S t y r e n e}$ was added; ${ }^{\mathrm{e}} \mathrm{Im}$-C6-Phe was added but without styrene; ${ }^{\mathrm{f}}$ Both Im-C6-Phe and styrene was added; $\mathrm{g}$ Ref. 1. 